Bangladesh J. Plant Taxon. 28(1): 97-124, 2021 (June)

(C) 2021 Bangladesh Association of Plant Taxonomists

https://doi.org/10.3329/bjpt.v28i1.54211

\title{
FLORISTIC COMPOSITION OF THE COASTAL DISTRICT SATKHIRA, BANGLADESH
}

\author{
Gazi Mosharof Hossain ${ }^{1}$, Saleh Ahammad Khan, Md. Abdur Rahim, \\ Mohammad Sayedur Rahman ${ }^{2}$ and Khandaker Mohammad Noor Islam \\ Plant Systematics and Biodiversity Laboratory, Department of Botany, \\ Jahangirnagar University, Savar, Dhaka, Bangladesh
}

Keywords: Flora; Angiosperms; Satkhira; Bangladesh.

\begin{abstract}
This study presents elementary taxonomic data on the composition of vascular flora in the disaster prone coastal district Satkhira of Bangladesh. The results of this study show that this district houses 664 species under 468 genera and 133 families of vascular plants. The Pteridophytes and Gymnosperms are represented by 21 and five species, whereas, the Magnoliopsida (dicotyledons) and Liliopsida (monocotyledons) of Angiosperms by 494 and 144 species, respectively. About 59.64\% of these species are herbs, $15.96 \%$ shrubs, $21.69 \%$ trees, $2.26 \%$ palms and $0.45 \%$ bamboos. Total $73.04 \%$ of the species are wild, $19.88 \%$ planted and $7.08 \%$ cultivated. In this area, Fabaceae with 31 species is the largest family, followed by Asteraceae with 27 species, Apocynaceae with 26 species and Malvaceae with 25 and Acanthaceae with 22 species. Ficus with seven species is recorded as the largest genus, which is followed by Solanum, Hygrophila, Euphorbia, Senna, Phyllanthus, Ipomoea, Hibiscus and Alternanthera. Most of the species are found in fallow lands, roadsides, woodlands, homesteads and scrub jungles and useful as medicine, ornamental, fibre, fruit, livestock feed, soil binder and timber. The floristic composition of this district is still rich, though most of its habitats and ecosystems are vulnerable to natural disasters. Effective conservation plan and measures should be adopted for the precious flora of this coastal district.
\end{abstract}

\section{Introdfuction}

Knowledge on floristic composition and structure is essential in identifying the important elements and understanding the status, extent, assessment of plant biodiversity (WCMC, 1992). It is also useful in exploration of alternative species, identification and conservation of threatened species and helpful in sustainable utilization of plant biodiversity. Thus, studies on floristic composition and structure of different areas and countries throughout the world become imperative due to which large-scale floristic inventories have been conducted in various forestand non-forest areas globally for over a century (Ostertag et al., 2014).

As a significant part of the South Asian Mega Centre of genetic diversity (Chowdhury, 1996), Bangladesh houses a rich biodiversity including a huge number of plant species (Approx. 5000 species of Angiosperms, Khan, 1977), though it is one of the countries, most vulnerable to climate change, and ranked as one of the world's most disaster-prone area (Choudhury, 2002; World Bank, 2005). However, the floristic composition of this country has so far been partially explored through various kinds of inventories conducted in 18th to 20th centuries. The large-scale floristic studies covering the area of the present political boundary of Bangladesh does do not provide habitat-, area- or district-specific detail data on floristic composition (Hooker, 1872-1897; Prain,

\footnotetext{
${ }^{1}$ Corresponding author. Email: gazibotju@gmail.com

${ }^{2}$ Bangladesh National Herbarium, Chiriakhana Road, Mirpur 1, Dhaka 1216, Bangladesh
} 
1903; Siddiqui et al., 2007; Ahmed et al., 2008-2009; Ahmed et al., 2009). Most of the small to medium scale floristic studies conducted in this country so far cover mostly the north-eastern and south-eastern highland regions and forested areas of this country (Uddin et al., 1998; Khan and Huq, 2001; Rashid and Mia, 2001; Uddin et al., 2003; Islam et al., 2009; Arefin et al., 2011; Rahman, 2017; Haque et al., 2018; Uddin and Hassan, 2018). Some studies in plain land and coastal regions of the country have also been conducted (Sultana, 2012; Rahman et al., 2015; Tabassum, 2015; Shetu et al., 2018; Rahman et al., 2019; Khanam et al., 2020; Roy and Khan, 2020). In spite of these studies, the floristic composition in most of the areas of this country is unknown till now. Therefore, conducting adequate studies on the floristic composition in these areas are crucial to know the composition, structure and status of their plant resources.

Satkhira is one of the costal districts of Bangladesh that are most vulnerable to the natural disasters, which affect the coastal region frequently, and sometimes severely. This district is one of the most risk prone areas of Bangladesh, because most of its habitats and ecosystems are being degraded due to the consequences of different natural disasters and human interventions, and most of its natural vegetation are being replaced by settlements, agricultural expansions, shrimp cultures and other human activities. Protecting and maintaining the biodiversity in the coastal areas is one of the biggest conservation challenges today. However, the first key step to achieve this goal is to complete the floristic explorations and compile the updated lists of species extant in these areas (Schaminée et al., 2011; Sharrock, 2012). But so far, no floristic study is known to be carried out throughout Satkhira district based on field inventories and examination of plant specimens. The floristic study of Rahman et al. (2015) covers the Sundarban Mangrove area of this district belonging to its Shamnagar upazila but it does not provide specific information on plant species composition and distribution in this administrative area.

Floristic studies throughout this district are required to generate the baseline taxonomic data on the current composition and status of plant species, help in adopting appropriate conservation initiatives for the threatened or near threatened species, promote the plant resource-based socioeconomic development projects and contribute in future studies on the change in floristic composition, plant species diversity and vegetation in this area. Therefore, there is a great scope and need for conducting a detail floristic study throughout this district. This study has been carried out to construct a taxonomic checklist and provide quantitative data on the vascular plants of Satkhira district based on thorough taxonomic inventories throughout the area.

\section{Materials and Methods}

Satkhira district, located in between $21^{\circ} 36^{\prime}-22^{\circ} 54^{\prime} \mathrm{N}$ and $88^{\circ} 54^{\prime}-89^{\circ} 20^{\prime} \mathrm{E}$, comprises an area of $3,817.29 \mathrm{~km}^{2}$, including $1632.00 \mathrm{~km}^{2}$ forests, and consists of seven upazilas and 79 unions (Population and Housing Census, 2015). It is bounded by Jessore district on the north, the Bay of Bengal on the south, Khulna district on the east, West Bengal state of India on the west. The topography of this district is flat, with a maximum elevation range of 17.06 metre and an average elevation above sea level of 6.40 metre. This area is covered by cropland, grassland, wet lands, scrub jungles, mangrove forests and homestead gardens etc. Over the course of the year, the temperature typically varies from $13.89^{\circ} \mathrm{c}$ to $35^{\circ} \mathrm{c}$ and is rarely below $11^{\circ} \mathrm{C}$ or above $37.78^{\circ} \mathrm{C}$. Since the last decade, the trend of change in maximum, minimum and mean temperature in rainy and summer seasons in Satkhira district is increasing, whereas that in minimum and mean temperature in winter season and total rainfall in rainy and summer seasons are decreasing (http://datalibrary.bmd.gov.bd). In the muggiest months, the highest humidity ranges from $73 \%$ to 92\% (www.world weatheronline.com). 
This study was based on a thorough taxonomic inventory conducted in different seasons of 2017-2020 throughout the Satkhira district. The collection, processing, drying and preservation of plant specimens were done following standard herbarium methods and techniques (Bridson and Forman, 1989; Singh and Subramaniam, 2008). The representative plant specimens of all taxa were examined at Plant Systematics and Biodiversity Laboratory of Jahangirnagar University and Bangladesh National Herbarium (DACB). Identification of the specimens was completed through consulting the taxonomic descriptions and keys available in the relevant literatures (Hooker, 18721897; Prain, 1903; Wu and Raven, 1994-2001; Wu et al., 1999-2013; Siddiqui et al., 2007; Ahmed et al., 2008-2009; Ahmed et al., 2009), and by matching with the respective voucher specimens of DACB and Jahangirnagar University Herbarium (JUH). The relevant type images available in the web pages of different international herbaria, such the Royal Botanic Gardens Kew (K), and the Conservatoire et Jardin botaniques de la Ville de Genève (G) etc., and illustration of Flora of China (Wu and Raven, 1994-2001; Wu et al., 1999-2013) were also matched. All voucher specimens have been preserved at JUH.

Nomenclatural information were verified following Flora of China (Wu and Raven, 19942001; Wu et al., 1999-2013), The Plant List (2013) and TROPICOS (2017). The bangla names were collected from Huq (2019), Siddiqui et al. (2007), Ahmed et al. (2008-2009) and Ahmed et al. (2009), and through interviews with the local people. The families of Pteridophytes and Gymnosperms have been arranged following Pichi (1977) and Kramer and Green (1990), respectively, and those of Angiosperms following Cronquist (1981), whereas the genera and species under each family alphabetically (Table 1 ).

Data on uses of the species were collected through interviews with the local people during the field surveys, and consulting the relevant literatures (Ghani, 1998; Van Valkenburg and Bunyapraphatsara, 2002; Siddiqui et al., 2007; Ahmed et al., 2008-2009; Ahmed et al., 2009). The status of rare and threatened plant species in the study area was inferred through field observation and estimation on population size, distribution range and regeneration of each species in the area.

\section{Results and Discussion}

During this study the vascular flora of Satkhira district was found to be composed of 664 species under 468 genera and 133 families. Pteridophytes were represented by 21 species of 18 genera under 12 families and Gymnosperms by five species of four genera under four families, and Magnoliopsida (dicotyledons) and Liliopsida (monocotyledons) of Angiosperms by 494 and 144 species of 348 and 98 genera under 92 and 25 families, respectively (Table 1 and Fig. 1). Total $396(59.64 \%)$ of these species were herbs, $144(21.69 \%)$ trees, $106(15.96 \%)$ shrubs, 15 (2.26\%) palms and three (0.45\%) bamboos. Most of the herbs were erect (48.99\%), climber (19.19

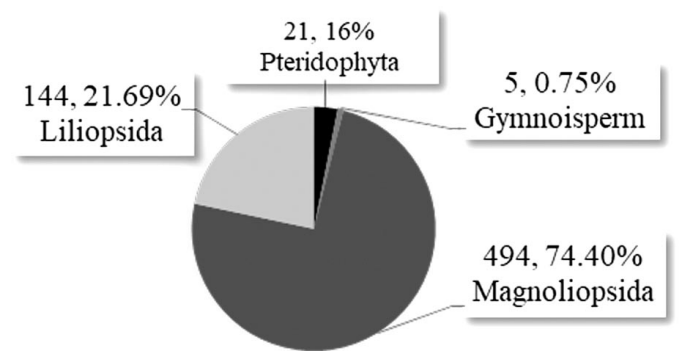

Fig. 1. Species composition (number and percent of species) of different plant groups of Satkhira district. 
$\%)$ and prostrate $(16.92 \%)$ that were followed by creeper, parasite, free floating, submerged and epiphyte (Fig. 2). All species of Pteridophytes were found as wild but those of the Gymnosperms as planted, whereas among the Angiosperms total 464 (72.73\%) species were wild, 127 (19.91\%) planted and $47(7.37 \%)$ cultivated. $349(75.22 \%)$ species of the dicots and $115(24.78 \%)$ species of the monocots were wild and the rest were cultivated or planted.

In Magnoliopsida, Fabaceae with 31 species under 19 genera, representing $4.72 \%$ of the vascular flora of Satkhira district, was the largest family, followed by Asteraceae with 27 species of 26 genera, Apocynaceae with 26 species of 23 genera, Malvaceae with 25 species of 17 genera, Acanthaceae with 22 species of 13 genera, Rubiaceae with 18 species under 12 genera, Cucurbitaceae and Lamiaceae, each with 17 species under 12 and 14 genera, respectively, Solanaceae with 15 species of eight genera, Caesalpiniaceae with 13 species of eight genera, Moraceae with 12 species under four genera, and Amaranthaceae and Mimosaceae, each with 12 species under eight genera. Ficus with seven species was recorded as the largest genus, which was followed by Euphorbia, Hygrophila, Solanum, each with six species, Hibiscus, Ipomoea, Phyllanthus and Senna, each with five species, and Alternanthera, Amaranthus, Dalbergia, Desmodium, Ludwigia, Oldenlandia, Persicaria, Sida, Terminalia and Vigna, each with four species.

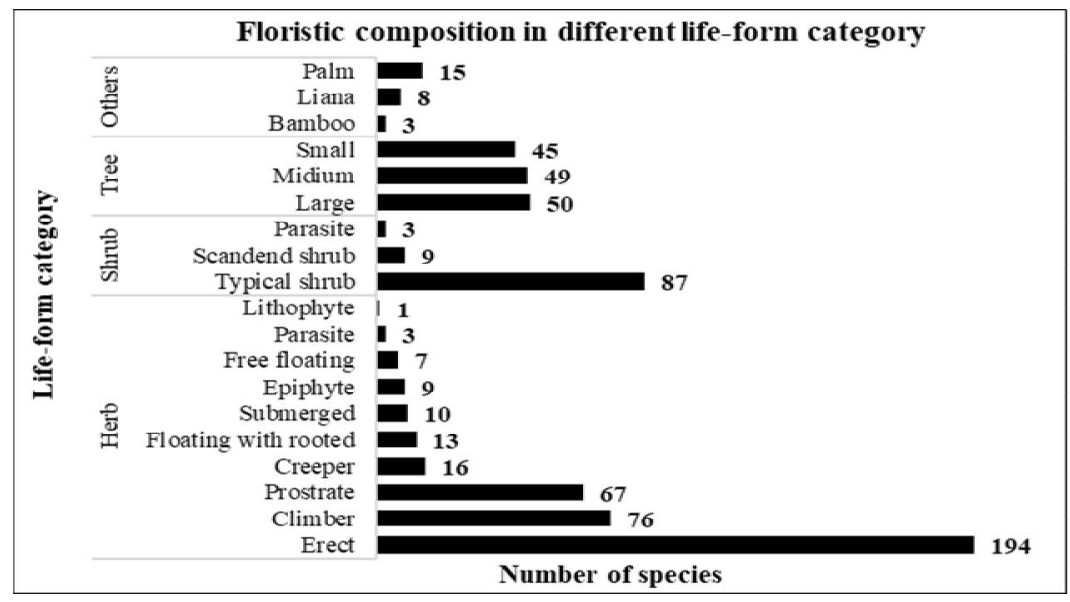

Fig. 2. Floristic composition in different life-form categories of Satkhira district.

In Liliopsida, Poaceae with 44 species under 30 genera, comprising $6.70 \%$ of the vascular flora of this area, was the largest family, and followed by Cyperaceae with 22 species under eight genera, Arecaceae and Araceae, each with 15 species uder 12 and 13 genera, respectively. Cyperus with 10 species was the largest genus, which was followed by Commelina and Paspalum, each with four species and Bambusa and Calamus, each with three species.

In Satkhira district, total 403 (60.69\%) plant species were found to be distributed in Satkhira sadar, 355 (53.46\%) in Shaymnagar, 334 (50.30\%) in Kaligonj, 318 (47.89\%) in Debhata, 315 (47.44\%) in Kolarowa, 296 (44.58\%) in Tala and 235 (35.39\%) in Ashasuni upazila, but most of the species found in each of these upazilas were overlapping with that of other upazila/s. A total of $152(22.89 \%)$ species were found to be distributed in a part of the Sundarbans mangrove forest, situated in the southern part of Shaymnagar upazila of Satkhira district, of which 65 (9.79\%) species were exclusively distributed in that mangrove habitat and the rest $87(13.10 \%)$ species were found to overlapping with other upazilas of this study area. 
Table 1. List of vascular plant species of Satkhira district, Bangladesh.

\begin{tabular}{|c|c|c|c|c|c|c|}
\hline Scientific name & Bangla name & Habit & Habitat & Distribution & Use & RSE \\
\hline \multicolumn{7}{|l|}{$\begin{array}{l}\text { PTERIDOPHYTA Schimp. } \\
\text { Selaginellaceae Willk }\end{array}$} \\
\hline Selaginella vaginata Spring & Selaginella & Herb, pr; w & $\mathrm{Ml}, \mathrm{Wl}$ & $\mathrm{De}, \mathrm{Ka}, \mathrm{Sh}^{+}$ & M & GMH 5207 \\
\hline \multicolumn{7}{|l|}{ Ophioglossaceae Martinov } \\
\hline \multicolumn{6}{|l|}{ Salviniaceae Martinov } & GMH 5202 \\
\hline Azolla pinnata $\mathrm{R}$. Br. & Khudipana & Herb, fl; w & Wtl & As, Ka, Ss, Ta & $\mathrm{Gm}$ & GMH 5205 \\
\hline Salvinia adnata Desv. & Pani dhekia & Herb, fl; w & Wtl & As, De, Ta & $\mathrm{Gm}, \mathrm{O}$ & GMH 5200 \\
\hline S. cucullata Roxb. & Indur kani & Herb, fl; w & Wtl & $\mathrm{Ka}, \mathrm{Ss}, \mathrm{Sh}^{+}, \mathrm{Ta}$ & $\mathrm{Gm}, \mathrm{O}$ & GMH 5211 \\
\hline \multicolumn{7}{|l|}{ Marsileaceae Mirb. } \\
\hline $\begin{array}{l}\text { Marsilea quadrifolia L. } \\
\text { Lygodiaceae M. Roem. }\end{array}$ & Susni shak & Herb, cr; w & Af, Fl, Wtl & All upazilas ${ }^{+}$ & $\mathrm{Vg}$ & GMH 5204 \\
\hline $\begin{array}{l}\text { Lygodium flexuosum (L.) Sw. } \\
\text { Pteridaceae E.D.M. Kirchn. }\end{array}$ & Saralata fern & Herb, cl; w & $\mathrm{Sj}, \mathrm{Wl}$ & $\mathrm{De}, \mathrm{Ka}, \mathrm{Ko}$ & M & GMH 5201 \\
\hline *Acrostichum aureum $\mathrm{L}$. & Hudo & Herb, er; w & Fm, Wl & $\mathrm{Sh}^{+}$ & $\mathrm{M}, \mathrm{Tm}$ & GMH 5208 \\
\hline Adiantum caudatum $\mathrm{L}$. & Khopa fern & Herb, ep; w & Op, Obw & $\mathrm{De}, \mathrm{Ka}, \mathrm{Ko}$ & $\mathrm{O}$ & MAR 2901 \\
\hline A. tenerum $\mathrm{Sw}$. & Biddapata & Herb, ep; w & Op, Obw & $\mathrm{De}, \mathrm{Ka}$ & $\mathrm{O}$ & MAR 2902 \\
\hline $\begin{array}{l}\text { Ceratopteris pteridoides (Hook.) } \\
\text { Hieron. }\end{array}$ & Pani dhekia & Herb, er; w & Wtl & $\mathrm{Sh}^{+}$ & $\mathrm{Vg}$ & MSR 3321 \\
\hline C. thalictroides (L.) Brongn. & Pani lettuce & Herb, er; w & Wtl & De, Sh, Ss, Ta & $\mathrm{Vg}$ & MSR 3310 \\
\hline \multicolumn{7}{|l|}{ Vittariaceae Ching } \\
\hline $\begin{array}{l}\text { *Haplopteris elongate (Sw.) E.H. } \\
\text { Crane }\end{array}$ & Viteria & Herb, ep; w & Op, Wl & $\mathrm{Sh}^{+}$ & $\mathrm{M}, \mathrm{O}$ & MSR3 302 \\
\hline \multicolumn{7}{|l|}{ Polypodiaceae J. Presl \& C. Presl } \\
\hline Drynaria quercifolia (L.) J. Sm. & Pankhiraj & Herb, ep; w & Op, Wl & All upazilas ${ }^{+}$ & $\mathrm{M}, \mathrm{O}$ & GMH 5210 \\
\hline Microsorum punctatum (L.) Copel. & Gucha patra & Herb, ep; w & Op, Wl & $\mathrm{De}, \mathrm{Ka}, \mathrm{Sh}^{+}$ & $\mathrm{M}, \mathrm{O}$ & GMH 5206 \\
\hline Pyrrosia nuda (Giesenh.) Ching & Pyrosia & Herb, ep; w & Op, Wl & $\mathrm{De}, \mathrm{Ka}, \mathrm{Sh}^{+}$ & M & GMH 5212 \\
\hline \multicolumn{7}{|l|}{ Blechnaceae Newman } \\
\hline $\begin{array}{l}\text { Stenochlaena palustris (Burm. f.) } \\
\text { Bed. }\end{array}$ & Dhekia lata & Herb, cl; w & $\mathrm{Sj}, \mathrm{Wl}$ & $\mathrm{Ka}, \mathrm{Ss}, \mathrm{Ta}$ & $\mathrm{M}, \mathrm{Vg}$ & GMH 5209 \\
\hline \multicolumn{7}{|l|}{$\begin{array}{l}\text { Thelypteridaceae Ching ex Pic. } \\
\text { Serm. }\end{array}$} \\
\hline $\begin{array}{l}\text { Ampelopteris prolifera (Retz.) } \\
\text { Copel. }\end{array}$ & Dheki shak & Herb, cr; w & $\mathrm{Fl}, \mathrm{Fm}, \mathrm{Wl}$ & All upazilas & M & MAR 2903 \\
\hline $\begin{array}{l}\text { Thelypteris dentata (Forssk.) E.P. } \\
\text { St.John }\end{array}$ & Datitila & Herb, cr; w & $\mathrm{Fl}, \mathrm{Fm}, \mathrm{Wl}$ & $\mathrm{De}, \mathrm{Ka}$ & $\mathrm{O}, \mathrm{Vg}$ & MAR 2904 \\
\hline \multicolumn{7}{|l|}{ Athyriaceae Alston } \\
\hline \multicolumn{6}{|l|}{ GYMNOSPERMS Prantl } & MAR 2905 \\
\hline \multicolumn{7}{|l|}{ Cycadaceae Pers. } \\
\hline Cycas circinalis $\mathrm{L}$. & Cycas & Tree, s; pl & $\mathrm{Hs}$ & Ss & $\mathrm{M}, \mathrm{O}$ & MAR 2907 \\
\hline C. revoluta Thunb. & Moniraj & Tree, s; pl & $\mathrm{Hs}$ & Ss & M, O & MAR 2908 \\
\hline \multicolumn{7}{|l|}{$\begin{array}{l}\text { Araucariaceae Henkel \& W. } \\
\text { Hochst. }\end{array}$} \\
\hline $\begin{array}{l}\text { Araucaria heterophylla (Salisb.) } \\
\text { Franco }\end{array}$ & $\mathrm{X}$-mas tree & Tree, m; pl & $\mathrm{Hs}$ & $\mathrm{De}, \mathrm{Ka}, \mathrm{Ss}$ & $\mathrm{O}$ & MAR 2906 \\
\hline
\end{tabular}


Table 1 Contd.

\begin{tabular}{|c|c|c|c|c|c|c|}
\hline Scientific name & Bangla name & Habit & Habitat & Distribution & Use & RSE \\
\hline \multicolumn{7}{|l|}{ Cupressaceae Gray } \\
\hline $\begin{array}{l}\text { Thuja plicata Donn ex D. Don } \\
\text { Pinaceae Spreng. ex Rudolphi }\end{array}$ & Thuja & Shrub; pl & Hs, Ml & As, De, Ka, Ss & $\mathrm{O}$ & GMH 5219 \\
\hline $\begin{array}{l}\text { Pinus caribaea Morelet } \\
\text { MAGNOLIOPSIDA Brongn. } \\
\text { Magnoliaceae Juss. }\end{array}$ & Pine gach & Tree, $1 ; \mathrm{pl}$ & Hs, Rs & Ss & $\mathrm{O}$ & MAR 2909 \\
\hline $\begin{array}{l}\text { Magnolia champaca (L.) Baill. ex } \\
\text { Pierre }\end{array}$ & Champa & Tree, $1 ; \mathrm{pl}$ & Rs & Ss & $\mathrm{M}, \mathrm{O}$ & $S A K 2001$ \\
\hline \multicolumn{7}{|l|}{ Annonaceae Juss. } \\
\hline Annona reticulata $\mathrm{L}$. & Atta & Tree, s; w & $\mathrm{Hs}, \mathrm{Sj}$ & All upazilas & $\mathrm{Fr}$ & $S A K 2102$ \\
\hline A. squamosa $\mathrm{L}$. & Shorifa & Tree, s; pl & Hs & $\mathrm{De}, \mathrm{Ka}, \mathrm{Ss}$ & $\mathrm{Fr}$ & SAK 2103 \\
\hline $\begin{array}{l}\text { Artabotrys hexapetalus (L. f.) } \\
\text { Bhandari }\end{array}$ & Kathali chapma & Shrub, sc; pl & Hs & $\mathrm{Ka}, \mathrm{De}, \mathrm{Ss}$ & $\mathrm{M}, \mathrm{O}$ & SAK 2104 \\
\hline $\begin{array}{l}\text { Huberantha pendula (Capuron ex G. } \\
\text { E. Schatz \& Le Thomas) Chaowasku }\end{array}$ & Weeping debdaru & Tree, $\mathrm{m} ; \mathrm{pl}$ & Rs & $\mathrm{De}, \mathrm{Ko}, \mathrm{Ss}$ & $\mathrm{O}$ & SAK 2106 \\
\hline $\begin{array}{l}\text { Polyalthia longifolia (Sonn.) } \\
\text { Thwaites }\end{array}$ & Debdaru & Tree, $1 ; \mathrm{Pl}$ & Rs, Wl & All upazilas ${ }^{+}$ & $\mathrm{O}, \mathrm{T}$ & SAK 2105 \\
\hline $\begin{array}{l}\text { P. suberosa (Roxb.) Thwaites } \\
\text { Lauraceae Juss. }\end{array}$ & Barachali & Tree, s; w & $\mathrm{Fl}, \mathrm{Wl}$ & De, Ka, Ko & $\mathrm{Fr}, \mathrm{Fw}$ & SAK 2107 \\
\hline $\begin{array}{l}\text { Cinnamomum tamala (Buch.- } \\
\text { Ham.) T. Nees \& Eberm. }\end{array}$ & Tejpata & Tree, $\mathrm{m} ; \mathrm{pl}$ & Hs & $\mathrm{Ka}, \mathrm{Ss}$ & $\mathrm{Sp}$ & $S A K 2108$ \\
\hline C. verum J. Presl & Darchini & Tree, $\mathrm{m} ; \mathrm{pl}$ & $\mathrm{Hs}$ & Ko, Ss & $\mathrm{Sp}$ & $S A K 2109$ \\
\hline Litsea glutinosa (Lour.) C.B. Rob. & Kukurchita & Tree, $\mathrm{m} ; \mathrm{w}$ & $\mathrm{Sj}, \mathrm{Wl}$ & $\mathrm{De}, \mathrm{Ka}, \mathrm{Ko}$ & M & $S A K 2110$ \\
\hline $\begin{array}{l}\text { L. monopetala (Roxb.) Pers. } \\
\text { Piperaceae Giseke }\end{array}$ & Boro-kukurchita & Tree, m; w & $\mathrm{Sj}, \mathrm{Wl}$ & $\mathrm{De}, \mathrm{Ka}$ & M & $S A K 2111$ \\
\hline Peperomia pellucida (L.) Kunth & Luchi pata & Herb, pr; w & $\mathrm{Gl}, \mathrm{Sj}$ & All upazilas ${ }^{+}$ & M & MAR 2910 \\
\hline Piper betle L. & Pan & Herb, cl; cv & Af & $\mathrm{De}, \mathrm{Ka}$ & M & MAR 2911 \\
\hline P. longum $\mathrm{L}$. & Pipul & Herb, cl; w & $\mathrm{Fl}, \mathrm{Wl}$ & $\mathrm{De}, \mathrm{Ka}, \mathrm{Ko}$ & M & MAR 2912 \\
\hline P. retrofractum Vahl & Choi jhal & Herb, cl; pl & Hs, Wl & $\mathrm{De}, \mathrm{Ka}$ & $\mathrm{M}, \mathrm{Sp}$ & MAR 2913 \\
\hline $\begin{array}{l}\text { Aristolochiaceae Juss. } \\
\text { Aristolochia indica L. } \\
\text { Nymphaeaceae Salisb. }\end{array}$ & Ishwarmul & Herb, cl; w & $\mathrm{Sj}$ & $\mathrm{Ko}, \mathrm{Ka}, \mathrm{Ss}$ & M & MAR 2914 \\
\hline Nymphaea nouchali Burm. f. & Nil shapla & Herb, fr; w & Wtl & $\mathrm{De}, \mathrm{Sh}, \mathrm{Ss}, \mathrm{Ta}$ & $\mathrm{M}, \mathrm{O}$ & GMH 5213 \\
\hline N. pubescens Willd & Sada shapla & Herb, fr; w & Wtl & As, Sh, Ss, Ta & $\mathrm{O}, \mathrm{Vg}$ & GMH 5214 \\
\hline $\begin{array}{l}\text { N. rubra Roxb. ex Andrews } \\
\text { Ceratophyllaceae Gray }\end{array}$ & Lal shapla & Herb, fr; w & Wtl & $\mathrm{As}, \mathrm{Sh}^{+}, \mathrm{Ss}, \mathrm{Ta}$ & $\mathrm{M}, \mathrm{O}$ & GMH 5215 \\
\hline $\begin{array}{l}\text { Ceratophyllum demersum L. } \\
\text { Ranunculaceae Juss. }\end{array}$ & Kantajhanjhi & Herb, sm; w & Wtl & As, Ss, Sh, Ta & M & MAR 2915 \\
\hline Clematis zeylanica Poir. & Irula & Shrub, li; w & $\mathrm{Sj}$ & $\mathrm{De}$ & M & MAR 2916 \\
\hline $\begin{array}{l}\text { Ranunculus sceleratus L. } \\
\text { Menispermaceae Juss. }\end{array}$ & Jhumka phul & Herb, er; w & Wtl & Sh, Ss, Ta & M & MAR 2917 \\
\hline Cocculus hirsutus (L.) W. Theob. & Huyer & Herb, cl; w & $\mathrm{Sj}$ & $\mathrm{De}, \mathrm{Ka}, \mathrm{Ko}, \mathrm{Ss}$ & M & GMH 5222 \\
\hline Stephania japonica (Thunb.) Miers & Akandi manik & Herb, cl; w & $\mathrm{Sj}, \mathrm{Wl}$ & $\mathrm{De}, \mathrm{Ka}, \mathrm{Ss}$ & M & GMH 5216 \\
\hline Tinospora sinensis (Lour.) Merr. & Gulancha & Herb, cl; w & $\mathrm{Wl}$ & $\mathrm{De}, \mathrm{Ka}, \mathrm{Ss}$ & M & GMH 5218 \\
\hline $\begin{array}{l}\text { Tiliacora racemosa Colebr. } \\
\text { Papaveraceae Juss. }\end{array}$ & Bagh lata & Shrub, li; w & $\mathrm{Sj}, \mathrm{Wl}$ & $\mathrm{De}, \mathrm{Ka}, \mathrm{Ko}, \mathrm{Ss}$ & $\mathrm{M}, \mathrm{Tm}$ & GMH 5221 \\
\hline $\begin{array}{l}\text { Argemone mexicana L. } \\
\text { Cannabaceae Martinov }\end{array}$ & Sheyal kanta & Herb, er; w & Af, Fl, Rs & As, Ko, Ss, Ta & M & MAR 2918 \\
\hline Trema orientalis (L.) Blume & Banjiga & Tree, m; w & $\mathrm{Sj}, \mathrm{Wl}$ & All upazilas ${ }^{+}$ & $\mathrm{Fw}$ & KMNI 001 \\
\hline
\end{tabular}


Table 1 Contd.

\begin{tabular}{|c|c|c|c|c|c|c|}
\hline Scientific name & Bangla name & Habit & Habitat & Distribution & Use & RSE \\
\hline \multicolumn{7}{|l|}{ Moraceae Gaudich. } \\
\hline Artocarpus chama Buch.-Ham. & Chapalish & Tree, $1 ; \mathrm{pl}$ & Rs, Wl & Ko, Ss & Fr, $\mathrm{T}$ & MAR 2920 \\
\hline A. heterophyllus Lam. & Kanthal & Tree, m; pl & Hs, Wl & All upazilas & Fr, $\mathrm{T}$ & MAR 2921 \\
\hline A. lakoocha Roxb. & Dewa & Tree, m; pl & $\mathrm{Wl}$ & $\mathrm{De}, \mathrm{Ka}, \mathrm{Ko}, \mathrm{Ss}$ & $\mathrm{Fr}, \mathrm{T}$ & MAR 2922 \\
\hline Ficus benghalensis L. & Bot & Tree, $1 ; \mathrm{w}$ & $\mathrm{Fl}, \mathrm{Rs}, \mathrm{Wl}$ & All upazilas ${ }^{+}$ & $\mathrm{O}, \mathrm{Fw}$ & MAR 2923 \\
\hline F. elastica Roxb. ex Hornem. & Rubber bot & Tree, $\mathrm{m} ; \mathrm{pl}$ & $\mathrm{Fl}, \mathrm{Rs}$ & $\mathrm{Ka}, \mathrm{Sh}^{+}, \mathrm{Ss}$ & $\mathrm{O}$ & MAR 2924 \\
\hline F. heterophylla L. f. & Bhuidumur & Shrub; w & $\mathrm{Sj}, \mathrm{Wl}$ & $\mathrm{De}, \mathrm{Ka}, \mathrm{Ko}$ & M & MAR 2925 \\
\hline F. hispida L. f. & Kakdumur & Tree, s; w & $\mathrm{Sj}, \mathrm{Wl}$ & All upazilas & $\mathrm{M}, \mathrm{Vg}$ & MAR 2926 \\
\hline F. racemosa $\mathrm{L}$. & Jagdumur & Tree, 1 ; w & $\mathrm{Sj}, \mathrm{Fl}$ & $\mathrm{De}, \mathrm{Ka}, \mathrm{Ss}$ & M & MAR 2927 \\
\hline F. religiosa $\mathrm{L}$. & Ashwath & Tree, $1 ; \mathrm{w}$ & $\mathrm{Wl}, \mathrm{Sj}$ & $\mathrm{De}, \mathrm{As}, \mathrm{Ss}$ & $\mathrm{M}, \mathrm{O}$ & MAR 2928 \\
\hline F. rumphii Blume & Khiri bot & Tree, $1 ; \mathrm{w}$ & $\mathrm{Fl}, \mathrm{Rs}, \mathrm{Wl}$ & $\mathrm{Ko}, \mathrm{Sh}^{+}, \mathrm{Ss}, \mathrm{Ta}$ & $\mathrm{M}, \mathrm{O}$ & MAR 2929 \\
\hline Morus alba $\mathrm{L}$. & Shada tut & Tree, s; pl & Ml, Rs & As, Ko, Ss & $\mathrm{Fr}, \mathrm{Fw}$ & MAR 2930 \\
\hline Streblus asper Lour. & Sheora & Tree, 1 ; w & $\mathrm{Sj}, \mathrm{Wl}$ & All upazilas ${ }^{+}$ & Fw, M & MAR 2931 \\
\hline \multicolumn{7}{|l|}{ Urticaceae Juss. } \\
\hline Laportea interrupta (L.) Chew & Chotrapatta & Herb, cl; w & $\mathrm{Sj} \mathrm{Wl}$ & $\mathrm{De}, \mathrm{Ka}, \mathrm{Ss}$ & M & KMNI 002 \\
\hline Pilea microphylla (L.) Liebm. & Latamaricha & Herb, pr; w & Obw & All upazilas ${ }^{+}$ & M & KMNI 003 \\
\hline $\begin{array}{l}\text { Pouzolzia zeylanica (L.) Benn. } \\
\text { Casuarinaceae R. Br. }\end{array}$ & Kullaruki & Herb, er; w & $\mathrm{Fl}, \mathrm{Gl}, \mathrm{Rs}$ & All upazilas ${ }^{+}$ & M & KMNI 004 \\
\hline $\begin{array}{l}\text { Casuarina equisetifolia L. } \\
\text { Nyctaginaceae Juss. }\end{array}$ & Jhaw & Tree, $1 ; \mathrm{pl}$ & Ml, Rs & Ko, Ss & $\mathrm{O}$ & GMH 5220 \\
\hline Boerhavia diffusa $\mathrm{L}$. & Punarnava & Herb, pr; w & $\mathrm{Fl}, \mathrm{Rs}$ & De, Ko, Ss & M & GMH 5217 \\
\hline Bougainvillea spectabilis Willd. & Baganbilash & Shrub, li; pl & $\mathrm{Hs}$ & All upazilas ${ }^{+}$ & $\mathrm{O}$ & GMH 5223 \\
\hline $\begin{array}{l}\text { Mirabilis jalapa } \mathrm{L} \text {. } \\
\text { Aizoaceae Martinov }\end{array}$ & Sandhyamoni & Herb, er; pl & $\mathrm{Fl}, \mathrm{Hs}$ & As, De, Ko, Ss & $\mathrm{M}, \mathrm{O}$ & GMH 5224 \\
\hline $\begin{array}{l}\text { Trianthema portulacastrum L. } \\
\text { Cactaceae Juss. }\end{array}$ & Swetpunarnova & Herb, pr; w & Af, Fl, Rs & As, Ko, Ta & $\mathrm{M}, \mathrm{Gm}$ & MAR 2932 \\
\hline Opuntia ficus-indica (L.) Mill. & Fhonimonosha & Shrub; w & Ml, Rs & As, De, Sh & $\mathrm{He}, \mathrm{M}$ & KMNI 015 \\
\hline O. stricta (Haw.) Haw. & Nagphana & Shrub; w & Ml, Rs & $\mathrm{As}, \mathrm{Sh}, \mathrm{Ka}$ & $\mathrm{He}, \mathrm{M}$ & KMNI 016 \\
\hline \multicolumn{7}{|l|}{ Amaranthaceae Juss. } \\
\hline Achyranthes aspera $\mathrm{L}$. & Apang & Herb, er; w & $\mathrm{Fl}, \mathrm{Rs}, \mathrm{Wl}$ & All upazilas & M & GMH 5230 \\
\hline Aerva lanata (L.) Juss. & Chaya & Herb, pr; w & $\mathrm{Fl}$, Rs & Ss, Ta & $\mathrm{M}, \mathrm{Vg}$ & GMH 5225 \\
\hline $\begin{array}{l}\text { Alternanthera bettzickiana (Regel) } \\
\text { G. Nicholson }\end{array}$ & Calico gach & Herb, pr; w & $\mathrm{Fl}, \mathrm{Rs}$ & Ss & $\mathrm{M}, \mathrm{Vg}$ & MAR 2933 \\
\hline A. paronychioides A. St.-Hil. & Jhuli khata & Herb, pr; w & Af, Fl, Rs & As, Sh, Ta & $\mathrm{M}, \mathrm{Vg}$ & GMH 5366 \\
\hline A. philoxeroides (Mart.) Griseb. & Henchi & Herb, fr; w & Af, Wtl & All upazilas ${ }^{+}$ & $\mathrm{Gm}, \mathrm{Vg}$ & GMH 5226 \\
\hline A. sessilis (L.) R. Br. ex DC. & Malancha & Herb, pr; w & Af, Fl, Rs & All upazilas ${ }^{+}$ & $\mathrm{M}, \mathrm{Vg}$ & GMH 5227 \\
\hline Amaranthus blitum $\mathrm{L}$. & Goburanotey & Herb, er; w & $\mathrm{Fl}, \mathrm{Rs}$ & As, De, Ss, Ta & $\mathrm{M}, \mathrm{Vg}$ & MAR 2935 \\
\hline A. spinosus $\mathrm{L}$. & Kantanotey & Herb, er; w & Af, Fl, Rs & All upazilas & $\mathrm{M}, \mathrm{Vg}$ & MAR 2936 \\
\hline A. tricolor $\mathrm{L}$. & Lalshak & Herb, er; cv & Af/Apl & All upazilas ${ }^{+}$ & $\mathrm{Vg}$ & MAR 2937 \\
\hline A. viridis $\mathrm{L}$. & Notey shak & Herb, er; w & Af, Fl, Rs & All upazilas & $\mathrm{M}, \mathrm{Vg}$ & GMH 5367 \\
\hline Celosia argentea $\mathrm{L}$. & Morog phul & Herb, er; pl & Hs, Rs & $\mathrm{De}, \mathrm{Ss}, \mathrm{Ta}$ & $\mathrm{M}, \mathrm{O}$ & GMH 5229 \\
\hline Chenopodium album $\mathrm{L}$. & Botua shak & Herb, er; w & Af, Fl, Rs & As, Ko, Ss, Ta & $\mathrm{M}, \mathrm{Vg}$ & GMH 5228 \\
\hline Cyathula prostrata (L.) Blume & Shyontula & Herb, pr; w & $\mathrm{Fl}, \mathrm{Rs}$ & As, De, Ka, Ss & M & GMH 5231 \\
\hline Gomphrena celosioides Mart. & Bottam phul & Herb, pr; w & $\mathrm{Gl}, \mathrm{Fl}, \mathrm{Rs}$ & Ss, Ta & M & GMH 5235 \\
\hline \multicolumn{7}{|l|}{ Portulacaceae Juss. } \\
\hline Portulaca oleracea $\mathrm{L}$. & Boronunia & Herb, pr; w & Af, Fl, Rs & $\mathrm{Ko}, \mathrm{Sh}^{+}, \mathrm{Ss}$ & $\mathrm{M}, \mathrm{Vg}$ & KMNI 005 \\
\hline P. grandiflora Hook. & Time phul & Herb, pr; pl & Hs, Rs & $\mathrm{De}, \mathrm{Ka}, \mathrm{Ss}$ & $\mathrm{O}$ & KMNI 006 \\
\hline
\end{tabular}


Table 1 Contd.

\begin{tabular}{|c|c|c|c|c|c|c|}
\hline Scientific name & Bangla name & Habit & Habitat & Distribution & Use & RSE \\
\hline \multicolumn{7}{|l|}{ Basellaceae Raf. } \\
\hline Basella alba $\mathrm{L}$. & Pui shak & Herb, cr; cv & Af/Apl, Hs & All upazilas ${ }^{+}$ & $\mathrm{Vg}$ & GMH 5240 \\
\hline \multicolumn{7}{|l|}{ Molluginaceae Bartl. } \\
\hline Glinus lotoides L. & Alu ghash & Herb, pr; w & Af, Fl & $\mathrm{Ka}, \mathrm{Ko}, \mathrm{Ss}$ & M & GMH 5232 \\
\hline G. oppositifolius (L.) Aug. DC. & Gima shak & Herb, pr; w & Af, Fl & Ko, Ss & $\mathrm{M}, \mathrm{Vg}$ & GMH 5236 \\
\hline $\begin{array}{l}\text { Trigastrotheca pentaphylla (L.) } \\
\text { Thulin }\end{array}$ & Khetpapra & Herb, pr; w & Af, $\mathrm{Fl}$ & Ko, Ss & $\mathrm{M}$ & GMH 5239 \\
\hline \multicolumn{7}{|l|}{ Polygonaceae Juss. } \\
\hline Persicaria barbata (L.) H. Hara & Biskatali & Herb, er; w & $\mathrm{Fl}, \mathrm{Wtl}$ & $\mathrm{De}, \mathrm{Ko}, \mathrm{Sh}, \mathrm{Ss}$ & M & MAR 2938 \\
\hline P. glabra (Willd.) M. Gómez & Lal kukri & Herb, er; w & Af, $\mathrm{Fl}$ & $\mathrm{Ka}, \mathrm{Sh}^{+}, \mathrm{Ta}$ & M & MAR 2939 \\
\hline P. hydropiper (L.) Delarbre & Panibiskatali & Herb, er; w & Af, Fl, Wtl & As, Ko, Ta & M & MAR 2940 \\
\hline P. orientalis (L.) Spach & Bara panimarich & Herb, er; w & $\mathrm{Fl}, \mathrm{Wtl}$ & As, Ss, Ta & M & MAR 2941 \\
\hline Polygonum effusum Meisn. & Rani phul & Herb, er; w & Wtl & Ko, Ss, Ta & M & MAR 2942 \\
\hline Rumex dentatus $\mathrm{L}$. & Bon-palang & Herb, er; w & Af, Fl, Rs & Ko, Ss, Sh & M & MAR 2943 \\
\hline R. maritimus $\mathrm{L}$. & Dati-palang & Herb, er; w & Af, Fl, Rs & $\mathrm{De}, \mathrm{Sh}, \mathrm{Ta}$ & M & MAR 2944 \\
\hline \multicolumn{7}{|l|}{ Plumbaginaceae Juss. } \\
\hline $\begin{array}{l}\text { *Aegialitis rotundifolia Roxb. } \\
\text { Dilleniaceae Salisb. }\end{array}$ & Nunia & Shrub; w & Fm, Wl & $\mathrm{Sh}^{+}$ & Fw, M & GMH 5233 \\
\hline \multicolumn{7}{|l|}{ Clusiaceae Lindl. } \\
\hline *Calophyllum inophyllum $\mathrm{L}$. & Punnul & Tree, m; w & Fm, Rs & $\mathrm{Sh}^{+}$ & $\mathrm{M}, \mathrm{Oy}$ & MSR 3320 \\
\hline $\begin{array}{l}\text { Mesua ferrea } \mathrm{L} \text {. } \\
\text { Elaeocarpaceae Juss. }\end{array}$ & $\mathrm{Nag}$ & Tree & Rs & De, 1 & M, O & KMNI 008 \\
\hline $\begin{array}{l}\text { Elaeocarpus floribundus Blume } \\
\text { Sterculiaceae Vent. }\end{array}$ & Jalpai & Tree, $\mathrm{m} ; \mathrm{pl}$ & Hs & $\mathrm{De}, \mathrm{Ka}, \mathrm{Ko}, \mathrm{Ss}$ & $\mathrm{Fr}, \mathrm{Oy}$ & MAR 3010 \\
\hline Abroma augusta (L.) L. f. & Ulatkambal & Shrub; w & $\mathrm{Hs}, \mathrm{Sj}$ & As, Sh, Ss & $\mathrm{Fb}, \mathrm{M}$ & $S A K 2112$ \\
\hline Melochia corchorifolia $\mathrm{L}$. & Tiki-okra & Shrub; w & Wl & Ss & M & $S A K 2113$ \\
\hline Sterculia foetida $\mathrm{L}$. & Baksho badam & Tree, $1 ; \mathrm{pl}$ & Hs, Rs & $\mathrm{Ka}, \mathrm{Sh}^{+}$ & Fr, M & MSR 3319 \\
\hline \multicolumn{7}{|l|}{ Bombacaceae Kunth. } \\
\hline $\begin{array}{l}\text { Bombax ceiba } \mathrm{L} \text {. } \\
\text { Malvaceae Juss. }\end{array}$ & Shimul & Tree, l; w & Rs, Wl & All upazilas & $\mathrm{Fb}, \mathrm{M}$ & MAR 2949 \\
\hline $\begin{array}{l}\text { Abelmoschus esculentus (L.) } \\
\text { Moench }\end{array}$ & Dherosh & Herb, er; cv & Af & Ko, Sh, Ta & $\mathrm{Vg}$ & GMH 5237 \\
\hline Abutilon indicum (L.) Sweet & Petari & Shrub; w & $\mathrm{Fl}, \mathrm{Rs}, \mathrm{Sj}$ & All upazilas & $\mathrm{Fb}, \mathrm{M}$ & GMH 5234 \\
\hline *Brownlowia tersa (L.) Kosterm. & Lata sundri & Shrub; w & $\mathrm{Rb}, \mathrm{Wl}$ & $\mathrm{Sh}^{+}$ & Fw, M & GMH 5241 \\
\hline Ceiba pentandra (L.) Gaertn. & Shada shimul & Tree, $\mathrm{m} ; \mathrm{pl}$ & Hs, Rs & As, $\mathrm{Sh}$ & $\mathrm{Fb}, \mathrm{T}$ & GMH 5249 \\
\hline Corchorus aestuans $\mathrm{L}$. & Janglipat & Shrub; w & $\mathrm{Fl}, \mathrm{Rs}, \mathrm{Sj}$ & $\mathrm{Ko}, \mathrm{Ss}, \mathrm{Sh}^{+}$ & $\mathrm{Fb}, \mathrm{M}$ & GMH 5245 \\
\hline Gossypium arboreum $\mathrm{L}$. & Karpash & Shrub; cv & Af & $\mathrm{De}, \mathrm{Ko}, \mathrm{Sh}$ & $\mathrm{Fb}, \mathrm{Oy}$ & GMH 5242 \\
\hline Grewia asiatica $\mathrm{L}$. & Pholsa & Tree, s; pl & $\mathrm{Hs}, \mathrm{Wl}$ & $\mathrm{Ka}$ & Fr, M & MAR 2945 \\
\hline G. glandulosa Vahl & Pathaka & Tree, s; w & $\mathrm{Sj}$ & $\mathrm{Ka}$ & M & MAR 2946 \\
\hline *Heritiera fomes Buch.-Ham. & Sundri & Tree, $1 ; \mathrm{w}$ & Wl & $\mathrm{Sh}^{+}$ & $\mathrm{T}$ & GMH 5250 \\
\hline Hibiscus rosa-sinensis $\mathrm{L}$. & Joba & Shrub; pl & Hs, Rs & All upazilas & $\mathrm{O}$ & GMH 5246 \\
\hline H. sabdariffa $\mathrm{L}$. & Chukar & Shrub; pl & Hs, Ml & Ko, Ss, Sh, Ta & M & GMH 5247 \\
\hline H. schizopetalus (Dyer) Hook. f. & Jhumko jaba & Shrub; pl & Hs, Rs & Ss & $\mathrm{O}$ & GMH 5243 \\
\hline *H. tiliaceus $\mathrm{L}$. & Bhola & Shrub, sc; w & $\mathrm{Rb}, \mathrm{Wl}$ & $\mathrm{Sh}^{+}$ & $\mathrm{Fb}, \mathrm{Fw}$ & GMH 5244 \\
\hline H. vitifolius $\mathrm{L}$. & Bonkarpas & Shrub; w & $\mathrm{Sj}$ & Ss & $\mathrm{M}, \mathrm{O}$ & GMH 5251 \\
\hline Malvaviscus arboreus Cav. & Morich joba & Shrub; pl & Hs, Rs & $\mathrm{De}, \mathrm{Ka}, \mathrm{Ss}$ & $\mathrm{O}$ & GMH 5255 \\
\hline Microcos tomentosa $\mathrm{Sm}$. & Asar & Shrub; w & $\mathrm{Sj}, \mathrm{Wl}$ & Ka, Ko & Fw, M & MAR 2947 \\
\hline
\end{tabular}


Table 1 Contd.

\begin{tabular}{|c|c|c|c|c|c|c|}
\hline Scientific name & Bangla name & Habit & Habitat & Distribution & Use & RSE \\
\hline Pentapetes phoenicea $\mathrm{L}$. & Bandhuli phul & Shrub; pl & Hs & Ko, Sh, Ta & $\mathrm{O}$ & GMH 5466 \\
\hline Sida acuta Burm. f. & Kureta & Herb, er; w & $\mathrm{Fl}, \mathrm{Sj}, \mathrm{Rs}$ & All upazilas ${ }^{+}$ & M & GMH 5260 \\
\hline S. cordata (Burm. f.) Bross. Waalk. & Pitberela & Herb, er; w & $\mathrm{Fl}, \mathrm{Sj}, \mathrm{Rs}$ & All upazilas ${ }^{+}$ & M & GMH 5256 \\
\hline S. cordifolia $\mathrm{L}$ & & Herb, er; w & $\mathrm{Fl}, \mathrm{Rs}$ & $\mathrm{Ka}, \mathrm{Sh}, \mathrm{Ss}$ & M & GMH 5252 \\
\hline S. rhombifolia $\mathrm{L}$. & Lal-berela & Herb, er; w & $\mathrm{Fl}, \mathrm{Rs}$ & As, De, Sh, Ta & $\mathrm{Fb}, \mathrm{M}$ & GMH 5259 \\
\hline $\begin{array}{l}\text { *Thespesia populnea }(\text { L.) Sol. ex } \\
\text { Corrêa }\end{array}$ & Porosh pipul & Tree, s; w & $\mathrm{Fm}, \mathrm{Rb}$ & $\mathrm{Sh}^{+}$ & $\mathrm{Fb}, \mathrm{M}$ & MSR 3308 \\
\hline Triumfetta rhomboidea Jacq. & Bon okra & Shrub; w & $\mathrm{Fl}, \mathrm{Rs}, \mathrm{Sj}$ & As, Ko, Ss, Ta & $\mathrm{Fb}, \mathrm{M}$ & MAR 2948 \\
\hline Urena lobata $\mathrm{L}$. & Banokra & Shrub; w & $\mathrm{Fl}, \mathrm{Sj}$, Rs & All upazilas & $\mathrm{Fb}, \mathrm{M}$ & GMH 5257 \\
\hline $\begin{array}{l}\text { Wissadula periplocifolia (L.) } \\
\text { Thwaites }\end{array}$ & Shada nagmoni & Shrub; w & $\mathrm{Rs}, \mathrm{Sj}$ & $\mathrm{De}, \mathrm{Ka}, \mathrm{Ss}$ & $\mathrm{Fb}, \mathrm{M}$ & GMH 5261 \\
\hline \multicolumn{7}{|l|}{ Lecythidaceae A. Rich. } \\
\hline $\begin{array}{l}\text { Barringtonia acutangula (L.) } \\
\text { Gaertn. }\end{array}$ & Hijal & Tree, m; w & $\mathrm{Ml}, \mathrm{Sj}, \mathrm{Wtl}$ & De & $\mathrm{M}, \mathrm{O}$ & GMH 5254 \\
\hline \multicolumn{7}{|l|}{ Tamaricaceae Link } \\
\hline $\begin{array}{l}\text { *Tamarix indica Willd. } \\
\text { Passifloraceae Juss. ex Roussel }\end{array}$ & Nona jhaw & Tree, s; w & Fm, Wl & $\mathrm{Sh}^{+}$ & Fw, M & GMH 5262 \\
\hline Passiflora edulis Sims & Passion phal & Herb, cl; pl & Hs & Ss & $\mathrm{Fr}$ & $S A K 2114$ \\
\hline P. foetida $\mathrm{L}$. & Jhumka lata & Herb, cl; w & $\mathrm{Sj}$ & Ka, Ko, Ta & Fr, M & SAK 2115 \\
\hline P. suberosa $\mathrm{L}$. & Mela jhumka & Herb, cl; w & Rs & De, Ta & M & SAK 2116 \\
\hline Turnera ulmifolia $\mathrm{L}$. & Bashanti & Herb, er; w & Rs & Sh & M & $S A K 2117$ \\
\hline \multicolumn{7}{|l|}{ Caricaceae Dumort. } \\
\hline Carica papaya $\mathrm{L}$. & Papya & Tree, $\mathrm{m} ; \mathrm{pl}$ & Af, Hs, Rs & All upazilas ${ }^{+}$ & Fr, $\mathrm{Vg}$ & GMH 5263 \\
\hline \multicolumn{7}{|l|}{ Cucurbitaceae Juss. } \\
\hline Benincasa hispida (Thunb.) Cogn. & Chalkumra & Herb, cl; cv & Af, Hs & Ko, Ss & $\mathrm{Vg}$ & MAR 2952 \\
\hline $\begin{array}{l}\text { Citrullus lanatus (Thunb.) Matsum. } \\
\& \text { Nakai }\end{array}$ & Tormuj & Herb, cl; cv & Af & De, Ka, Ko, Sh & $\mathrm{Fr}$ & MAR 2953 \\
\hline Coccinia grandis (L.) Voigt & Telakucha & Herb, cl; w & $\mathrm{Sj}, \mathrm{Wl}$ & All upazilas & $\mathrm{M}, \mathrm{Vg}$ & MAR 2954 \\
\hline Cucumis melo $\mathrm{L}$. & Bangi & Herb, cl; cv & Af & Ka & Fr, $\mathrm{Vg}$ & MAR 2955 \\
\hline C. sativus $\mathrm{L}$. & Khira, Shosha & Herb, cl; cv & Af, Hs & $\mathrm{De}, \mathrm{Ka}, \mathrm{Ko}$ & Fr, $\mathrm{Vg}$ & MAR 2956 \\
\hline Cucurbita maxima Duchesne & Misti kumra & Herb, cl; cv & Af, Hs & All upazilas ${ }^{+}$ & $\mathrm{M}, \mathrm{Vg}$ & MAR 2957 \\
\hline $\begin{array}{l}\text { Gymnopetalum chinense (Lour.) } \\
\text { Merr. }\end{array}$ & Banpatol & Herb, cl; w & $\mathrm{Sj}$ & As, De, Ss, Ta & M & MAR 2958 \\
\hline $\begin{array}{l}\text { Lagenaria siceraria (Molina) } \\
\text { Standl. }\end{array}$ & Lao & Herb, cl; cv & Af, Hs & All upazilas ${ }^{+}$ & $\mathrm{M}, \mathrm{Vg}$ & MAR 2959 \\
\hline Luffa acutangula (L.) Roxb. & Jhinga & Herb, cl; cv & Af, Hs & All upazilas & $\mathrm{M}, \mathrm{Vg}$ & MAR 2960 \\
\hline L. cylindrica (L.) M. Roem. & Dhundal & Herb, cl; cv & Af, Hs & All upazilas & $\mathrm{M}, \mathrm{Vg}$ & MAR 2961 \\
\hline Momordica dioica Roxb. ex Willd. & Kakroll & Herb, cl; cv & Af, Hs & All upazilas & $\mathrm{M}, \mathrm{Vg}$ & MAR 2962 \\
\hline M. charantia $\mathrm{L}$. & Korolla & Herb, cl; cv & Af, Hs & Ka, Ko & $\mathrm{M}, \mathrm{Vg}$ & MAR 2963 \\
\hline $\begin{array}{l}\text { Mukia maderaspatana (L.) M. } \\
\text { Roem. }\end{array}$ & Agmukhi & Herb, cl; w & Af, $\mathrm{Fl}, \mathrm{Sj}$ & As, De, Ss, Ta & M & MAR 2964 \\
\hline $\begin{array}{l}\text { Solena amplexicaulis (Lam.) } \\
\text { Gandhi }\end{array}$ & Rakhal sosha & Herb, cl; w & $\mathrm{Sj}$ & $\mathrm{De}, \mathrm{Ka}$ & M & MAR 2965 \\
\hline Trichosanthes dioica Roxb. & Potol & Herb, cl; cv & Af, Hs & De, Ko, Ta & $\mathrm{M}, \mathrm{Vg}$ & MAR 2966 \\
\hline T. cucumerina $\mathrm{L}$. & Chichinga & Herb, cl; cv & Af, Hs & $\mathrm{De}, \mathrm{Ka}, \mathrm{Ko}, \mathrm{Sh}$ & $\mathrm{M}, \mathrm{Vg}$ & MAR 2967 \\
\hline T. tricuspidata Lour. & Makal & Herb, cl; w & $\mathrm{Fm}, \mathrm{Sj}$ & $\mathrm{De}, \mathrm{Ka}$ & M & MAR 2968 \\
\hline \multicolumn{7}{|l|}{ Salicaceae Mirb. } \\
\hline Casearia tomentosa Roxb. & Bhari, Chilla & Tree, s; w & Fm, Wl & $\mathrm{De}$ & $\mathrm{Fp}, \mathrm{M}$ & MAR 2950 \\
\hline Flacourtia indica (Burm. f.) Merr. & Bauchi & Shrub; w & $\mathrm{Sj}, \mathrm{Wl}$ & $\mathrm{De}, \mathrm{Ko}, \mathrm{Ss}$ & Fr, M & MAR 2951 \\
\hline
\end{tabular}


Table 1 contd.

\begin{tabular}{|c|c|c|c|c|c|c|}
\hline Scientific name & Bangla name & Habit & Habitat & Distribution & Use & RSE \\
\hline \multicolumn{7}{|l|}{ Capparaceae Juss. } \\
\hline Capparis zeylanica $\mathrm{L}$. & Katai & Shrub, sc; w & $\mathrm{Sj}$ & $\mathrm{De}, \mathrm{Ka}, \mathrm{Ko}, \mathrm{Ss}$ & M & GMH 5264 \\
\hline \multicolumn{7}{|l|}{ Cleomaceae Bercht. \& J. Pres1 } \\
\hline Cleome rutidosperma DC. & Nil hurhurey & Herb, er; w & Af, Fl, Rs & All upazilas & M & GMH 5268 \\
\hline C. viscosa $\mathrm{L}$. & Halud hurhurey & Herb, er; w & Af, Fl, Rs & All upazilas & $\mathrm{M}, \mathrm{Vg}$ & GMH 5271 \\
\hline \multicolumn{7}{|l|}{ Brassicaceae Burnett } \\
\hline Brassica napus $\mathrm{L}$. & Sarisha & Herb, er; cv & Af, $\mathrm{Hs}$ & De, Ko, Ta & Lf, Oy & MAR 2969 \\
\hline B. oleracea var. botrytis $\mathrm{L}$. & Fulkopie & Herb, er; cv & Af & Ko & $\mathrm{Vg}$ & MAR 2970 \\
\hline B. oleracea var. capitata $\mathrm{L}$. & Badhakopie & Herb, er; cv & Af & Ko & Lf, $\mathrm{Vg}$ & MAR 2971 \\
\hline Cardamine flexuosa With. & Bansarisha & Herb, er; w & Af, Fl & Ss, Ta & M & MAR 2972 \\
\hline Daucus carota $\mathrm{L}$. & Gajor & Herb, er; cv & Af & $\mathrm{De}, \mathrm{Ka}, \mathrm{Ko}$ & $\mathrm{Vg}$ & MAR 2973 \\
\hline $\begin{array}{l}\text { Raphanus raphanistrum subsp. } \\
\text { sativus (L.) Domin }\end{array}$ & Mula & Herb, er; cv & Af & Ka, Ko Ss, Ta & $\mathrm{Vg}$ & MAR 2974 \\
\hline Rorippa indica (L.) Hiern & Bansarisha & Herb, er; w & $\mathrm{Fl}, \mathrm{Ml}$ & $\mathrm{Ka}, \mathrm{Sh}, \mathrm{Ss}$ & $\mathrm{M}, \mathrm{Vg}$ & MAR 2975 \\
\hline \multicolumn{7}{|l|}{ Moringaceae Martinov } \\
\hline Moringa oleifera Lamk. & Shajna & Tree, $\mathrm{m} ; \mathrm{pl}$ & Hs, Ml, Rs & All upazilas & $\mathrm{M}, \mathrm{Vg}$ & GMH 5265 \\
\hline \multicolumn{7}{|l|}{ Sapotaceae Juss. } \\
\hline $\begin{array}{l}\text { Madhuca longifolia (J. König ex } \\
\text { L.) J.F. Macbr. }\end{array}$ & Mohua & Tree, m; w & Rs, Wl & $\mathrm{Ka}, \mathrm{Sh}^{+}, \mathrm{Ss}$ & $\mathrm{M}, \mathrm{Oy}$ & SAK 2118 \\
\hline Manilkara zapota (L.) P. Royen & Sopheda & Tree, $\mathrm{m} ; \mathrm{pl}$ & Hs & All upazilas ${ }^{+}$ & Fr, M & SAK 2119 \\
\hline Mimusops elengi $\mathrm{L}$. & Bokul & Tree, $\mathrm{m} ; \mathrm{pl}$ & Rs & $\mathrm{De}, \mathrm{Ka}, \mathrm{Ss}$ & $\mathrm{M}, \mathrm{O}$ & SAK 2120 \\
\hline \multicolumn{7}{|l|}{ Ebenaceae Gürke } \\
\hline Diospyros discolor Willd. & Bilati gab & Tree, $\mathrm{m} ; \mathrm{pl}$ & Hs, Rs & $\mathrm{As}, \mathrm{De}, \mathrm{Sh}^{+}, \mathrm{Ss}$ & Fr, M & SAK 2121 \\
\hline D. malabarica (Desr.) Kostel. & Deshi gab & Tree, $\mathrm{m} ; \mathrm{w}$ & Wl & $\mathrm{Ka}$ & Fr, M & SAK 2122 \\
\hline \multicolumn{7}{|l|}{ Myrsinaceae R. Br. } \\
\hline Ardisia solanacea (Poir.) Roxb. & Banjam & Shrub; w & $\mathrm{Wl}$ & Ka, Ko & $\mathrm{M}, \mathrm{O}$ & GMH 5267 \\
\hline \multicolumn{7}{|l|}{ Primulaceae Batsch } \\
\hline $\begin{array}{l}\text { *Aegiceras corniculatum }(\mathrm{L} .) \\
\text { Blanco }\end{array}$ & Kholshi & Shrub; w & Wl & $\mathrm{Sh}^{+}$ & $\mathrm{Hp}, \mathrm{Fw}$ & GMH 5266 \\
\hline \multicolumn{7}{|l|}{ Crassulaceae J. St.-Hil. } \\
\hline Kalachoe pinnata (Lam.) Pers. & Patharkuchi & Herb, er; pl & Hs & $\mathrm{De}, \mathrm{Ko}, \mathrm{Sh}, \mathrm{Ss}$ & $\mathrm{M}, \mathrm{O}$ & GMH 5270 \\
\hline \multicolumn{7}{|l|}{ Rosaceae Juss. } \\
\hline Rosa centifolia $\mathrm{L}$. & Golap & Shrub; pl & Hs & Ss & $\mathrm{M}, \mathrm{O}$ & GMH 5420 \\
\hline R. chinensis Jacq. & Jangli golap & Shrub; pl & Hs, Ml & Ss & $\mathrm{He}, \mathrm{M}$ & KMNI 038 \\
\hline \multicolumn{7}{|l|}{ Mimosaceae R. Br. } \\
\hline Acacia auriculiformis Benth. & Akashmoni & Tree, $1 ; \mathrm{pl}$ & $\mathrm{Fl}, \mathrm{Rs}, \mathrm{Wl}$ & All upazilas ${ }^{+}$ & $\mathrm{T}$ & MAR 2976 \\
\hline A. mangium Willd. & Mangium & Tree, $1 ; \mathrm{pl}$ & Rs, Wl & $\mathrm{Ka}, \mathrm{Ss}$ & $\mathrm{T}$ & MAR 2977 \\
\hline A. nilotica (L.) Delile & Babla & Tree, $\mathrm{m} ; \mathrm{w}$ & $\mathrm{Fl}, \mathrm{Rs}$ & All upazilas ${ }^{+}$ & $\mathrm{Gu}, \mathrm{M}$ & MAR 2978 \\
\hline Albizia procera (Roxb.) Benth. & Sil koroi & Tree, $1 ; \mathrm{w}$ & Rs, Wl & As, De, Ka, Ta & $\mathrm{T}$ & MAR 2979 \\
\hline $\begin{array}{l}\text { A. richardiana (Voigt) King \& } \\
\text { Prain }\end{array}$ & Raj siris & Tree, $1 ; \mathrm{pl}$ & Rs & $\mathrm{As}, \mathrm{Sh}^{+}, \mathrm{Ta}$ & $\mathrm{T}$ & MAR 2980 \\
\hline A. saman (Jacq.) Merr. & Shirish & Tree, $1 ; \mathrm{pl}$ & Ml, Rs, Wl & All upazilas ${ }^{+}$ & $\mathrm{T}$ & MAR 2981 \\
\hline *Cynometra ramiflora $\mathrm{L}$. & Shigra & Tree, s; w & Fm, Wl & $\mathrm{Sh}^{+}$ & Fw, M & GMH 5268 \\
\hline Entada rheedii Spreng. & Gila lata & Shrub, li; w & Fm, Wl & $\mathrm{Ka}$ & $\mathrm{M}, \mathrm{Wp}$ & MAR 2982 \\
\hline $\begin{array}{l}\text { Leucaena leucocephala (Lam.) de } \\
\text { Wit }\end{array}$ & Ipil-ipil & Tree, $1 ; \mathrm{w}$ & $\mathrm{Fl}, \mathrm{Rs}, \mathrm{Wl}$ & All upazilas & $\mathrm{T}$ & KMNI 009 \\
\hline Mimosa pudica $\mathrm{L}$. & Lajjaboti & Herb, pr; w & $\mathrm{Gl}, \mathrm{Fl}, \mathrm{Rs}$ & All upazilas ${ }^{+}$ & M & KMNI 010 \\
\hline Pithecellobium dulce (Roxb.) Benth. & Khoia babla & Tree, $\mathrm{m} ; \mathrm{w}$ & Hs, Rs & $\mathrm{As}, \mathrm{Sh}^{+}, \mathrm{Ss}$ & Fr, M & GMH 5272 \\
\hline Prosopis juliflora (Sw.) DC. & Bilati babla & Tree, $\mathrm{m} ; \mathrm{w}$ & Rs & $\mathrm{Sh}$ & Fw, M & GMH 5273 \\
\hline
\end{tabular}


Table 1 contd.

\begin{tabular}{|c|c|c|c|c|c|c|}
\hline Scientific name & Bangla name & Habit & Habitat & Distribution & Use & RSE \\
\hline \multicolumn{7}{|l|}{ Caesalpiniaceae R. Br. } \\
\hline Caesalpinia bonduc (L.) Roxb. & Nata kanta & Shrub, sc; w & $\mathrm{Sj}, \mathrm{Fm}$ & Ko, Ta & M, Oy & SAK 2123 \\
\hline Cassia fistula $\mathrm{L}$. & Badarlathi & Tree, $\mathrm{m} ; \mathrm{w}$ & Ml, Rs & As, De, Ka, Ss & $\mathrm{M}, \mathrm{O}$ & SAK 2124 \\
\hline $\begin{array}{l}\text { C. javanica subsp. nodosa (Roxb.) } \\
\text { K. Larsen \& S.S. Larsen }\end{array}$ & Burmese shonalu & Tree, $\mathrm{m} ; \mathrm{pl}$ & Ml, Rs & Ss & $\mathrm{O}$ & $S A K 2125$ \\
\hline Delonix regia (Hook.) Raf. & Krishnachura & Tree, $1 ; \mathrm{pl}$ & Rs & All upazilas & $\mathrm{M}, \mathrm{O}$ & SAK 2126 \\
\hline Parkinsonia aculeata $\mathrm{L}$. & Bilati babla & Tree, s; pl & Rs & As, $\mathrm{Sh}$ & Fw, M & SAK 2127 \\
\hline $\begin{array}{l}\text { Peltophorum pterocarpum (DC.) } \\
\text { K. Heyne }\end{array}$ & Radha chura & Tree, $1 ; \mathrm{pl}$ & Rs & Ss, Ta & $\mathrm{M}, \mathrm{O}$ & SAK 2128 \\
\hline Saraca asoca (Roxb.) Willd. & Ashok & Tree, $\mathrm{m} ; \mathrm{pl}$ & Rs, Wl & Ss & $\mathrm{M}, \mathrm{O}$ & SAK 2129 \\
\hline Senna alata (L.) Roxb. & Dadmardan & Shrub; w & $\mathrm{Fl}, \mathrm{Hs}, \mathrm{Rs}$ & As, Ss, Sh & M & $S A K 2130$ \\
\hline S. occidentalis (L.) Link & Barakalkesunda & Shrub; w & $\mathrm{Fl}$, Rs & As, Ko, Sh, Ta & M & SAK 2131 \\
\hline S. siamea (Lam.) H.S. Irwin \& Barn. & Minjiri & Tree, $1 ; \mathrm{pl}$ & $\mathrm{Fl}, \mathrm{Wl}$ & As, De, Sh, Ta & Fw, O & SAK 2132 \\
\hline S. sophera (L.) Roxb. & Kalkeshunda & Shrub; w & $\mathrm{Fl}, \mathrm{Sj}, \mathrm{Rs}$ & $\mathrm{Ka}, \mathrm{Ta}$ & M & SAK 2133 \\
\hline S. tora $($ L.) Roxb. & Kalkeshunda & Herb, er; w & $\mathrm{Fl}, \mathrm{Rs}$ & All upazilas ${ }^{+}$ & M & SAK 2134 \\
\hline Tamarindus indica $\mathrm{L}$. & Tetul & Tree, $1 ; \mathrm{w}$ & Hs, Wl & All upazilas ${ }^{+}$ & $\mathrm{Fr}, \mathrm{T}$ & SAK 2135 \\
\hline \multicolumn{7}{|l|}{ Fabaceae Lindl. } \\
\hline Abrus precatorius $\mathrm{L}$. & Kunch & Herb, cl; w & $\mathrm{Sj}$ & Ka & M & GMH 5274 \\
\hline Butea monosperma (Lam.) Taub. & Palash & Tree, $\mathrm{m} ; \mathrm{pl}$ & Rs, Wl & Ss & $\mathrm{M}, \mathrm{O}$ & GMH 5275 \\
\hline Cajanus cajan (L.) Millsp. & Arhar & Shrub; cv & Af, Fl, Hs & De, Ko, Ta & $\mathrm{M}, \mathrm{Pu}$ & GMH 5276 \\
\hline C. scarabaeoides (L.) Thouars & Banurkali & Herb, cl; w & $\mathrm{Sj}$ & $\mathrm{As}, \mathrm{Ka}, \mathrm{Ss}, \mathrm{Ta}$ & $\mathrm{Gm}, \mathrm{M}$ & GMH 5277 \\
\hline Clitoria ternatea $\mathrm{L}$. & Aparajita & Herb, cl; w & Hs & $\mathrm{Ka}, \mathrm{Ko}, \mathrm{Ss}$ & $\mathrm{M}, \mathrm{O}$ & GMH 5280 \\
\hline Crotalaria pallida Aiton & Jhunjhuni & Herb, er; w & $\mathrm{Fl}, \mathrm{Rs}$ & $\mathrm{Ka}, \mathrm{Ko}, \mathrm{Sh}$ & $\mathrm{Fb}, \mathrm{M}$ & GMH 5281 \\
\hline $\begin{array}{l}\text { *Dalbergia candenatensis } \\
\text { (Dennst.) Prain }\end{array}$ & Chanda lata & Shrub, li; w & $\mathrm{Rb}, \mathrm{Wl}$ & $\mathrm{Sh}^{+}$ & M & GMH 5278 \\
\hline D. sissoo DC. & Sisoo & Tree, $1 ; \mathrm{pl}$ & Rs, Wl & All upazilas ${ }^{+}$ & $\mathrm{T}$ & GMH 5282 \\
\hline *D. spinosa $\mathrm{Roxb}$. & Kutum kanta & Shrub, sc; w & $\mathrm{Rb}, \mathrm{Wl}$ & $\mathrm{Sh}^{+}$ & M & GMH 5283 \\
\hline *Derris scandens (Roxb.) Benth. & Mohajoni lata & Shrub, li; w & Wl & $\mathrm{Sh}^{+}$ & M & GMH 5279 \\
\hline *D. trifoliata Lour. & Kalia lata & Herb, cl; w & $\mathrm{Wl}$ & $\mathrm{Sh}^{+}$ & $\mathrm{Fb}, \mathrm{M}$ & GMH 5284 \\
\hline Desmodium gangeticum (L.) DC. & Salpani & Shrub; w & $\mathrm{Fl}, \mathrm{Sj}, \mathrm{Wl}$ & All upazilas & $\mathrm{Fb}, \mathrm{M}$ & GMH 5289 \\
\hline D. heterophyllum (Willd.) DC. & Bonmotorshuti & Herb, pr; w & $\mathrm{Fl}, \mathrm{Gl}$ & $\mathrm{De}, \mathrm{Ko}, \mathrm{Sh}, \mathrm{Ta}$ & $\mathrm{Lf}, \mathrm{M}$ & GMH 5285 \\
\hline D. laxiflorum DC. & Laximodi & Shrub; w & $\mathrm{Fl}, \mathrm{Rs}$ & $\mathrm{Sh}, \mathrm{Ss}$ & M & GMH 5290 \\
\hline Erythrina fusca Lour. & Kanta mandar & Tree, s; pl & Ml, Rs & As, De, Ka & $\mathrm{M}, \mathrm{O}$ & GMH 5286 \\
\hline E. variegata $\mathrm{L}$. & Parijat & Tree, s; pl & $\mathrm{Fl}, \mathrm{Ml}, \mathrm{Rs}$ & $\mathrm{Ka}, \mathrm{De}, \mathrm{Sh}$ & $\mathrm{M}, \mathrm{O}$ & GMH 5287 \\
\hline $\begin{array}{l}\text { Grona triflora (L.) H. Ohashi \& K. } \\
\text { Ohashi }\end{array}$ & Kulalia & Herb, pr; w & Af, Fl, Gl & All upazilas ${ }^{+}$ & $\mathrm{Gm}, \mathrm{M}$ & MSR 3305 \\
\hline *Mucuna gigantea (Willd.) DC. & Bara alkushi & Herb, cl; w & & $\mathrm{Sh}^{+}$ & M & GMH 5288 \\
\hline M. pruriens (L.) DC. & Bichuti lata & Herb, cl; w & $\mathrm{Sj}, \mathrm{Wl}$ & Ka & M & GMH 5291 \\
\hline Lablab purpureus (L.) Sweet & Shim & Herb, cl; cv & Af, $\mathrm{Hs}$ & All upazilas ${ }^{+}$ & $\mathrm{Pu}, \mathrm{Vg}$ & MSR 3309 \\
\hline Lathyrus sativus L. & Khesari & Herb, cl; cv & Af & De, Ko, Ta & $\mathrm{Lf}, \mathrm{Pu}$ & GMH 5298 \\
\hline Lens culinaris Medik. & Moshur & Herb, pr; cv & Af & $\mathrm{De}, \mathrm{Ka}, \mathrm{Ko}, \mathrm{Ta}$ & $\mathrm{Lf}, \mathrm{Pu}$ & GMH 5299 \\
\hline Pachyrhizus erosus (L.) Urb. & Shakalu & Herb, cl; cv & Hs, Rs & $\mathrm{Sh}, \mathrm{Ss}$ & $\mathrm{M}, \mathrm{Vg}$ & GMH 5296 \\
\hline *Pongamia pinnata (L.) Pierre & Koroch & Tree, $\mathrm{m} ; \mathrm{w}$ & $\mathrm{Rb}$ & Sh & $\mathrm{M}, \mathrm{Fw}$ & MSR 3318 \\
\hline Sesbania cannabina (Retz.) Pers. & Dhonchi & Shrub; cv & Af, Fl, Ml & All upazilas & $\mathrm{Fb}, \mathrm{Gm}$ & GMH 5297 \\
\hline Uraria lagopodioides (L.) Desv. & Chakulia & Shrub; w & $\mathrm{Sj}, \mathrm{Wl}$ & Ko & & GMH 5292 \\
\hline Vicia hirsuta (L.) S.F. Gray & Masurchana & Herb, pr; w & Af & Ko, Ta & Lf, Gm & GMH 5293 \\
\hline Vigna marina (Burm.) Merr. & Nona shim & Herb, cl; w & $\mathrm{Fm}, \mathrm{Rb}$ & $\mathrm{Ka}, \mathrm{Sh}+$ & Lf, M & MSR 3315 \\
\hline V. mungo (L.) Hepper & Mashkalai & Herb, pr; cv & Af, Fl, Rs & De, Ko, Ta & $\mathrm{Gm}, \mathrm{Pu}$ & GMH 5294 \\
\hline V. trilobata (L.) Verdc. & Jangli moong & Herb, cl; w & $\mathrm{Fl}, \mathrm{Gl}$ & Ss & $\mathrm{Gm}, \mathrm{Lf}$ & GMH 5295 \\
\hline
\end{tabular}


Table 1 contd.

\begin{tabular}{|c|c|c|c|c|c|c|}
\hline Scientific name & Bangla name & Habit & Habitat & Distribution & Use & RSE \\
\hline $\begin{array}{l}\text { V. unguiculata (L.) Walp. } \\
\text { Haloragaceae R. Br. }\end{array}$ & Borboti & Herb, cl; cv & Af, Hs & $\mathrm{Ka}, \mathrm{Ko}, \mathrm{Ss}$ & $\mathrm{Pu}, \mathrm{Vg}$ & GMH 5300 \\
\hline $\begin{array}{l}\text { Myriophyllum tuberculatum Roxb. } \\
\text { Lythraceae J. St.-Hil. }\end{array}$ & Kulabahupatri & Herb, sm; w & Wtl & Sh, Ta & M & MAR 2983 \\
\hline Ammannia baccifera $\mathrm{L}$. & Dadmari & Herb, er; w & Af, Fl, Wtl & $\mathrm{Ka}, \mathrm{Sh}^{+}, \mathrm{Ss}$ & M & GMH 5301 \\
\hline A. multiflora Roxb. & Acidpatta & Herb, er; w & Af, Fl, Wtl & Sh, Ss, Ta & M & GMH 5302 \\
\hline Lagerstroemia indica $\mathrm{L}$. & Jarul & Tree, s; pl & Rs & As, Ss & M. O & GMH 5303 \\
\hline L. speciosa (L.) Pers. & Jarul & Tree, $1 ; \mathrm{pl}$ & Rs, Wl & As, Ss & $\mathrm{M}, \mathrm{O}$ & GMH 5304 \\
\hline Lawsonia inermis L. & Mehedi & Tree, s; pl & $\mathrm{Hs}$ & All upazilas ${ }^{+}$ & Dy, M & MSR 3311 \\
\hline Punica granatum $\mathrm{L}$. & Dalim, Bedana & Shrub; pl & Hs & $\mathrm{Ka}, \mathrm{Ko}, \mathrm{Sh}, \mathrm{Ss}$ & Dy, Fr & KMNI 062 \\
\hline *Sonneratia apetala Buch.-Ham. & Kewra & Tree, $1 ; \mathrm{w}$ & $\mathrm{Rb}, \mathrm{Wl}$ & $\mathrm{De}, \mathrm{Ka}, \mathrm{Sh}^{+}$ & Fr, M & GMH 5305 \\
\hline Trapa incisa Siebold \& Zucc. & Paniphal & Herb, fr; w & Wtl & $\mathrm{De}, \mathrm{Ka}, \mathrm{Ko}, \mathrm{Sh}$ & Fr, M & GMH 5306 \\
\hline $\begin{array}{l}\text { T. natans L. } \\
\text { Myrtaceae Juss. }\end{array}$ & Shingra & Herb, fr; w & Wtl & $\mathrm{De}, \mathrm{Ko}, \mathrm{Sh}$ & Fr, M & GMH 5307 \\
\hline Callistemon citrinus (Curtis) Skeels & Bottlebrush & Tree, s; pl & Hs, Rs & As, Ko, Sh, Ss & $\mathrm{O}$ & MSR 3317 \\
\hline Eucalyptus camaldulensis Dehnh. & Eucalyptus & Tree, $1 ; \mathrm{pl}$ & Rs, Wl & All upazilas ${ }^{+}$ & $\mathrm{M}, \mathrm{T}$ & MAR 2984 \\
\hline Psidium guajava $\mathrm{L}$. & Peyara & Tree, s; pl & Hs & All upazilas ${ }^{+}$ & Fr, M & KMNI 011 \\
\hline Syzygium cumini (L.) Skeels & Kalojam & Tree, $1 ; \mathrm{pl}$ & Hs, Rs, Wl & $\mathrm{Ka}, \mathrm{Ko}, \mathrm{Sh}, \mathrm{Ss}$ & Fr, T & KMNI 012 \\
\hline S. fruticosum DC. & Khudi jam & Tree, $\mathrm{m} ; \mathrm{w}$ & Ml, Wl & $\mathrm{Ka}, \mathrm{Ko}, \mathrm{Sh}^{+}$ & Fr, T & MSR 3312 \\
\hline Onagraceae Juss. & & & & & & \\
\hline Ludwigia adscendens (L.) H. Hara & Keshordam & Herb, fr; w & Wtl & All upazilas ${ }^{+}$ & M & GMH 5308 \\
\hline L. hyssopifolia (G. Don) Exell & Panipalong & Herb, er; w & Af, Fl, Wtl & All upazilas ${ }^{+}$ & Dy, M & GMH 5368 \\
\hline L. octovalvis (Jacq.) P.H. Raven & Bon labonga & Herb, er; w & Ml, Wtl & $\mathrm{Ta}$ & M & GMH 5369 \\
\hline L. perennis $\mathrm{L}$. & Amorkura & Herb, er; w & $\mathrm{Fl}, \mathrm{Gl}$ & Ko, Sh, Ta & M & MAR 2985 \\
\hline Combretaceae R. Br. & & & & & & \\
\hline Combretum indicum (L.) DeFilipps & Madhuri lata & Shrub, li; pl & Hs & Ss & M, O & MAR 2986 \\
\hline *Lumnitzera racemosa Willd. & Kirpa & Tree, s; w & Fm, Wl & $\mathrm{Sh}^{+}$ & Dy, Fw & GMH 5309 \\
\hline $\begin{array}{l}\text { Terminalia arjuna (Roxb. ex DC.) } \\
\text { Wight \& Arn. }\end{array}$ & Arjun & Tree, $1 ; \mathrm{pl}$ & Rs & All upazilas ${ }^{+}$ & M & MSR 3316 \\
\hline T. bellirica (Gaertn.) Roxb. & Bohera & Tree, $1 ; \mathrm{pl}$ & Rs, Wl & All upazilas ${ }^{+}$ & M & MSR 3303 \\
\hline T. catappa $\mathrm{L}$. & Kathbadam & Tree, $1 ; \mathrm{pl}$ & Rs & All upazilas ${ }^{+}$ & $\mathrm{M}, \mathrm{Nu}$ & MSR 3314 \\
\hline T. chebula Retz. & Horitoki & Tree, $1 ; \mathrm{pl}$ & Rs, Wl & All upazilas ${ }^{+}$ & M & MSR 3304 \\
\hline Rhizophoraceae Pers. & & & & & & \\
\hline *Bruguiera gymnorhiza (L.) Lam. & Lal kakra & Tree, $1 ; \mathrm{w}$ & Wl & $\mathrm{Sh}^{+}$ & Dy, $\mathrm{T}$ & GMH 5310 \\
\hline *B. sexangula (Lour.) Poir. & Shobuj kakra & Tree, $1 ; \mathrm{w}$ & $\mathrm{Wl}$ & $\mathrm{Sh}^{+}$ & Dy, $\mathrm{T}$ & GMH 5311 \\
\hline $\begin{array}{l}\text { *Ceriops decandra (Griff.) W. } \\
\text { Theob. }\end{array}$ & Goran & Tree, s; w & $\mathrm{Wl}$ & $\mathrm{Sh}^{+}$ & Dy, Fw & GMH 5312 \\
\hline *Kandelia candel (L.) Druce & Bhatkathi & Tree, s; w & $\mathrm{Rb}, \mathrm{Fm}$ & $\mathrm{Sh}^{+}$ & Dy, Fw & GMH 5313 \\
\hline *Rhizophora apiculata Blume & Jhana & Tree, $\mathrm{m} ; \mathrm{w}$ & $\mathrm{Rb}, \mathrm{Fm}$ & $\mathrm{Sh}^{+}$ & Fw, M & GMH 5314 \\
\hline $\begin{array}{l}\text { *R. mucronata Lam. } \\
\text { Cornaceae Bercht. ex J. Presl }\end{array}$ & Jhana & Tree, $1 ; \mathrm{w}$ & $\mathrm{Rb}, \mathrm{Fm}$ & $\mathrm{Sh}^{+}$ & Dy, Fw & GMH 5315 \\
\hline $\begin{array}{l}\text { Alangium salviifolium (L. f.) } \\
\text { Wangerin }\end{array}$ & Aikha & Tree, $\mathrm{m} ; \mathrm{w}$ & $\mathrm{Sj}, \mathrm{Wl}$ & $\mathrm{De}, \mathrm{Ka}$ & $\mathrm{M}, \mathrm{T}$ & MAR 2987 \\
\hline Loranthaceae Juss. & & & & & & \\
\hline Dendrophthoe falcata (L. f.) Etting. & Bajrangi & Shrub, ps; w & Op & $\mathrm{De}, \mathrm{Ka}, \mathrm{Sh}^{+}$ & M & MSR 3306 \\
\hline $\begin{array}{l}\text { Macrosolen cochinchinensis } \\
\text { (Lour.) van Tiegh. }\end{array}$ & Renda & Shrub, ps; w & Op & $\mathrm{De}, \mathrm{Ka}, \mathrm{Sh}^{+}$ & M & MSR 3301 \\
\hline Scurrula parasitica $\mathrm{L}$. & Porgacha & Shrub, ps; w & Op & $\mathrm{Ka}, \mathrm{Sh}^{+}$, & M & MSR 3307 \\
\hline Viscum monoicum Roxb. ex DC. & Bhanda & Herb, ps; w & Op & $\mathrm{Sh}^{+}$ & M & MSR 3330 \\
\hline
\end{tabular}


Table 1 contd.

\begin{tabular}{|c|c|c|c|c|c|c|}
\hline Scientific name & Bangla name & Habit & Habitat & Distribution & Use & RSE \\
\hline \multicolumn{7}{|l|}{ Celastraceae R. Br. } \\
\hline *Salacia chinensis $\mathrm{L}$. & Choit boroi & Shrub, sc; w & Fm, Wl & $\mathrm{Sh}^{+}$ & Fr, M & GMH 5316 \\
\hline \multicolumn{7}{|l|}{ Euphorbiaceae Juss. } \\
\hline Acalypha ciliata Forssk. & Unknown & Herb, er; w & $\mathrm{Fl}, \mathrm{Sj}$ & Ka, Ko, Ta & M & MAR 2988 \\
\hline A. indica $\mathrm{L}$. & Muktajhuri & Herb, er; w & $\mathrm{Fl}, \mathrm{Gl}, \mathrm{Rs}$ & $\mathrm{Ka}, \mathrm{Ko}, \mathrm{Ta}, \mathrm{Ss}$ & M & MAR 2989 \\
\hline Baliospermum calycinum Müll. Arg. & Danti & Shrub; w & $\mathrm{Sj}, \mathrm{Wl}$ & $\mathrm{De}$ & M & MAR 2990 \\
\hline B. solanifolium (Burm.) Suresh & Donti & Shrub; w & $\mathrm{Sj}, \mathrm{Wl}$ & De & M & MAR 2991 \\
\hline $\begin{array}{l}\text { Chrozophora rottleri (Geiseler) A. } \\
\text { Juss. ex Spreng. }\end{array}$ & Khudiphora & Herb, er; w & & As, Ko, Sh, Ta & M & MAR 2993 \\
\hline $\begin{array}{l}\text { Codiaeum variegatum (L.) Rumph. } \\
\text { ex A. Juss. }\end{array}$ & Batabahar & Shrub; pl & Hs, Ml & $\mathrm{De}, \mathrm{Ka}, \mathrm{Ss}$ & M, O & MAR 2994 \\
\hline Croton bonplandianus Baill. & Bandhone & Herb, er; w & Af, Fl, Rs & All upazilas & M & GMH 5317 \\
\hline Euphorbia antiquorum L. & Tiktasij & Shrub; w & Hs, Ml & As, $\mathrm{Sh}$ & $\mathrm{M}, \mathrm{O}$ & GMH 5318 \\
\hline E. hirta $\mathrm{L}$. & Baradudhia & Herb, pr; w & $\mathrm{Fl}, \mathrm{Gl}, \mathrm{Rs}$ & All upazilas ${ }^{+}$ & M & GMH 5319 \\
\hline E. neriifolia $\mathrm{L}$. & Manosha sij & Shrub; pl & Hs, Ml & As, $\mathrm{Sh}$ & $\mathrm{M}, \mathrm{O}$ & GMH 5320 \\
\hline E. thymifolia $\mathrm{L}$. & Swetkerui & Herb, pr; w & $\mathrm{Fl}, \mathrm{Gl}, \mathrm{Rs}$ & All upazilas ${ }^{+}$ & M & GMH 5321 \\
\hline E. tirucalli L. & Narasaji & Shrub; pl & Hs, Rs & $\mathrm{Sh}, \mathrm{Ss}$ & $\mathrm{M}, \mathrm{O}$ & GMH 5322 \\
\hline E. tithymaloides $\mathrm{L}$. & Bera chita & Herb, er; w & Ml, Rs & $\mathrm{De}, \mathrm{Ka}, \mathrm{Sh}$ & $\mathrm{He}, \mathrm{M}$ & GMH 5323 \\
\hline *Excoecaria agallocha $\mathrm{L}$. & Gewa & Tree, $1 ; \mathrm{w}$ & $\mathrm{Wl}$ & $\mathrm{Sh}^{+}$ & $\mathrm{M}, \mathrm{Pp}$ & GMH 5324 \\
\hline $\begin{array}{l}\text { Flueggea virosa (Roxb. ex Willd.) } \\
\text { Royle }\end{array}$ & Khaukra & Shrub; w & $\mathrm{Sj}, \mathrm{Wl}$ & $\mathrm{De}, \mathrm{Ka}, \mathrm{Ko}$ & M & MAR 3118 \\
\hline Jatropha curcas L. & Bherenda & Shrub; pl & Rs & As, Ka, Ta & $\mathrm{He}, \mathrm{M}$ & GMH 5326 \\
\hline J. gossypiifolia $\mathrm{L}$. & Lalbherenda & Shrub; w & $\mathrm{Fl}$, Rs & As, Ka, Ko, Ta & $\mathrm{He}, \mathrm{M}$ & GMH 5327 \\
\hline Mallotus nudiflora $\mathrm{L}$. & Latim, Petali & Tree, $1 ; \mathrm{w}$ & $\mathrm{Fl}, \mathrm{Ml}$ & $\mathrm{Ka}, \mathrm{Ta}$ & $\mathrm{M}, \mathrm{T}$ & GMH 5336 \\
\hline M. repandus (Willd.) Müll. Arg. & Gunti, Jhanti & Shrub, sc; w & $\mathrm{Sj}, \mathrm{Wl}$ & Ko, Ta & $\mathrm{M}$ & GMH 5328 \\
\hline Ricinus communis $\mathrm{L}$. & Bherenda & Shrub; w & $\mathrm{Fl}, \mathrm{Hs}$ & All upazilas & $\mathrm{M}, \mathrm{Oy}$ & GMH 5334 \\
\hline *Shirakiopsis indica (Willd.) Esser & Hurmui & Tree, s; w & $\mathrm{Fm}, \mathrm{Wl}$ & $\mathrm{Sh}^{+}$ & $\mathrm{M}, \mathrm{Fp}$ & GMH 5335 \\
\hline Suregada multiflora (A. Juss.) Baill. & Ban naringa & Tree, s; w & $\mathrm{Wl}$ & $\mathrm{Ka}$ & Fw, M & KMNI 013 \\
\hline Tragia involucrata $\mathrm{L}$. & Chotrapatta & Herb, cl; w & $\mathrm{Sj}$ & $\mathrm{Ka}, \mathrm{Ta}$ & M & KMNI 014 \\
\hline \multicolumn{7}{|l|}{ Phyllanthaceae Martinov } \\
\hline Breynia vitis-idaea (Burm. f.) Fisch. & Vitasalpoti & Tree, s; w & $\mathrm{Sj}, \mathrm{Wl}$ & $\mathrm{De}, \mathrm{Ka}$ & M & MAR 2992 \\
\hline Phyllanthus acidus (L.) Skeels & Arboroi & Tree, s; pl & Hs & As, Ko, Sh, Ss & Fr, M & GMH 5329 \\
\hline P. emblica L. & Amloki & Tree, s; pl & Hs, Rs & All upazilas ${ }^{+}$ & Fr, M & GMH 5330 \\
\hline P. niruri $\mathrm{L}$. & Bhuiamla & Herb, er; w & Af, Fl, Gl & All upazilas ${ }^{+}$ & Dy, M & GMH 5331 \\
\hline P. reticulatus Poir. & Chitki & Shrub; w & $\mathrm{Fl}, \mathrm{Sj}$ & $\mathrm{De}, \mathrm{Ka}, \mathrm{Ko}, \mathrm{Ta}$ & Dy, M & GMH 5332 \\
\hline P. urinaria $\mathrm{L}$. & Kalochitki & Herb, er; w & $\mathrm{Fl}, \mathrm{Gl}$ & $\mathrm{Ta}$ & M & GMH 5333 \\
\hline \multicolumn{7}{|l|}{ Rhamnaceae Juss. } \\
\hline Gouania tiliifolia Lam. & Harjen gota & Shrub, sc; w & $\mathrm{Sj}, \mathrm{Wl}$ & $\mathrm{De}, \mathrm{Ka}$ & $\mathrm{Co}, \mathrm{M}$ & MAR 2995 \\
\hline $\begin{array}{l}\text { Sarcomphalus mauritianus (Lam.) } \\
\text { Raf. }\end{array}$ & Boroi & Tree, m; w & Hs, Wl & All upazilas ${ }^{+}$ & Fr, M & MAR 2996 \\
\hline $\begin{array}{l}\text { Ziziphus oenoplia (L.) Mill. } \\
\text { Leeaceae Dumort. }\end{array}$ & Bonboroi & Shrub, sc; w & $\mathrm{Sj}, \mathrm{Wl}$ & $\mathrm{De}, \mathrm{Ko}, \mathrm{Ta}$ & $\mathrm{He}, \mathrm{M}$ & MAR 2997 \\
\hline Leea aequata $\mathrm{L}$. & Kukur jihwa & Shrub; w & $\mathrm{Sj}, \mathrm{Wl}$ & $\mathrm{De}, \mathrm{Ka}$ & M & MAR 2998 \\
\hline $\begin{array}{l}\text { L. indica (Burm. f.) Merr. } \\
\text { Vitaceae Juss. }\end{array}$ & Kurkur & Shrub; w & $\mathrm{Sj}, \mathrm{Wl}$ & $\mathrm{De}, \mathrm{Ka}, \mathrm{Ko}, \mathrm{Ta}$ & $\mathrm{Gm}, \mathrm{M}$ & MAR 2999 \\
\hline $\begin{array}{l}\text { Ampelocissus barbata (Wall.) } \\
\text { Planch. }\end{array}$ & Jharila & Herb, cl; w & $\mathrm{Sj}, \mathrm{Wl}$ & $\mathrm{De}, \mathrm{Ka}, \mathrm{Ss}$ & M & MAR 3000 \\
\hline A. latifolia (Roxb.) Planch. & Gowalia lata & Herb, cl; w & $\mathrm{Wl}$ & De, Ka, Ko & M & MAR 3001 \\
\hline $\begin{array}{l}\text { Causonis trifolia (L.) Mabb. \& J. } \\
\text { Wen }\end{array}$ & Angur lata & Herb, cl; w & $\mathrm{Sj}, \mathrm{Wl}$ & All upazilas ${ }^{+}$ & Lf, M & KMNI 017 \\
\hline
\end{tabular}


Table 1 contd.

\begin{tabular}{|c|c|c|c|c|c|c|}
\hline Scientific name & Bangla name & Habit & Habitat & Distribution & Use & RSE \\
\hline Cissus adnata Roxb. & Bhatia lata & Herb, cl; w & $\mathrm{Sj}, \mathrm{Wl}$ & $\mathrm{Ka}, \mathrm{Ss}$ & M & KMNI 018 \\
\hline C. quadrangularis $\mathrm{L}$. & Harjora & Herb, cl; w & Hs, Rs & $\mathrm{De}, \mathrm{Ka}$ & M & KMNI 019 \\
\hline $\begin{array}{l}\text { Tetrastigma angustifolium (Roxb.) } \\
\text { Planch. }\end{array}$ & Nekungriubi & Herb, cl; w & $\mathrm{Sj}, \mathrm{Wl}$ & $\mathrm{De}, \mathrm{Ka}, \mathrm{Ko}, \mathrm{Ss}$ & M & MAR 3002 \\
\hline $\begin{array}{l}\text { T. leucostaphylum (Dennst.) Alston } \\
\text { Sapindaceae Juss. }\end{array}$ & Horina lata & Herb, cl; w & $\mathrm{Sj}, \mathrm{Wl}$ & $\mathrm{De}, \mathrm{Ka}, \mathrm{Ss}$ & M & MAR 3003 \\
\hline Allophylus cobbe (L.) Raeusch. & Rakhal chita & Shrub; w & $\mathrm{Sj}, \mathrm{Wl}$ & $\mathrm{Ka}$ & Fw, M & GMH 5337 \\
\hline Cardiospermum halicacabum $\mathrm{L}$. & Lataphutki & Herb, cl; w & $\mathrm{Fl}, \mathrm{Sj}$ & Ko, Sh, Ss, Ta & $\mathrm{M}, \mathrm{Vg}$ & GMH 5338 \\
\hline Dimocarpus longan Lour. & Ashphal & Tree, $\mathrm{m} ; \mathrm{pl}$ & Hs & $\mathrm{Ka}$ & $\mathrm{Fr}$ & MAR 3119 \\
\hline *Dodonaea viscosa Jacq. & Pani phul & Tree, s; w & Fm, Wl & $\mathrm{Sh}^{+}$ & Fw, M & GMH 5471 \\
\hline $\begin{array}{l}\text { Lepisanthes rubiginosa (Roxb.) } \\
\text { Leenh. }\end{array}$ & Horina & Tree, s; w & $\mathrm{Fl}, \mathrm{Wl}$ & $\mathrm{De}, \mathrm{Ka}, \mathrm{Ko}$ & $\mathrm{Fr}, \mathrm{Fw}$ & GMH 5340 \\
\hline \multicolumn{6}{|l|}{ Burseraceae Kunth } & GMH 5341 \\
\hline $\begin{array}{l}\text { Garuga pinnata Roxb. } \\
\text { Anacardiaceae } \mathrm{R} . \mathrm{Br} \text {. }\end{array}$ & Kapila & Tree, $1 ; \mathrm{w}$ & $\mathrm{Wl}$ & $\mathrm{De}$ & Fr, T & MAR 3004 \\
\hline $\begin{array}{l}\text { Lannea coromandelica (Houtt.) } \\
\text { Merr. }\end{array}$ & Jiga & Tree, s; w & $\mathrm{Ml}, \mathrm{Rs}$ & All upazilas ${ }^{+}$ & $\mathrm{He}, \mathrm{Gu}$ & MAR 3005 \\
\hline Mangifera indica $\mathrm{L}$. & Aam & Tree, 1 ; w & $\mathrm{Hs}, \mathrm{Wl}$ & All upazilas ${ }^{+}$ & $\mathrm{Fr}, \mathrm{T}$ & MAR 3006 \\
\hline Spondias dulcis Parkinson & Amrah & Tree, $1 ; \mathrm{pl}$ & Hs & All upazilas ${ }^{+}$ & $\mathrm{Fr}$ & MAR 3007 \\
\hline $\begin{array}{l}\text { S. pinnata (L. f.) Kurz } \\
\text { Meliaceae Juss. }\end{array}$ & Bon amrah & Tree, $1 ; \mathrm{pl}$ & Wl & $\mathrm{De}, \mathrm{Ka}$ & $\mathrm{Fr}$ & GMH 5467 \\
\hline *Aglaia cucullata (Roxb.) Pellegr. & Amoor & Tree, s; w & $\mathrm{Rb}, \mathrm{Wl}$ & $\mathrm{Sh}^{+}$ & $\mathrm{M}, \mathrm{T}$ & MSR 3329 \\
\hline $\begin{array}{l}\text { Aphanamixis polystachya (Wall.) } \\
\text { R. Parker }\end{array}$ & Pithraj & Tree, m; w & Hs, Wl & $\mathrm{De}, \mathrm{Ka}, \mathrm{Ko}, \mathrm{Ta}$ & $\mathrm{M}, \mathrm{Oy}$ & GMH 5342 \\
\hline Azadirachta indica A. Juss. & Neem & Tree, m; w & Rs, Wl & All upazilas ${ }^{+}$ & $\mathrm{M}, \mathrm{T}$ & GMH 5343 \\
\hline Khaya anthotheca (Welw.) C. DC. & Lombu & Tree, $1 ; \mathrm{pl}$ & Rs & All upazilas & $\mathrm{T}$ & GMH 5344 \\
\hline Melia azedarach $\mathrm{L}$. & Ghora neem & Tree, $\mathrm{m} ; \mathrm{pl}$ & Ml, Rs & All upazilas ${ }^{+}$ & $\mathrm{M}, \mathrm{T}$ & GMH 5345 \\
\hline Swietenia macrophylla King & Bara mehagani & Tree, $1 ; \mathrm{pl}$ & Hs, Ml, Rs & All upazilas ${ }^{+}$ & $\mathrm{T}$ & GMH 5346 \\
\hline S. mahagoni (L.) Jacq. & Mehagani & Tree, $1 ; \mathrm{pl}$ & $\mathrm{Hs}, \mathrm{Rs}$ & As, Ka, Sh, Ss & $\mathrm{T}$ & GMH 5347 \\
\hline Toona ciliata M. Roem. & Toon & Tree, $1 ; \mathrm{w}$ & Rs, Wl & $\mathrm{De}, \mathrm{Ka}$ & Dy, M & GMH 5348 \\
\hline *Xylocarpus granatum J. Koenig & Dhundal & Tree, m; w & $\mathrm{Rb}, \mathrm{Wl}$ & $\mathrm{Sh}^{+}$ & $\mathrm{M}, \mathrm{T}$ & MSR 3322 \\
\hline $\begin{array}{l}\text { *X. moluccensis (Lam.) M. Roem. } \\
\text { Rutaceae Juss. }\end{array}$ & Poshur & Tree, m; w & $\mathrm{Wl}$ & $\mathrm{Sh}^{+}$ & $\mathrm{M}, \mathrm{T}$ & MSR 3328 \\
\hline \multicolumn{7}{|l|}{ Rutaceae Juss. } \\
\hline Aegle marmelos (L.) Corrêa & Bel & Tree, m; w & Hs, Ml, Wl & All upazilas ${ }^{+}$ & Fr, M & KMNI 020 \\
\hline $\begin{array}{l}\text { Citrus aurantiifolia (Christm.) } \\
\text { Swingle }\end{array}$ & Lebu & Shrub; pl & Hs & All upazilas & $\mathrm{Fr}$ & MAR 3008 \\
\hline C. maxima (Burm.) Merr. & Jambura & Tree, s; pl & $\mathrm{Ml}, \mathrm{Hs}$ & All upazilas ${ }^{+}$ & $\mathrm{Fr}$ & MAR 3009 \\
\hline Feronia limonia (L.) Swingle & Kadbel & Tree, $\mathrm{m} ; \mathrm{pl}$ & $\mathrm{Ml}, \mathrm{Hs}$ & All upazilas ${ }^{+}$ & $\mathrm{Fr}$ & KMNI 021 \\
\hline $\begin{array}{l}\text { Glycosmis pentaphylla (Retz.) A. } \\
\text { DC. }\end{array}$ & Datmajoni & Shrub; w & $\mathrm{Fl}, \mathrm{Sj}, \mathrm{Wl}$ & All upazilas & M & KMNI 022 \\
\hline Murraya koenigii (L.) Spreng. & Curry patta & Tree, s; w & $\mathrm{Fl}, \mathrm{Wl}$ & $\mathrm{Ka}, \mathrm{Ta}$ & $\mathrm{M}, \mathrm{Sp}$ & KMNI 023 \\
\hline M. paniculata (L.) Jack & Kamini & Tree, $\mathrm{s} ; \mathrm{pl}$ & Rs, Wl & Ss & $\mathrm{M}, \mathrm{O}$ & KMNI 024 \\
\hline \multicolumn{7}{|l|}{ Oxalidaceae R. Br. } \\
\hline Averrhoa bilimbi L. & Bilimbi & Tree, s; pl & Hs & As, Ko, Sh, Ss & Fr, M & MAR 3011 \\
\hline A. carambola $\mathrm{L}$. & Kamranga & Tree, s; pl & Hs & As, De, Sh, Ss & Fr, M & MAR 3012 \\
\hline Oxalis corniculata $\mathrm{L}$. & Amrul & Herb, pr; w & Af, Gl, Rs & All upazilas & $\mathrm{M}, \mathrm{Vg}$ & MAR 3013 \\
\hline
\end{tabular}


Table 1 contd.

\begin{tabular}{|c|c|c|c|c|c|c|}
\hline Scientific name & Bangla name & Habit & Habitat & Distribution & Use & RSE \\
\hline \multicolumn{7}{|l|}{ Balsaminaceae A. Rich. } \\
\hline Impatiens balsamina $\mathrm{L}$. & Dopati & Herb, er; pl & Hs, Ml & As, De, Ko, Ss & $\mathrm{M}, \mathrm{O}$ & KMNI 063 \\
\hline \multicolumn{7}{|l|}{ Apiaceae Lindl. } \\
\hline Centella asiatica (L.) Urb. & Thankuni & Herb, cr; w & Af, Fl, Ml & All upazilas ${ }^{+}$ & M & GMH 5349 \\
\hline Coriandrum sativum $\mathrm{L}$. & Dhonia & Herb, er; cv & Af, Fl, Hs, & Ko & $\mathrm{M}, \mathrm{Sp}$ & GMH 5350 \\
\hline Eryngium foetidum $\mathrm{L}$. & Bilatedhoneya & Herb, er; w & Af, Hs & As, $\mathrm{De}, \mathrm{Sh}^{+}, \mathrm{Ss}$ & $\mathrm{M}, \mathrm{Sp}$ & GMH 5351 \\
\hline $\begin{array}{l}\text { Oenanthe benghalensis Benth. \& } \\
\text { Hook. f. }\end{array}$ & Bon-dhonia & Herb, er; w & $\mathrm{Fl}, \mathrm{Gl}, \mathrm{Ml}$ & As, Ka, Ta & $\mathrm{M}$ & GMH 5352 \\
\hline \multicolumn{7}{|l|}{ Apocynaceae Juss. } \\
\hline Allamanda cathartica $\mathrm{L}$. & Ghonta phul & Shrub; pl & Hs, Rs & As, Ss & $\mathrm{O}$ & MAR 3014 \\
\hline Alstonia scholaris (L.) R. Br. & Chhatim & Tree, $1 ; \mathrm{w}$ & Rs, W1 & $\mathrm{Ka}, \mathrm{Ta}$ & $\mathrm{M}, \mathrm{T}$ & MAR 3015 \\
\hline Calotropis gigantea (L.) Dryand. & Akondo & Shrub; w & $\mathrm{Ml}, \mathrm{Rs}$ & As, De, Ka, Ta & $\mathrm{Fb}, \mathrm{M}$ & KMNI 025 \\
\hline C. procera (Aiton) Dryand. & Shda akondo & Shrub; w & Rs & As, $\mathrm{Ta}$ & $\mathrm{Fb}, \mathrm{M}$ & $K M N I 026$ \\
\hline Carissa carandas $\mathrm{L}$. & Karamcha & Shrub; pl & $\mathrm{Hs}, \mathrm{Sj}$ & Ss & Fr & $M A R 3016$ \\
\hline Cascabela thevetia (L.) Lippold & Kolkey phul & Tree, s; pl & Hs & $\mathrm{De}, \mathrm{Sh}, \mathrm{Ss}$ & $\mathrm{M}, \mathrm{O}$ & MAR 3017 \\
\hline Catharanthus roseus (L.) G. Don & Noyantara & Herb, er; w & Hs, Rs & As, Sh, Ss, Ta & $\mathrm{M}, \mathrm{O}$ & KMNI 027 \\
\hline *Cerbera odollam Gaertn. & Dahur & Tree, s; w & Fm, Wl & $\mathrm{Sh}^{+}$ & $\mathrm{Fb}, \mathrm{M}$ & GMH 5353 \\
\hline *Ceropegia lucida Wall. & Lucipegia & Herb, cl; w & $\mathrm{Fm}$ & $\mathrm{Sh}^{+}$ & M & MSR 3327 \\
\hline $\begin{array}{l}\text { Dregea volubilis (L. f.) Benth. ex } \\
\text { Hook. f. }\end{array}$ & Jukti phul & Herb, cl; w & Wl & De & $\mathrm{Fb}, \mathrm{M}$ & GMH 5354 \\
\hline *Finlaysonia obovata Wall. & Mamakola & Herb, cl; w & $\mathrm{Rb}, \mathrm{Wl}$ & $\mathrm{Sh}^{+}$ & $\mathrm{Fb}, \mathrm{M}$ & GMH 5355 \\
\hline Hoya lanceolata Wall. ex D. Don & Futki lata & Herb, ps; w & Op; W1 & De & $\mathrm{Fb}, \mathrm{M}$ & GMH 5356 \\
\hline H. parasitica Wall. ex Traill & Futki lata & Herb, ps; w & Op; Wl & $\mathrm{Sh}^{+}$ & $\mathrm{Fb}, \mathrm{M}$ & GMH 5357 \\
\hline $\begin{array}{l}\text { Hemidesmus indicus (L.) R. Br. ex } \\
\text { Schult. }\end{array}$ & Anantomul & Herb, cl; w & $\mathrm{Fl}, \mathrm{Gl}$ & $\mathrm{De}, \mathrm{Ka}, \mathrm{Ta}$ & $\mathrm{Fb}, \mathrm{M}$ & GMH 5358 \\
\hline Ichnocarpus frutescens (L.) Aiton & Parallia lata & Herb, cl; w & $\mathrm{Fl}, \mathrm{Sj}, \mathrm{Wl}$ & $\mathrm{Ka}, \mathrm{Ko}, \mathrm{Ta}$ & $\mathrm{Fb}, \mathrm{M}$ & GMH 5359 \\
\hline Nerium oleander L. & Rakta karobi & Tree, $\mathrm{s} ; \mathrm{pl}$ & Hs, Rs & Ss & $\mathrm{M}, \mathrm{O}$ & GMH 5360 \\
\hline $\begin{array}{l}\text { Pentatropis capensis (L. f.) } \\
\text { Bullock }\end{array}$ & Panchabrti lata & Herb, cl; w & $\mathrm{Sj}, \mathrm{Wl}$ & $\mathrm{Ka}$ & M & GMH 5361 \\
\hline $\begin{array}{l}\text { Pergularia daemia (Forssk.) } \\
\text { Chiov. }\end{array}$ & Chagalbati & Herb, cl; w & $\mathrm{Sj}, \mathrm{Rs}$ & Sh, Ss & $\mathrm{Fb}, \mathrm{M}$ & GMH 5362 \\
\hline $\begin{array}{l}\text { *Parsonsia alboflavescens } \\
\text { (Dennst.) Mabb. }\end{array}$ & Pasonsi & Herb, cl; w & $\mathrm{Fm}, \mathrm{Rb}$ & $\mathrm{Sh}^{+}$ & $\mathrm{Fb}, \mathrm{M}$ & GMH 5363 \\
\hline Plumeria alba $\mathrm{L}$. & Shada kathgolap & Tree, $\mathrm{m} ; \mathrm{pl}$ & Hs, Rs & Ss & $\mathrm{M}, \mathrm{O}$ & GMH 5364 \\
\hline P. rubra L. & Lal kathgolap & Tree, $\mathrm{m} ; \mathrm{pl}$ & Hs, Rs & Ss & $\mathrm{M}, \mathrm{O}$ & GMH 5365 \\
\hline $\begin{array}{l}\text { Rauvolfia serpentina (L.) Benth. ex } \\
\text { Kurz }\end{array}$ & Sarpogondha & Herb, er; w & W1 & De & $\mathrm{M}, \mathrm{O}$ & GMH 5366 \\
\hline *Sarcolobus globosus Wall. & Bawali lata & Herb, cl; w & $\mathrm{Wl}$ & $\mathrm{Sh}^{+}$ & $\mathrm{M}, \mathrm{Vg}$ & GMH 5367 \\
\hline $\begin{array}{l}\text { Tabernaemontana divaricata (L.) } \\
\text { R. Br. ex Roem \& Schult. }\end{array}$ & Tagar & Shrub; w & $\mathrm{Rs}, \mathrm{Sj}, \mathrm{Wl}$ & De, Ko, Ta & $\mathrm{M}, \mathrm{O}$ & GMH 5368 \\
\hline Telosma cordata (Burm. f.) Merr. & Kanja lata & Herb, cl; w & $\mathrm{Sj}, \mathrm{Wl}$ & De & M & GMH 5370 \\
\hline *Tylophora indica (Burm. f.) Merr. & Antamul & Herb, cl; w & $\mathrm{Fm}, \mathrm{Sj}$ & $\mathrm{Sh}^{+}$ & $\mathrm{Fb}, \mathrm{M}$ & GMH 5371 \\
\hline Solanaceae Juss. & & & & & & \\
\hline Capsicum annuиm $\mathrm{L}$. & Morich & Herb, er; cv & Af, Hs & All upazilas ${ }^{+}$ & $\mathrm{Sp}$ & MAR 3018 \\
\hline Cestrum diurnum $\mathrm{L}$. & Hasnahela & Shrub; pl & Hs & Ko, Sh, Ss & $\mathrm{M}, \mathrm{O}$ & MAR 3019 \\
\hline Datura metel L. & Sada dhutra & Shrub; w & $\mathrm{Fl}, \mathrm{Rs}$ & Sh, Ss, Ta & M & MAR 3323 \\
\hline Lycopersicon esculentum Mill. & Tomato & Herb, pr; cv & Af & All upazilas ${ }^{+}$ & $\mathrm{Vg}$ & KMNI 061 \\
\hline Nicotiana plumbaginifolia Viv. & Ban tamak & Herb, er; w & Af, Fl, Rs & As, Sh, Ss, Ta & $\mathrm{M}$ & $K M N I 028$ \\
\hline Petunia hybrida E. Vilm. & Petunia & Herb, er; pl & Hs, Rs & De, Ko, Ss & $\mathrm{O}$ & KMNI 064 \\
\hline
\end{tabular}


Table 1 contd.

\begin{tabular}{|c|c|c|c|c|c|c|}
\hline Scientific name & Bangla name & Habit & Habitat & Distribution & Use & RSE \\
\hline Physalis angulata $\mathrm{L}$. & Futka & Herb, er; w & $\mathrm{Fl}, \mathrm{Gl}, \mathrm{Rs}$ & $\mathrm{Ko}, \mathrm{Ss}$ & $\mathrm{M}$ & MAR 3020 \\
\hline$P$. minima $\mathrm{L}$. & Bon tepari & Herb, er; w & Af, Fl, Rs & All upazilas ${ }^{+}$ & M & KMNI 029 \\
\hline P. peruviana $\mathrm{L}$. & Tepari & Herb, er; w & Af, Fl, Rs & Ss & M & MAR 3021 \\
\hline Solanum americanum Mill. & Tit-begun & Herb, er; w & $\mathrm{Fl}, \mathrm{Gl}, \mathrm{Rs}$ & All upazilas & M & MAR 3022 \\
\hline S. indicum $\mathrm{L}$. & Rambegun & Shrub; w & Af, Fl, Rs & Ss, Ta & M & MAR 3023 \\
\hline S. melongena $\mathrm{L}$. & Begun & Shrub; w & Af, Hs & All upazilas ${ }^{+}$ & $\mathrm{Vg}$ & MAR 3024 \\
\hline S. sisymbriifolium Lam. & Katabegun & Herb, pr; w & $\mathrm{Fl}, \mathrm{Rs}$ & As, Sh, Ta & M & MAR 3025 \\
\hline S. torvum Sw. & Gota begun & Shrub; w & $\mathrm{Fl}, \mathrm{Sj}, \mathrm{Rs}$ & Ss, Ta & $\mathrm{M}, \mathrm{Vg}$ & MAR 3026 \\
\hline S. virginianum $\mathrm{L}$. & Kantikari & Herb, pr; w & $\mathrm{Sd}, \mathrm{Fm}$ & $\mathrm{Sh}^{+}$ & $\mathrm{M}, \mathrm{Vg}$ & MSR 3313 \\
\hline \multicolumn{7}{|l|}{ Convolvulaceae Juss. } \\
\hline $\begin{array}{l}\text { Aniseia martinicensis (Jacq.) } \\
\text { Choisy }\end{array}$ & Shadamati & Herb, cl; w & Ml, Rs & $\mathrm{Ka}, \mathrm{Sh}, \mathrm{Ss}$ & M & MAR 3027 \\
\hline $\begin{array}{l}\text { Argyreia roxburghii (Wall.) Arn. } \\
\text { ex Choisy }\end{array}$ & Argori lata & Herb, cl; w & $\mathrm{Sj}, \mathrm{Wl}$ & Ka & M & MAR 3028 \\
\hline $\begin{array}{l}\text { Camonea umbellata (L.) A.R. } \\
\text { Simões \& Staples }\end{array}$ & Goria lota & Herb, cl; w & $\mathrm{Fl}, \mathrm{Gl}, \mathrm{Rs}$ & Ko, Ss, Ta & $\mathrm{M}, \mathrm{O}$ & KMNI 032 \\
\hline Evolvulus nummularius (L.) L. & Bhui okra & Herb, cr; w & $\mathrm{Fl}, \mathrm{Gl}, \mathrm{Rs}$ & All upazilas ${ }^{+}$ & $\mathrm{M}, \mathrm{Sb}$ & KMNI 030 \\
\hline Ipomoea aquatica Forssk. & Kalmi shak & Herb, cr; w & Af, Wtl & All upazilas ${ }^{+}$ & $\mathrm{Vg}$ & KMNI 031 \\
\hline I. batatas (L.) Lam. & Misti alu & Herb, cr; cv & & De, Ko & $\mathrm{Vg}$ & MAR 3029 \\
\hline I. fistulosa Mart. ex Choisy & Dhol kolmi & Shrub; w & $\mathrm{Fl}, \mathrm{Ml}$ & All upazilas ${ }^{+}$ & $\mathrm{He}, \mathrm{Sb}$ & MAR 3030 \\
\hline I. littoralis Blume & Gang kolmi & Herb, cl; w & $\mathrm{Fm}, \mathrm{Sj}$ & $\mathrm{De}, \mathrm{Ka}$ & $\mathrm{M}, \mathrm{O}$ & MAR 3031 \\
\hline *I. pes-caprae (L.) R. Br. & Chagalkhuri & Herb, cr; w & Af, $\mathrm{Hs}$ & $\mathrm{Sh}^{+}$ & $\mathrm{M}, \mathrm{Sb}$ & GMH 5372 \\
\hline \multicolumn{7}{|l|}{ Cuscutaceae Dumort. } \\
\hline Cuscuta chinensis Lam. & Sharno lata & Herb, cl; w & Op & $\mathrm{De}, \mathrm{Ka}, \mathrm{Sh}$ & M & GMH 5373 \\
\hline C. reflexa Roxb. & Sharno lata & Herb, cl; w & Op & All upazilas ${ }^{+}$ & M & GMH 5374 \\
\hline \multicolumn{7}{|l|}{ Menyanthaceae Dumort. } \\
\hline $\begin{array}{l}\text { Nymphoides hydrophylla (Lour.) } \\
\text { Kuntze }\end{array}$ & Chand mala & Herb, fr; w & Wtl & As, Sh, Ss, Ta & M & KMNI 033 \\
\hline N. indica (L.) Kuntze & Panchuli mala & Herb, fr; w & Wtl & As, De, Sh, Ta & $\mathrm{M}, \mathrm{Vg}$ & KMNI 034 \\
\hline \multicolumn{7}{|l|}{ Hydroleaceae R. Br. ex Edwards } \\
\hline Hydrolea zeylanica (L.) Vahl & Kasschera & Herb, pr; w & Wtl & $\mathrm{Sh}, \mathrm{Ss}$ & M & MAR 3032 \\
\hline \multicolumn{7}{|l|}{ Boraginaceae Juss. } \\
\hline Heliotropium curassavicum L. & Nona-hatisur & Herb, pr; w & $\mathrm{Fl}, \mathrm{Rs}$ & $\mathrm{Sh}^{+}, \mathrm{Ss}$ & $\mathrm{M}, \mathrm{Vg}$ & GMH 5376 \\
\hline H. indicum $\mathrm{L}$. & Hatisur & Herb, er; w & Af, Fl, Rs & All upazilas ${ }^{+}$ & M & GMH 5377 \\
\hline Cordia dichotoma G. Forst. & Bohola, Bola & Tree, m; w & $\mathrm{Sj}, \mathrm{Wl}$ & Sh & Fw, M & GMH 5378 \\
\hline \multicolumn{7}{|l|}{ Verbenaceae J. St.-Hil. } \\
\hline Duranta erecta $\mathrm{L}$. & Duranto & Shrub; pl & Ml, Rs & $\mathrm{Ka}, \mathrm{Ko}, \mathrm{Sh}, \mathrm{Ss}$ & $\mathrm{He}, \mathrm{M}$ & MAR 3033 \\
\hline Lantana camara $\mathrm{L}$. & Kutus kanta & Shrub; w & $\mathrm{Rs}, \mathrm{Sj}, \mathrm{Wl}$ & $\mathrm{De}, \mathrm{Ka}, \mathrm{Ko}, \mathrm{Ta}$ & Fw, M & MAR 3034 \\
\hline $\begin{array}{l}\text { Lippia alba (Mill.) N.E. Br. ex } \\
\text { Britton \& P. Wilson }\end{array}$ & Motmotia & Shrub; w & $\mathrm{Fl}, \mathrm{Sj}$ & Ko, Sh, Ss, Ta & M & KMNI 035 \\
\hline Phyla nodiflora (L.) Greene & Vuiokra & Herb, cr; w & $\mathrm{Fl}, \mathrm{Gl}, \mathrm{Rs}$ & All upazilas ${ }^{+}$ & M & KMNI 036 \\
\hline \multicolumn{7}{|l|}{ Lamiaceae Martinov } \\
\hline Anisomeles indica (L.) Kuntze. & Gobura & Herb, er; w & $\mathrm{Fl}, \mathrm{Wl}$ & $\mathrm{De}, \mathrm{Ka}, \mathrm{Ko}, \mathrm{Ta}$ & M & GMH 5379 \\
\hline Clerodendrum indicum (L.) Kuntze & Bamunhatti & Shrub; w & $\mathrm{Fl}, \mathrm{Sj}, \mathrm{Wl}$ & $\mathrm{De}, \mathrm{Ka}, \mathrm{Ss}$ & M & GMH 5380 \\
\hline C. infortunatum $\mathrm{L}$. & Bhat & Shrub; w & $\mathrm{Fl}, \mathrm{Rs}, \mathrm{Wl}$ & All upazilas & M & GMH 5381 \\
\hline Gmelina arborea Roxb. & Gamari & Tree, $1 ; \mathrm{pl}$ & Rs, Wl & As, De, Ka, Sh & $\mathrm{M}, \mathrm{T}$ & GMH 5382 \\
\hline Hyptis capitata Jacq. & Tata tokma & Herb, er; w & $\mathrm{Fl}, \mathrm{Rs}, \mathrm{Sj}$ & As, Ko, Ss, Ta & M & GMH 5383 \\
\hline H. suaveolens (L.) Poit. & Tokma & Herb, er; w & $\mathrm{Fl}, \mathrm{Rs}, \mathrm{Sj}$ & Ko, Ss. Ta & M & GMH 5384 \\
\hline Leucas lavandulifolia $\mathrm{Sm}$. & Shetodron & Herb, er; w & Af, Fl, Rs & All upazilas & M & GMH 5385 \\
\hline
\end{tabular}


Table 1 contd.

\begin{tabular}{|c|c|c|c|c|c|c|}
\hline Scientific name & Bangla name & Habit & Habitat & Distribution & Use & RSE \\
\hline Leonurus sibiricus $\mathrm{L}$. & Rokto-dron & Herb, er; w & $\mathrm{Fl}, \mathrm{Rs}$ & $\mathrm{De}, \mathrm{Ko}, \mathrm{Ta}$ & $\mathrm{M}$ & GMH 5386 \\
\hline Ocimum americanum $\mathrm{L}$. & Bon tulashi & Herb, er; w & $\mathrm{Fl}, \mathrm{Hs}$ & $\mathrm{De}, \mathrm{Ka}, \mathrm{Ss}$ & M & GMH 5387 \\
\hline O. tenuiflorum $\mathrm{L}$. & Kalo tulsi & Herb, er; w & $\mathrm{Fl}, \mathrm{Hs}$ & Sh, Ta & M & GMH 5388 \\
\hline $\begin{array}{l}\text { Pogostemon benghalensis (Burm. } \\
\text { f.) Kuntze }\end{array}$ & Jui-lata & Shrub; w & Rs & $\mathrm{De}$ & M & GMH 5389 \\
\hline Premna serratifolia $\mathrm{L}$. & Gambari & Shrub; w & $\mathrm{Fm}, \mathrm{Sj}$ & $\mathrm{Ka}, \mathrm{Sh}^{+}$ & M & GMH 5390 \\
\hline $\begin{array}{l}\text { Rotheca serrata }(\text { L.) Steane \& } \\
\text { Mabb. }\end{array}$ & Bamanhati & Shrub; w & $\mathrm{Sj}, \mathrm{Wl}$ & $\mathrm{Ka}, \mathrm{Ta}$ & M & GMH 5391 \\
\hline Salvia splendens Sellow ex Schult. & Lal sagi & Herb, er; pl & Hs, Rs & Ss & $\mathrm{O}$ & GMH 5392 \\
\hline Tectona grandis $\mathrm{L} . \mathrm{f}$. & Shegun & Tree, $1 ; \mathrm{pl}$ & Rs, Wl & $\mathrm{De}, \mathrm{Ko}, \mathrm{Ss}$ & $\mathrm{T}$ & GMH 5393 \\
\hline Vitex negundo $\mathrm{L}$. & Nishinda & Shrub; w & $\mathrm{Fl}, \mathrm{Sj}, \mathrm{Rs}$ & $\mathrm{Sh}^{+}, \mathrm{Ss}, \mathrm{Ta}$ & M & GMH 5394 \\
\hline *Volkameria inermis L. & Shia vat & Shrub, li; w & $\mathrm{Fm}, \mathrm{Rb}$ & $\mathrm{Sh}^{+}$ & $\mathrm{M}, \mathrm{O}$ & GMH 5395 \\
\hline \multicolumn{7}{|l|}{ Plantaginaceae Juss. } \\
\hline Bacopa monnieri (L.) Wettst. & Brahmi & Herb, pr; w & $\mathrm{Fl}$, Wtl & $\mathrm{De}, \mathrm{Sh}^{+}$ & $\mathrm{M}, \mathrm{Vg}$ & MAR 3037 \\
\hline $\begin{array}{l}\text { Limnophila heterophylla (Roxb.) } \\
\text { Benth. }\end{array}$ & Patakutra & Herb, fr; w & Wtl & $\mathrm{Ka}, \mathrm{Sh}$ & Ap, M & MAR 3038 \\
\hline $\begin{array}{l}\text { Mecardonia procumbens (Mill.) } \\
\text { Small }\end{array}$ & Micardan & Herb, pr; w & $\mathrm{Fl}, \mathrm{Gl}, \mathrm{Rs}$ & All upazilas ${ }^{+}$ & M & MAR 3039 \\
\hline \multicolumn{7}{|l|}{ Oleaceae Hoffmanns. \& Link } \\
\hline Jasminum sambac (L.) Sol. & Beli & Shrub; pl & $\mathrm{Hs}$ & Ko, Ss & $\mathrm{M}, \mathrm{O}$ & MAR 3035 \\
\hline \multicolumn{7}{|l|}{$\begin{array}{l}\text { Linderniaceae Borsch, Kai Müll. } \\
\text { \& Eb. Fisch. }\end{array}$} \\
\hline Bonnaya antipoda (L.) Druce. & Zai ghas & Herb, pr; w & $\mathrm{Fl}, \mathrm{Gl}, \mathrm{Rs}$ & Ko, Sh, Ta & M & MAR 3042 \\
\hline Bonnaya ciliata (Colsm.) Spreng. & Bhui papri & Herb, pr; w & $\mathrm{Fl}, \mathrm{Gl}, \mathrm{Rs}$ & $\mathrm{De}, \mathrm{Ka}, \mathrm{Ss}$ & M & MAR 3043 \\
\hline $\begin{array}{l}\text { Lindernia anagallis (Burm. f.) } \\
\text { Pennell }\end{array}$ & Pani ghas & Herb, pr; w & $\mathrm{Fl}, \mathrm{Gl}, \mathrm{Rs}$ & Ko, Sh, Ss & M & MAR 3041 \\
\hline L. procumbens (Krock.) Borbás & Bakpuspa & Herb, pr; w & $\mathrm{Fl}, \mathrm{Gl}, \mathrm{Rs}$ & All upazilas ${ }^{+}$ & M & MAR 3045 \\
\hline L. rotundifolia (L.) Alston & Tan chapra & Herb, pr; w & $\mathrm{Fl}, \mathrm{Rs}, \mathrm{Wtl}$ & Ko, Sh & Ap, M & MAR 3046 \\
\hline $\begin{array}{l}\text { Torenia crustacea (L.) Cham. \& } \\
\text { Schltdl. }\end{array}$ & Chapra ghas & Herb, pr; w & $\mathrm{Fl}, \mathrm{Gl}, \mathrm{Rs}$ & Ko, Ta & M & MAR 3044 \\
\hline Torenia diffusa D. Don & Ushatoren & Herb, pr; w & $\mathrm{Fl}, \mathrm{Rs}$ & As, Sh, Ss, Ta & $\mathrm{O}$ & MAR 3047 \\
\hline \multicolumn{7}{|l|}{ Acanthaceae Juss. } \\
\hline *Acanthus ilicifolius $\mathrm{L}$. & Hargoza & Shrub; w & $\mathrm{Fm}, \mathrm{Rb}$ & $\mathrm{Sh}^{+}$ & M & GMH 5396 \\
\hline *A. volubilis Wall. & Lata hargoza & Herb, cl; w & $\mathrm{Fm}, \mathrm{Rb}$ & $\mathrm{Sh}^{+}$ & M & GMH 5397 \\
\hline $\begin{array}{l}\text { Andrographis paniculata (Burm. } \\
\text { f.) Nees }\end{array}$ & Kalomegh & Herb, er; w & $\mathrm{W} 1$ & $\mathrm{Ka}, \mathrm{Ss}$ & M & GMH 5398 \\
\hline *Avicennia marina (Forssk.) Vierh. & Morichabaen & Tree, m; w & $\mathrm{Wl}$ & $\mathrm{Sh}^{+}$ & $\mathrm{M}, \mathrm{T}$ & GMH 5399 \\
\hline *A. officinalis $\mathrm{L}$. & Baen & Tree, $1 ; \mathrm{w}$ & $\mathrm{Wl}$ & $\mathrm{Sh}^{+}$ & $\mathrm{M}, \mathrm{T}$ & GMH 5400 \\
\hline $\begin{array}{l}\text { Dipteracanthus prostratus (Poir.) } \\
\text { Nees }\end{array}$ & Posta booti & Herb, pr; w & $\mathrm{Fl}, \mathrm{Sj}, \mathrm{Wl}$ & As, De, Ka, Ko & M & GMH 5401 \\
\hline $\begin{array}{l}\text { Ecbolium ligustrinum (Vahl) } \\
\text { Vollesen }\end{array}$ & Shial leza & Herb, er; w & $\mathrm{Fl}, \mathrm{Wl}$ & $\mathrm{De}, \mathrm{Ka}, \mathrm{Ko}$ & M & GMH 5402 \\
\hline $\begin{array}{l}\text { Hemigraphis hirta (Vahl) T. } \\
\text { Anderson }\end{array}$ & Buripan & Herb, pr; w & $\mathrm{Fl}, \mathrm{Gl}, \mathrm{Rs}$ & All upazilas ${ }^{+}$ & M & GMH 5403 \\
\hline $\begin{array}{l}\text { Hygrophila auriculata } \\
\text { (Schumach.) Heine }\end{array}$ & Kulekhara & Herb, er; w & $\mathrm{Fl}$ & Ko & M & GMH 5404 \\
\hline H. difformis Blume & Bagua & Herb, fr; w & Wtl & $\mathrm{Ka}$, Ss & M & GMH 5405 \\
\hline H. erecta (Burm. f.) Hochr & Filareck & Herb, er; w & Wtl & $\mathrm{Sh}^{+}$ & M & GMH 5406 \\
\hline
\end{tabular}


Table 1 contd.

\begin{tabular}{|c|c|c|c|c|c|c|}
\hline Scientific name & Bangla name & Habit & Habitat & Distribution & Use & RSE \\
\hline H. phlomoides Nees & Gokul kanta & Herb, er; w & $\mathrm{Fl}$ & Ko, Ss, Ta & $\mathrm{M}$ & GMH 5407 \\
\hline H. polysperma (Roxb.) T. Anderson & Alai kalai & Herb, pr; w & $\mathrm{Fl}, \mathrm{Wtl}$ & $\mathrm{Sh}^{+}$ & M & MSR 3325 \\
\hline H. ringens var. ringens & Soza kulekhara & Herb, pr; w & $\mathrm{Fl}$, Wtl & $\mathrm{Sh}^{+}$ & M & MSR 3324 \\
\hline Justicia adhatoda $\mathrm{L}$. & Basok & Shrub; w & Ml, Rs & As, Ka, Sh, Ta & $\mathrm{He}, \mathrm{M}$ & GMH 5408 \\
\hline J. diffusa Willd. & Pitapapra & Herb, pr; w & $\mathrm{Fl}, \mathrm{Sj}$ & All upazilas & M & GMH 5409 \\
\hline J. gendarussa Burm. f. & Jagotmadan & Herb, er; w & $\mathrm{Fl}, \mathrm{Ml}, \mathrm{Sj}$ & $\mathrm{Sh}, \mathrm{Ss}$ & $\mathrm{He}, \mathrm{M}$ & GMH 5410 \\
\hline Nelsonia canescens (Lam.) Spreng. & Paramul & Herb, pr; w & $\mathrm{Fl}, \mathrm{Gl}, \mathrm{Wl}$ & All upazilas ${ }^{+}$ & M & GMH 5411 \\
\hline $\begin{array}{l}\text { Phaulopsis imbricata (Forssk.) } \\
\text { Sweet }\end{array}$ & Bhuiba shak & Herb, pr; w & $\mathrm{Sj}, \mathrm{Wl}$ & $\mathrm{Ka}, \mathrm{Ta}$ & M & GMH 5412 \\
\hline Ruellia tuberosa $\mathrm{L}$. & Chotpotey & Herb, er; w & $\mathrm{Fl}, \mathrm{Wl}$ & $\mathrm{Sh}, \mathrm{Ss}, \mathrm{Ta}$ & $\mathrm{M}, \mathrm{O}$ & GMH 5413 \\
\hline Rungia pectinata (L.) Nees & Pindi & Herb, pr; w & $\mathrm{Fl}, \mathrm{Gl}, \mathrm{Rs}$ & All upazilas ${ }^{+}$ & $\mathrm{P}$ & GMH 5414 \\
\hline $\begin{array}{l}\text { Thunbergia grandiflora (Roxb. ex } \\
\text { Rottl.) Roxb. }\end{array}$ & Neel lota & Herb, cl; w & Fm, Wl & As, Ka, Ko & M & GMH 5415 \\
\hline Pedaliaceae R. Br. & & & & & & \\
\hline $\begin{array}{l}\text { Sesamum indicum } \mathrm{L} \text {. } \\
\text { Bignoniaceae Juss. }\end{array}$ & Til & Herb, er; cv & Af & Ko & $\mathrm{M}, \mathrm{Oy}$ & KMNI 037 \\
\hline $\begin{array}{l}\text { Dolichandrone spathacea (L. f.) } \\
\text { Seem. }\end{array}$ & Gorshinga & Tree, $\mathrm{m} ; \mathrm{w}$ & Ml & Ss & $\mathrm{M}, \mathrm{T}$ & GMH 5416 \\
\hline Oroxylum indicum (L.) Kurz & Kanaidingi & Tree, m; w & $\mathrm{Sj}, \mathrm{Wl}$ & As, Ka, Ko & Dy, M & GMH 5417 \\
\hline $\begin{array}{l}\text { Tecoma stans (L.) Juss. ex Kunth } \\
\text { Lentibulariaceae Rich. }\end{array}$ & Tecoma & Tree, s; pl & Rs & Ss & $\mathrm{O}$ & GMH 5418 \\
\hline $\begin{array}{l}\text { Utricularia aurea Lour. } \\
\text { Phrymaceae Schauer }\end{array}$ & Patajhajhi & Herb, sm; w & Wtl & As, Sh, Ta & M & MAR 3048 \\
\hline $\begin{array}{l}\text { Mimulus orbicularis Wall. } \\
\text { Rubiaceae Juss. }\end{array}$ & Takabari & Herb, fr; w & Wtl & $\mathrm{Sh}$ & Ap, M & GMH 5419 \\
\hline $\begin{array}{l}\text { Coffea benghalensis B. Heyne ex } \\
\text { Schult. }\end{array}$ & Bangla coffee & Shrub; w & $\mathrm{Sj}$ & Ka, Ko & $\mathrm{M}, \mathrm{O}$ & $S A K 2136$ \\
\hline $\begin{array}{l}\text { Dentella repens (L.) J.R. Forst. \& } \\
\text { G. Forst. }\end{array}$ & Bhuipat & Herb, pr; w & Af, Fl, Gl & All upazilas & M & $S A K 2137$ \\
\hline Gardenia jasminoides J. Ellis & Gondhoraj & Shrub; pl & $\mathrm{Hs}$ & Ss & $\mathrm{M}, \mathrm{O}$ & $S A K 2138$ \\
\hline Hedytis diffusa Wild. & Panki & Herb, pr; w & $\mathrm{Fl}, \mathrm{Ml}, \mathrm{Rs}$ & All upazilas ${ }^{+}$ & M & GMH 5472 \\
\hline $\begin{array}{l}\text { Hypobathrum racemosum (Roxb.) } \\
\text { Kurz }\end{array}$ & Peetunga & Tree, s; w & Fm, Wl & $\mathrm{Ka}, \mathrm{Sh}^{+}$ & M & GMH 5470 \\
\hline Ixora coccinea $\mathrm{L}$. & Rangon & Shrub; pl & Hs, Rs & All upazilas & $\mathrm{O}$ & SAK 2139 \\
\hline I. cuneifolia Roxb. & Jangli rangon & Shrub; w & $\mathrm{Sj}, \mathrm{Wl}$ & $\mathrm{De}, \mathrm{Ka}$ & $\mathrm{M}, \mathrm{O}$ & $S A K 2140$ \\
\hline I. pavetta Andr. & Ban rangon & Shrub; w & $\mathrm{Sj}, \mathrm{Wl}$ & $\mathrm{Ka}, \mathrm{Sh}^{+}$ & $\mathrm{M}, \mathrm{O}$ & SAK 2141 \\
\hline Meyna spinosa Roxb. ex Link & Katai & Shrub; w & $\mathrm{Sj}, \mathrm{Wl}$ & $\mathrm{De}, \mathrm{Ka}, \mathrm{Ko}$ & M & $S A K 2142$ \\
\hline Morinda citrifolia $\mathrm{L}$. & Noni & Shrub; w & $\mathrm{Rb}, \mathrm{Wl}$ & $\mathrm{De}, \mathrm{Ka}$ & $\mathrm{M}$ & SAK 2143 \\
\hline $\begin{array}{l}\text { Mussaenda erythrophylla } \\
\text { Schumach. \& Thonn. }\end{array}$ & Lal mussenda & Shrub; pl & $\mathrm{Hs}$ & Ss & $\mathrm{O}$ & $S A K 2144$ \\
\hline M. philippica A. Rich. & Mussenda & Shrub; pl & Hs & Ko, Ss & $\mathrm{O}$ & $S A K 2145$ \\
\hline $\begin{array}{l}\text { Neolamarckia cadamba (Roxb.) } \\
\text { Bosser }\end{array}$ & Kadom & Tree, $1 ; \mathrm{w}$ & Rs, Wl & $\mathrm{De}, \mathrm{Ka}, \mathrm{Ss}$ & M, T & $S A K 2146$ \\
\hline Oldenlandia biflora $\mathrm{L}$. & Bhui papra & Herb, pr; w & $\mathrm{Fl}, \mathrm{Gl}$ & Ko, Ta & M & SAK 2147 \\
\hline O. corymbosa $\mathrm{L}$. & Khet papra & Herb, pr; w & Af, Fl, Gl & All upazilas ${ }^{+}$ & Dy, M & $S A K 2148$ \\
\hline O. diffusa (Willd.) Roxb. & Fussa papra & Herb, pr; w & Af, Fl, Gl & Ko, Sh, Ss & M & SAK 2149 \\
\hline O. verticillata $\mathrm{L}$. & Notapapra & Herb, pr; w & Af, Fl, Gl & De, Ss, Ta & M & $S A K 2150$ \\
\hline Pavetta indica $\mathrm{L}$. & Oriya, Jui & Shrub; w & $\mathrm{Sj}, \mathrm{Wl}$ & $\mathrm{De}, \mathrm{Ka}$ & $\mathrm{Co}, \mathrm{M}$ & SAK 2151 \\
\hline Spermacoce articularis L.f. & Baghajangla & Herb, pr; w & $\mathrm{Fl}, \mathrm{Ml}, \mathrm{Wl}$ & All upazilas & $\mathrm{M}$ & SAK 2152 \\
\hline
\end{tabular}


Table 1 contd.

\begin{tabular}{|c|c|c|c|c|c|c|}
\hline Scientific name & Bangla name & Habit & Habitat & Distribution & Use & RSE \\
\hline \multicolumn{7}{|l|}{ Asteraceae Bercht. \& J. Presl } \\
\hline Acmella calva (DC.) R.K. Jansen. & Surjakonnya & Herb, pr; w & $\mathrm{Fl}, \mathrm{Gl}, \mathrm{Rs}$ & All upazilas ${ }^{+}$ & M & SAK 2153 \\
\hline Ageratum conyzoides (L.) L. & Fulkuri & Herb, er; w & $\mathrm{Fl}, \mathrm{Ml}, \mathrm{Wl}$ & All upazilas & M & SAK 2154 \\
\hline Blumea lacera (Burm. f.) DC. & Shialmutra & Herb, er; w & $\mathrm{Fl}, \mathrm{Gl}, \mathrm{Rs}$ & All upazilas ${ }^{+}$ & M & SAK 2155 \\
\hline Blumea membranacea Wall. ex DC. & Kukurshinga & Herb, er; w & $\mathrm{Fl}, \mathrm{Gl}, \mathrm{Rs}$ & Sh, Ta & M & SAK 2156 \\
\hline $\begin{array}{l}\text { Chromolaena odorata (L.) R.M. } \\
\text { King \& H. Rob. }\end{array}$ & Assam-lata & Herb, er; w & $\mathrm{Fl}, \mathrm{Fm}, \mathrm{Wl}$ & $\mathrm{De}, \mathrm{Ko}, \mathrm{Ss}, \mathrm{Ta}$ & M & $S A K 2157$ \\
\hline $\begin{array}{l}\text { Conyza semipinnatifida Wall. ex } \\
\text { DC. }\end{array}$ & Coniza & Herb, er; w & $\mathrm{Fl}, \mathrm{Rs}$ & $\mathrm{Sh}^{+}$ & M & $S A K 2158$ \\
\hline Cosmos bipinnatus Cav. & Cosmos & Herb, er; pl & Hs, Rs & $\mathrm{De}, \mathrm{Ko}, \mathrm{Ss}$ & $\mathrm{O}$ & KMNI 065 \\
\hline Cyanthillium cinereum (L.) H. Rob. & Kukshim & Herb, er; w & $\mathrm{Fl}, \mathrm{Gl}, \mathrm{Rs}$ & All upazilas ${ }^{+}$ & M & SAK 2159 \\
\hline Dahlia imperialis Roezl ex Ortgies & Dalia & Herb, er; pl & Hs, Rs & $\mathrm{Ko}, \mathrm{Ss}$ & $\mathrm{O}$ & KMNI 066 \\
\hline Eclipta prostrata (L.) L. & Kalokeshi & Herb, pr; w & $\mathrm{Fl}, \mathrm{Gl}, \mathrm{Rs}$ & All upazilas ${ }^{+}$ & Dy, M & SAK 2160 \\
\hline Emilia sonchifolia $(\mathrm{L}$.$) DC.$ & Mechitra & Herb, er; w & $\mathrm{Fl}, \mathrm{Gl}, \mathrm{Rs}$ & $\mathrm{De}, \mathrm{Ko}, \mathrm{Ss}, \mathrm{Ta}$ & $M$ & SAK 2161 \\
\hline Enydra fluctuans DC. & Helencha & Herb, pr; w & Wtl & $\mathrm{De}, \mathrm{Sh}, \mathrm{Ss}, \mathrm{Ta}$ & $\mathrm{M}, \mathrm{Vg}$ & SAK 2162 \\
\hline $\begin{array}{l}\text { Glebionis coronaria (L.) Cass. ex } \\
\text { Spach }\end{array}$ & Chandramallika & Herb, er; pl & Hs, Rs & $\mathrm{Ko}, \mathrm{Ss}$ & $\mathrm{O}$ & GMH 5467 \\
\hline Gnaphalium polycaulon Pers. & Bara kamra & Herb, er; w & $\mathrm{Fl}, \mathrm{Gl}$ & $\mathrm{De}, \mathrm{Ko}, \mathrm{Ss}$ & M & SAK 2163 \\
\hline Grangea maderaspatana (L.) Poir. & Namuti & Herb, er; w & Af, Fl, Ml & Ko, Ss, Ta & M & SAK 2164 \\
\hline Helianthus aпnииs L. & Surjomukhi & Herb, er; pl & Af, Hs & As, De, Ss, Ta & $\mathrm{O}, \mathrm{Oy}$ & GMH 5468 \\
\hline Launaea asplenifolia Hook. f. & Tik-chana & Herb, er; w & $\mathrm{Fl}, \mathrm{Rs}$ & $\mathrm{Sh}^{+}$ & $\mathrm{M}$ & SAK 2165 \\
\hline $\begin{array}{l}\text { Mikania cordata (Burm. f.) B.L. } \\
\text { Rob. }\end{array}$ & Assam-lata & Herb, cl; w & $\mathrm{Sj}, \mathrm{Fm}, \mathrm{Wl}$ & All upazilas ${ }^{+}$ & M & SAK 2166 \\
\hline Parthenium hysterophorus L. & Parthenum & Herb, er; w & $\mathrm{Fl}, \mathrm{Rs}$ & $\mathrm{De}, \mathrm{Sh}, \mathrm{Ss}, \mathrm{Ta}$ & M & SAK 2167 \\
\hline $\begin{array}{l}\text { Pseudognaphalium luteoalbum (L.) } \\
\text { Hilliard \& B.L. Burtt }\end{array}$ & Barakamra & Herb, er; w & $\mathrm{Fl}, \mathrm{Gl}, \mathrm{Rs}$ & Ko, Sh, Ss & M & SAK 2168 \\
\hline Sonchus arvensis $\mathrm{L}$. & Chashar & Herb, er; w & $\mathrm{Fl}, \mathrm{Gl}, \mathrm{Rs}$ & $\mathrm{Ta}$ & M & SAK 2169 \\
\hline Sphaeranthus indicus $\mathrm{L}$. & Mundi & Herb, pr; w & Af, Fl, Rs & Ko, Ss & M & SAK 2170 \\
\hline Sphagneticola trilobata (L.) Pruski & Bhringaraj & Herb, pr; w & $\mathrm{Fl}, \mathrm{Rs}$ & Ss & $\mathrm{Gm}, \mathrm{O}$ & SAK 2171 \\
\hline Synedrella nodiflora (L.) Gaertn. & Nakphul & Herb, er; w & $\mathrm{Fl}, \mathrm{Gl}, \mathrm{Rs}$ & $\mathrm{Ka}, \mathrm{Ko}, \mathrm{Ss}, \mathrm{Ta}$ & M & $S A K 2172$ \\
\hline Tagetes erecta $\mathrm{L}$. & Gada & Herb, er; pl & Hs, Rs & All upazilas ${ }^{+}$ & $\mathrm{M}, \mathrm{O}$ & GMH 5469 \\
\hline Tridax procumbens (L.) L. & Tridhara & Herb, er; w & $\mathrm{Fl}, \mathrm{Gl}, \mathrm{Rs}$ & All upazilas & $\mathrm{M}$ & $S A K 2173$ \\
\hline \multicolumn{7}{|l|}{ LILIOPSIDA Batsch } \\
\hline $\begin{array}{l}\text { Alismataceae Vent. } \\
\text { Sagittaria sagittifolia L. } \\
\text { Hydrocharitaceae Juss. }\end{array}$ & Chotokut & Herb, er; w & Af, Wtl & Sh, Ta & $\mathrm{O}, \mathrm{Lf}$ & MAR 3070 \\
\hline Hydrilla verticillata (L. f.) Royle & Kureli & Herb, sm; w & Wtl & $\mathrm{De}, \mathrm{Sh}, \mathrm{Ss}$ & Ap, $M$ & GMH 5421 \\
\hline Najas indica (Willd.) Cham. & Deshijhaji & Herb, sm; w & Wtl & $\mathrm{De}, \mathrm{Sh}, \mathrm{Ss}$ & Ap, Gm & GMH 5422 \\
\hline N. minor All. & Borojhaji & Herb, sm; w & Wtl & As, De, Sh, Ss & $\mathrm{Ff}, \mathrm{Gm}$ & GMH 5423 \\
\hline Ottelia alismoides (L.) Pers. & Panikala & Herb, sm; w & Wtl & As, $\mathrm{Ka}, \mathrm{Ss}$ & $\mathrm{M}, \mathrm{Vg}$ & GMH 5424 \\
\hline $\begin{array}{l}\text { Vallisneria spiralis } \mathrm{L} . \\
\text { Aponogetonaceae Planch. }\end{array}$ & Patajhangi & Herb, sm; w & Wtl & $\mathrm{Sh}, \mathrm{Ta}$ & Ap, $M$ & GMH 5425 \\
\hline $\begin{array}{l}\text { Aponogeton appendiculatus } \mathrm{H} \text {. } \\
\text { Bruggen }\end{array}$ & Ghechu & Herb, sm; w & Wtl & Sh, Ss & Ap, $M$ & MAR 3115 \\
\hline \multicolumn{7}{|l|}{$\begin{array}{l}\text { Potamogetonaceae Bercht. \& J. } \\
\text { Presl }\end{array}$} \\
\hline Potamogeton crispus $\mathrm{L}$. & Pata zhanchi & Herb, sm; w & Wtl & $\mathrm{Sh}, \mathrm{Ss}, \mathrm{Ta}$ & $\mathrm{M}, \mathrm{Wp}$ & MAR 3049 \\
\hline P. nodosus Poir. & Lombu zhanchi & Herb, fr; w & Wtl & As, Sh, Ss & $\mathrm{M}, \mathrm{Wp}$ & MAR 3050 \\
\hline
\end{tabular}


Table 1 contd.

\begin{tabular}{|c|c|c|c|c|c|c|}
\hline Scientific name & Bangla name & Habit & Habitat & Distribution & Use & RSE \\
\hline \multicolumn{7}{|l|}{ Arecaceae Bercht. \& J. Presl } \\
\hline Areca catechu $\mathrm{L}$. & Supari & Palm; pl & Hs, Rs & All upazilas & Dy, M & MAR 3051 \\
\hline Borassus flabellifer $\mathrm{L}$. & Tal & Palm; pl & Hs, Rs & All upazilas & $\mathrm{Fb}, \mathrm{M}$ & MAR 3052 \\
\hline $\begin{array}{l}\text { Calamus guruba Buch.-Ham. ex } \\
\text { Mart. }\end{array}$ & Jalibet & Palm; pl & $\mathrm{Ml}, \mathrm{Rb}, \mathrm{Sj}$ & $\mathrm{De}, \mathrm{Ka}$ & $\mathrm{Hc}$ & MAR 3053 \\
\hline C. longisetus Griff. & Karakbet & Palm; pl & Ml & $\mathrm{De}, \mathrm{Ka}, \mathrm{Ko}$ & $\mathrm{Hc}$ & MAR 3054 \\
\hline C. tenuis Roxb. & Jalibet & Palm; pl & Ml & $\mathrm{Ka}, \mathrm{Ss}$ & $\mathrm{Hc}, \mathrm{M}$ & MAR 3055 \\
\hline Caryota urens $\mathrm{L}$. & Fishtail palm & Palm; pl & Ml, Rs & Ss & $\mathrm{M}, \mathrm{O}$ & MAR 3056 \\
\hline Chamaedorea elegans Mart. & Areca palm & Palm; pl & Hs, Ml, Rs & Ss & $\mathrm{Fb}, \mathrm{O}$ & MAR 3057 \\
\hline Cocos nucifera $\mathrm{L}$. & Narikel & Palm; pl & Hs, Ml, Rs & All upazilas ${ }^{+}$ & $\mathrm{Fb}, \mathrm{Fr}$ & MAR 3058 \\
\hline Elaeis guineensis Jacq. & Oil palm & Palm; pl & Hs , Ml & $\mathrm{De}, \mathrm{Ka}, \mathrm{Ss}$ & $\mathrm{M}, \mathrm{Oy}$ & MAR 3059 \\
\hline $\begin{array}{l}\text { Livistona chinensis (Jacq.) R. Br. } \\
\text { ex Mart. }\end{array}$ & China tokopata & Palm; pl & Ml, Rs & Ss & $\mathrm{Fb}, \mathrm{Hc}$ & MAR 3060 \\
\hline *Nypa fruticans Wurmb & Golpatta & Palm; w & $\mathrm{Rb}, \mathrm{Fm}$ & $\mathrm{Sh}^{+}$ & $\mathrm{M}, \mathrm{Tm}$ & GMH 5426 \\
\hline *Phoenix paludosa Roxb. & Hental & Palm; w & Fm, Wl & $\mathrm{Sh}^{+}$ & $\mathrm{Fr}, \mathrm{Hc}$ & GMH 5427 \\
\hline P. sylvestris (L.) Roxb. & Deshi khejur & Palm; w & Ml, Rs & All upazilas ${ }^{+}$ & $\mathrm{Ju}, \mathrm{M}$ & MAR 3061 \\
\hline Rhapis excelsa (Thunb.) Henry & Gurital & Palm; pl & Hs & Ss & $\mathrm{O}$ & MAR 3062 \\
\hline $\begin{array}{l}\text { Roystonea regia (Kunth) O.F. } \\
\text { Cook }\end{array}$ & Bottol palm & Palm; pl & Ml, Rs & Ss & $\mathrm{O}, \mathrm{Tm}$ & MAR 3063 \\
\hline \multicolumn{7}{|l|}{ Pandanaceae R. Br. } \\
\hline Pandanus amaryllifolius Roxb. & Polau pata & Herb, er; pl & $\mathrm{Fl}, \mathrm{Hs}$ & Ss & M, Pf & MAR 3064 \\
\hline P. foetidus Roxb. & Keya kanta & Shrub; w & $\mathrm{Fl}, \mathrm{Rb}$ & $\mathrm{De}, \mathrm{Ka}$ & $\mathrm{M}, \mathrm{O}$ & MAR 3065 \\
\hline \multicolumn{7}{|l|}{ Araceae Juss. } \\
\hline Alocasia fornicata (Roxb.) Schott & Bish kachu & Herb, er; w & $\mathrm{Fl}, \mathrm{Wl}$ & $\mathrm{De}, \mathrm{Ka}, \mathrm{Ko}, \mathrm{Ta}$ & $\mathrm{M}$ & MAR 3066 \\
\hline A. macrorrhizos (L.) G. Don & Man kachu & Herb, er; cv & $\mathrm{Fl}, \mathrm{Hs}$ & All upazilas & $\mathrm{Vg}$ & GMH 5428 \\
\hline $\begin{array}{l}\text { Amorphophallus paeoniifolius } \\
\text { (Dennst.) Nicolson }\end{array}$ & Ol kachu & Herb, er; cv & Af, $\mathrm{Hs}$ & All upazilas & $\mathrm{Vg}$ & GMH 5429 \\
\hline Caladium bicolor (Aiton) Vent. & Diranga kachu & Herb, er; pl & Hs & $\mathrm{De}, \mathrm{Ka}, \mathrm{Ss}$ & $\mathrm{O}$ & MAR 3067 \\
\hline Colocasia esculenta (L.) Schott & Kachu & Herb, er; w & Af, Fl, Wtl & All upazilas ${ }^{+}$ & $\mathrm{Vg}$ & KMNI 039 \\
\hline $\begin{array}{l}\text { Cryptocoryne ciliata (Roxb.) Fisch. } \\
\text { ex Wydler }\end{array}$ & Kerali & Herb, er; w & $\mathrm{Fl}, \mathrm{Rb}, \mathrm{Wtl}$ & $\mathrm{De}, \mathrm{Sh}^{+}$ & $\mathrm{M}, \mathrm{Sb}$ & GMH 5430 \\
\hline $\begin{array}{l}\text { Epipremnum aureum (Linden \& } \\
\text { André) G.S. Bunting }\end{array}$ & Money plant & Herb, cl; w & Hs, Wl & Ss & $\mathrm{O}$ & MAR 3068 \\
\hline Lasia spinosa (L.) Thwaites & Kata kachu & Herb, er; w & $\mathrm{Fl}, \mathrm{Hs}$, Wtl & $\mathrm{De}, \mathrm{Ka}, \mathrm{Ko}$ & $\mathrm{M}, \mathrm{Vg}$ & GMH 5431 \\
\hline Lemna minor $\mathrm{L}$. & Sujipana & Herb, ff; w & Wtl & Ss, Ta & $\mathrm{Ff}, \mathrm{Wp}$ & KMNI 040 \\
\hline L. perpusilla Torr. & Khudipana & Herb, ff; w & Wtl & All upazilas ${ }^{+}$ & Ff, Wp & KMNI 041 \\
\hline Pistia stratiotes L. & Topapana & Herb, ff; w & Wtl & Ko, Ss, Ta & $\mathrm{M}$ & GMH5 432 \\
\hline Syngonium podophyllum Schott & Podolata kachu & Herb, pr; w & $\mathrm{Fl}, \mathrm{Hs}, \mathrm{Sj}$ & $\mathrm{Ka}$, Ss & $\mathrm{O}$ & MAR 3069 \\
\hline $\begin{array}{l}\text { Typhonium flagelliforme (Lodd.) } \\
\text { Blume }\end{array}$ & Ghechu & Herb, er; w & Af, $\mathrm{Fl}, \mathrm{Rs}$ & $\mathrm{De}, \mathrm{Ko}, \mathrm{Ss}$, Ta & M & GMH 5433 \\
\hline T. trilobatum (L.) Schott & Ghet kachu & Herb, er; w & $\mathrm{Fl}, \mathrm{Rs}$ & All upazilas & $\mathrm{M}, \mathrm{Vg}$ & GMH 5434 \\
\hline $\begin{array}{l}\text { Xanthosoma sagittifolium (L.) } \\
\text { Schott }\end{array}$ & Dudh kachu & Herb, er; w & $\mathrm{Ml}, \mathrm{Hs}$ & As, Ka, Ko, Sh & $\mathrm{M}, \mathrm{Vg}$ & GMH 5435 \\
\hline Commelinaceae Mirb. & & & & & & \\
\hline Commelina benghalensis $\mathrm{L}$. & Kanshira & Herb, cr; w & Af, Fl, Rs & All upazilas ${ }^{+}$ & Dy, M & MAR 3071 \\
\hline C. diffusa Burm. f. & Kanshira & Herb, cr; w & Af, Fl, Rs & $\mathrm{De}, \mathrm{Ko}, \mathrm{Ss}, \mathrm{Ta}$ & Dy, M & KMNI 042 \\
\hline C. erecta $\mathrm{L}$. & Jata kanchira & Herb, er; w & $\mathrm{Fl}, \mathrm{Gl}, \mathrm{Rs}$ & As, Ko, Ss & M & KMNI 043 \\
\hline C. longifolia $\mathrm{Lam}$. & Pani kanshira & Herb, cr; w & $\mathrm{Fl}, \mathrm{Gl}, \mathrm{Rs}$ & $\mathrm{Ko}, \mathrm{Sh}^{+}, \mathrm{Ta}$ & M & KMNI 044 \\
\hline $\begin{array}{l}\text { Cyanotis axillaris (L.) D. Don ex } \\
\text { Sweet }\end{array}$ & Baghanulla & Herb, pr; w & Af, Fl, Gl & $\mathrm{De}, \mathrm{Ka}, \mathrm{Ko}, \mathrm{Ss}$ & M & MAR 3072 \\
\hline C. cristata (L.) D. Don. & Kanai & Herb, cr; w & Af, Fl, Gl & $\mathrm{De}, \mathrm{Ko}, \mathrm{Ta}$ & M & MAR 3073 \\
\hline Murdannia nudiflora (L.) Brenan & Kureli & Herb, cr; w & $\mathrm{Fl}, \mathrm{Ml}$ & All upazilas & $\mathrm{M}$ & MAR 3074 \\
\hline
\end{tabular}


Table 1 contd.

\begin{tabular}{|c|c|c|c|c|c|c|}
\hline Scientific name & Bangla name & Habit & Habitat & Distribution & Use & RSE \\
\hline \multicolumn{7}{|l|}{ Cyperaceae Juss. } \\
\hline $\begin{array}{l}\text { Bulbostylis barbata (Rottb.) C.B. } \\
\text { Clarke }\end{array}$ & Bulbobata & Herb, er; w & Gl & $\mathrm{Sh}, \mathrm{Ss}$ & $\mathrm{Lf}, \mathrm{Sb}$ & MAR 3075 \\
\hline Cyperus compressus $\mathrm{L}$. & Chancha & Herb, er; w & Af, Fl, Gl & All upazilas & M & MAR 3076 \\
\hline C. cuspidatus Kunth & Sagarmuthi & Herb, er; w & $\mathrm{Fl}, \mathrm{Gl}, \mathrm{Rs}$ & Sh, Ss, Ta & M & MAR 3077 \\
\hline C. difformis $\mathrm{L}$. & Behua ghasi & Herb, er; w & Af, Fl & Ko, Ss, Ta & M & MAR 3078 \\
\hline C. eragrostis Lam. & Bada ghas & Herb, er; w & $\mathrm{Fl}, \mathrm{Gl}, \mathrm{Rs}$ & Ss, Ta & $\mathrm{Lf}, \mathrm{Sb}$ & MAR 3079 \\
\hline C. exaltatus Retz. & Tata ghasi & Herb, er; w & $\mathrm{Gl}, \mathrm{Wtl}$ & $\mathrm{Ta}$ & $\mathrm{M}, \mathrm{Tm}$ & GMH 5436 \\
\hline C. iria $\mathrm{L}$. & Barachucha & Herb, er; w & $\mathrm{Fl}, \mathrm{Gl}, \mathrm{Rs}$ & $\mathrm{Ka}, \mathrm{Sh}^{+}, \mathrm{Ss}, \mathrm{Ta}$ & $\mathrm{M}, \mathrm{Lf}$ & GMH 5437 \\
\hline C. malaccensis Lam. & Shumati pati & Herb, er; w & Wtl & $\mathrm{Sh}+, \mathrm{Ss}$ & $\mathrm{Hc}, \mathrm{M}$ & GMH 5438 \\
\hline C. pangorei Rottb. & Madurkathi & Herb, er; w & $\mathrm{Fl}, \mathrm{Wtl}$ & Ss, Ta & $\mathrm{Hc}, \mathrm{Lf}$ & MAR 3080 \\
\hline C. rotundus $\mathrm{L}$. & Nagarmutha & Herb, er; w & $\mathrm{Fl}, \mathrm{Gl}, \mathrm{Rs}$ & All upazilas & $\mathrm{Hc}, \mathrm{M}$ & KMNI 045 \\
\hline C. tenuiculmis Boeckeler & Tonimutha & Herb, er; w & Wtl & $\mathrm{Sh}^{+}$ & $\mathrm{Lf}$ & MSR 3326 \\
\hline $\begin{array}{l}\text { Eleocharis dulcis (Burm. f.) Trin. } \\
\text { ex Hensch. }\end{array}$ & Mishti ghasi & Herb, er; w & $\mathrm{Fl}, \mathrm{Ml}$ & $\mathrm{Sh}^{+}$ & $\mathrm{M}, \mathrm{Vg}$ & MSR 3331 \\
\hline Fimbristylis dichotoma (L.) Vahl & Bara nirbishi & Herb, er; w & $\mathrm{Ml}, \mathrm{Wtl}$ & $\mathrm{Ko}, \mathrm{Sh}^{+}, \mathrm{Ss}$ & $\mathrm{Gm}, \mathrm{Sb}$ & GMH 5439 \\
\hline F. ferruginea $(\mathrm{L}$.$) Vahl$ & Gini fimbry & Herb, er; w & $\mathrm{Gl}, \mathrm{Wtl}$ & $\mathrm{Sh}^{+}$ & $\mathrm{Sb}, \mathrm{Tm}$ & MSR 3335 \\
\hline Fuirena ciliaris (L.) Roxb. & Poshmi ghas & Herb, er; w & $\mathrm{Fl}, \mathrm{Gl}, \mathrm{Wtl}$ & Ko, Ss, Sh, Ta & $\mathrm{Lf}$ & MAR 3080 \\
\hline Kyllinga brevifolia Rottb. & Shabujnirbisa & Herb, er; w & Af, Fl, Gl & All upazilas & Lf, M & KMNI 046 \\
\hline K. bulbosa P. Beauv. & Golanirbisa & Herb, er; w & Af, Fl, Gl & As, Ka, Ss, Ta & $\mathrm{Lf}, \mathrm{M}$ & MAR 3081 \\
\hline $\begin{array}{l}\text { K. nemoralis (J.R. Forst. \& G. } \\
\text { Forst.) Dandy ex Hutch. \& Dalziel }\end{array}$ & Subashinirbisa & Herb, er; w & Af, Fl, Gl & All upazilas & Lf, M & KMNI 047 \\
\hline $\begin{array}{l}\text { Pycreus polystachyos (Rottb.) P. } \\
\text { Beauv. }\end{array}$ & Pikppli ghas & Herb, er; w & $\mathrm{Gl}, \mathrm{Wtl}$ & $\mathrm{Sh}^{+}$ & $\mathrm{Lf}, \mathrm{Sb}$ & MSR 3340 \\
\hline P. uniloides (R. Br.) Urb. & Paikol ghas & Herb, er; w & $\mathrm{Fl}, \mathrm{Gl}, \mathrm{Ml}$ & $\mathrm{Sh}^{+}$ & $\mathrm{Lf}, \mathrm{Sb}$ & MSR 3332 \\
\hline $\begin{array}{l}\text { Schoenoplectiella articulata (L.) } \\
\text { Lye }\end{array}$ & Chechra & Herb, er; w & Af, Fl, Wtl & Ko, Ss, Sh, Ta & Lf, M & GMH 5440 \\
\hline \multicolumn{7}{|l|}{ Poaceae Barnhart } \\
\hline $\begin{array}{l}\text { Axonopus compressus (Sw.) P. } \\
\text { Beauv. }\end{array}$ & Karpet ghas & Herb, er; w & $\mathrm{Fl}, \mathrm{Gl}, \mathrm{Rs}$ & All upazilas ${ }^{+}$ & $\mathrm{Lf}, \mathrm{Sb}$ & KMNI 048 \\
\hline Bambusa balcooa Roxb. & Borak bans & Bamboo; w & $\mathrm{Wl}$ & $\mathrm{De}, \mathrm{Ka}, \mathrm{Ko}, \mathrm{Ss}$ & $\mathrm{Hc}, \mathrm{Vg}$ & MAR 3082 \\
\hline B. nutans Wall. ex Munro & Mahal bans & Bamboo; w & $\mathrm{Wl}$ & As, Ko, Ss, Ta & $\mathrm{Pp}, \mathrm{Tm}$ & MAR 3083 \\
\hline B. tulda Roxb. & Mirtinga & Bamboo; w & Hs, Wl & $\mathrm{Ka}, \mathrm{Ko}, \mathrm{Ss}$ & $\mathrm{Hc}, \mathrm{Pp}$ & MAR 3084 \\
\hline Brachiaria distachya (L.) Stapf & Cori ghas & Herb, cr; w & Gl, Rs & All upazilas & $\mathrm{Lf}, \mathrm{Sb}$ & GMH 5441 \\
\hline $\begin{array}{l}\text { Chrysopogon aciculatus (Retz.) } \\
\text { Trin. }\end{array}$ & Prem kanta & Herb, er; w & Gl, Rs & All upazilas & $\mathrm{Hc}, \mathrm{Sb}$ & GMH 5442 \\
\hline C. zizanioides (L.) Roberty & Bena & Herb, er; w & $\mathrm{Fl}, \mathrm{Ml}$ & $\mathrm{Ka}, \mathrm{Ss}$, Ta & $\mathrm{M}, \mathrm{Sb}$ & KMNI 049 \\
\hline Coix lacryma-jobi $\mathrm{L}$. & Tasbi & Herb, er; w & $\mathrm{Fl}, \mathrm{Wtl}$ & Ss & $\mathrm{Hc}, \mathrm{M}$ & KMNI 050 \\
\hline Cynodon dactylon (L.) Pers. & Durba ghas & Herb, pr; w & Af, Fl, Gl & All upazilas ${ }^{+}$ & $\mathrm{M}, \mathrm{Sb}$ & KMNI 051 \\
\hline Cyrtococcum patens (L.) A. Camus & Cyrtococcum & Herb, pr; w & Gl, Rs, Wl & $\mathrm{De}, \mathrm{Ka}$ & Lf & MAR 3085 \\
\hline $\begin{array}{l}\text { Dactyloctenium aegyptium (L.) } \\
\text { Willd. }\end{array}$ & Kakpaya & Herb, er; w & $\mathrm{Fl}, \mathrm{Gl}, \mathrm{Rs}$ & As, Sh, Ss, Ta & $\mathrm{Lf}, \mathrm{Sb}$ & GMH 5443 \\
\hline Digitaria ciliaris (Retz.) Koeler & Kokjachira & Herb, er; w & $\mathrm{Fl}, \mathrm{Gl}, \mathrm{Rs}$ & Ss, Ta & $\mathrm{Gm}, \mathrm{Sb}$ & MAR 3086 \\
\hline D. longiflora (Retz.) Pers. & Kanka-juriya & Herb, pr; w & Af, Gl, Rs & Ss & $\mathrm{Lf}, \mathrm{Sb}$ & MAR 3087 \\
\hline Echinochloa colona (L.) Link. & Shama ghas & Herb, er; w & Af, Fl, Gl & All upazilas & $\mathrm{Lf}, \mathrm{Sb}$ & MAR 3088 \\
\hline E. crus-galli (L.) P. Beauv. & Barashama ghas & Herb, er; w & $\mathrm{Af}, \mathrm{Fl}, \mathrm{Gl}$ & $\mathrm{Sh}^{+}, \mathrm{Ss}, \mathrm{Ta}$ & Lf, M & MAR 3089 \\
\hline Eleusine indica (L.) Gaertn. & Malankuri & Herb, er; w & $\mathrm{Af}, \mathrm{Fl}, \mathrm{Gl}$ & All upazilas & $\mathrm{M}, \mathrm{Sb}$ & KMNI 052 \\
\hline $\begin{array}{l}\text { Eragrostis amabilis (L.) Wight \& } \\
\text { Arn. }\end{array}$ & Koni ghas & Herb, er; w & $\mathrm{Af}, \mathrm{Fl}, \mathrm{Gl}$ & $\mathrm{De}, \mathrm{Ka}, \mathrm{Ss}$ & $\mathrm{O}, \mathrm{Sb}$ & MAR 3090 \\
\hline E. tremula Hochst. ex Steud. & Chiranula & Herb, er; w & $\mathrm{Fl}, \mathrm{Gl}, \mathrm{Rs}$ & As, Ka, Ss, Ta & $\mathrm{Lf}, \mathrm{Tm}$ & MAR 3091 \\
\hline Hemarthria protensa Steud. & Chaila & Herb, er; w & Ml, Wtl & Ss & $\mathrm{Lf}, \mathrm{Sb}$ & MAR 3092 \\
\hline
\end{tabular}


Table 1 contd.

\begin{tabular}{|c|c|c|c|c|c|c|}
\hline Scientific name & Bangla name & Habit & Habitat & Distribution & Use & RSE \\
\hline $\begin{array}{l}\text { Hygroryza aristata (Retz.) Nees ex } \\
\text { Wight \& Arn. }\end{array}$ & Jongli dhan & Herb, er; w & $\mathrm{Wtl}$ & As, Ta & Lf, $M$ & GMH 5444 \\
\hline Isachne globosa (Thunb.) Kuntze & Isacdana & Herb, er; w & $\mathrm{Gl}, \mathrm{Fl}$ & Ko, Ss & $\mathrm{Lf}, \mathrm{Sb}$ & MAR 3093 \\
\hline Imperata cylindrica (L.) Raeusch. & Chhan & Herb, er; w & Gr, Ml, Rs & All upazilas & $\mathrm{Sb}, \mathrm{Tm}$ & GMH 5445 \\
\hline Leersia hexandra Sw. & Arali & Herb, pr; w & Wtl & As, Ss, Ta & Lf & GMH 5446 \\
\hline Leptochloa chinensis (L.) Nees & Phulka ghas & Herb, er; w & Af & Ss, Ta & $\mathrm{Lf}$ & MAR 3094 \\
\hline $\begin{array}{l}\text { *Myriostachya wightiana (Nees ex } \\
\text { Steud.) Hook. f. }\end{array}$ & Balia ghas & Herb, er; w & $\mathrm{Fm}, \mathrm{Wl}$ & $\mathrm{Sh}^{+}$ & $\mathrm{Lf}, \mathrm{Tm}$ & GMH 5447 \\
\hline $\begin{array}{l}\text { Oplismenus burmanni (Retz.) P. } \\
\text { Beauv. }\end{array}$ & Jabri durba & Herb, er; w & $\mathrm{Fl}, \mathrm{Rs}, \mathrm{Wl}$ & All upazilas & Lf & GMH 5448 \\
\hline O. compositus (L.) P. Beauv. & Gohur & Herb, er; w & $\mathrm{Fl}, \mathrm{Rs}, \mathrm{Wl}$ & All upazilas ${ }^{+}$ & Lf & GMH 5449 \\
\hline Oryza sativa $\mathrm{L}$. & Dhan & Herb, er; cv & Af & All upazilas & Ed, Lf & MAR 3095 \\
\hline Panicum brevifolium $\mathrm{L}$. & Bashpati ghas & Herb, er; w & Af, Gl, Ml & $\mathrm{Ko}, \mathrm{Ss}$ & $\mathrm{Lf}, \mathrm{Sb}$ & MAR 3095 \\
\hline P. repens $\mathrm{L}$. & Dhani ghas & Herb, er; w & Af, Fl, Gl & All upazilas ${ }^{+}$ & $\mathrm{Lf}, \mathrm{Sb}$ & MAR 3096 \\
\hline $\begin{array}{l}\text { Paspalidium flavidum (Retz.) A. } \\
\text { Camus }\end{array}$ & ghas & Herb, er; w & $\mathrm{Fl}, \mathrm{Gl}, \mathrm{Ml}$ & $\mathrm{Ka}, \mathrm{Sh}, \mathrm{Ss}$ & $\mathrm{Lf}, \mathrm{Sb}$ & MAR 3097 \\
\hline P. conjugatum P.J. Bergius. & Moisshya ghas & Herb, er; w & Gl, Hs, Rs & $\mathrm{Ss}, \mathrm{Sh}^{+}$ & $\mathrm{M}, \mathrm{Sb}$ & MAR 3099 \\
\hline P. distichum $\mathrm{L}$. & Chhotogoicha & Herb, er; w & Ml, Wtl & All upazilas & $\mathrm{Lf}, \mathrm{Sb}$ & MAR 3100 \\
\hline P. scrobiculatum $\mathrm{L}$. & Bishmona ghas & Herb, er; w & $\mathrm{Fl}, \mathrm{Gl}, \mathrm{Rs}$ & As, Ko, Ss & $\mathrm{Lf}, \mathrm{Sb}$ & MAR 3101 \\
\hline P. vaginatum $\mathrm{Sw}$. & Gina ghas & Herb, pr; w & $\mathrm{Gl}, \mathrm{Wtl}$ & $\mathrm{Sh}^{+}$ & $\mathrm{Lf}, \mathrm{Sb}$ & MSR 3336 \\
\hline $\begin{array}{l}\text { Phragmites karka (Retz.) Trin. ex } \\
\text { Steud. }\end{array}$ & Nal khagra & Herb, er;w & $\mathrm{Rb}, \mathrm{Wtl}$ & $\mathrm{Ka}$, & $\mathrm{Hc}, \mathrm{Sb}$ & GMH 5450 \\
\hline $\begin{array}{l}\text { *Porteresia coarctata }(\text { Roxb.) } \\
\text { Tateoka }\end{array}$ & Dhanshi & Herb, er; w & $\mathrm{Rb}, \mathrm{Wtl}$ & $\mathrm{Sh}^{+}$ & $\mathrm{Lf}, \mathrm{Sb}$ & GMH 5451 \\
\hline Saccharum spontaneum $\mathrm{L}$. & Kash & Herb, er; w & $\mathrm{Fl}, \mathrm{Fm}, \mathrm{Rs}$ & $\mathrm{De}, \mathrm{Ka}, \mathrm{Ss}$ & $\mathrm{Sb}, \mathrm{Tm}$ & GMH 5452 \\
\hline Sacciolepis indica (L). A. Chase & Siltatto ghas & Herb, er; w & Ml, Wtl & Ss, Ta & Lf & MAR 3102 \\
\hline Sporobolus indicus (L.) R. Br. & Smut ghas & Herb, er; w & $\mathrm{Fl}, \mathrm{Gl}, \mathrm{Rs}$ & Ko, Ss & $\mathrm{M}, \mathrm{Tm}$ & MAR 3103 \\
\hline Zea mays $\mathrm{L}$. & Bhutta & Herb, er; pl & Af & Ko, Ss & Ed, Lf & MAR 3104 \\
\hline $\begin{array}{l}\text { Zoysia matrella (L.) Merr. } \\
\text { Strelitziaceae Hutch. }\end{array}$ & ghas & Herb, pr; w & $\mathrm{Fl}, \mathrm{Gl}$ & $\mathrm{Sh}^{+}$ & $\mathrm{Lf}, \mathrm{Sb}$ & MSR 3339 \\
\hline $\begin{array}{l}\text { Ravenala madagascariensis Sonn. } \\
\text { Musaceae Juss. }\end{array}$ & Panthopadap & Tree, s; pl & Hs & Ss & $\mathrm{O}$ & KMNI 053 \\
\hline $\begin{array}{l}\text { Musa paradisiaca L. } \\
\text { Typhaceae Juss. }\end{array}$ & Kachkola & Herb, er; w & $\mathrm{Fl}, \mathrm{Hs}, \mathrm{Ml}$ & All upazilas & Fr, Vg & MAR 3105 \\
\hline $\begin{array}{l}\text { Typha elephantina Roxb. } \\
\text { Zingiberaceae Martinov }\end{array}$ & $\mathrm{H}$ & w & $\mathrm{H}$ & S & M & 53 \\
\hline Curcuma longa $\mathrm{L}$. & Halud & Herb, er; cv & $\mathrm{Fl}, \mathrm{Hs}$ & All upazilas & $\mathrm{M}, \mathrm{Sp}$ & KMNI 054 \\
\hline C. zedoaria (Christm.) Rosc. & Sathi & Herb, er; w & $\mathrm{Fl}, \mathrm{Gl}, \mathrm{Rs}$ & As, Ka, Ko, Ta & M, Pf & KMNI 055 \\
\hline Hedychium coronarium J. König & Dolon chapa & Herb, er; cv & Hs, Rs & Ss & $\mathrm{M}, \mathrm{O}$ & KMNI 056 \\
\hline Kaempferia angustifolia Roscoe & Ekangi & Herb, er; w & $\mathrm{Fl}, \mathrm{Wl}$ & De & $\mathrm{M}, \mathrm{O}$ & GMH 5454 \\
\hline Zingiber officinale Roscoe & Ada & Herb, er; cv & $\mathrm{Fl}, \mathrm{Hs}$ & Ka, Ko & $\mathrm{M}, \mathrm{Sp}$ & KMNI 057 \\
\hline $\begin{array}{l}\text { Z. zerumbet (L.) Roscoe ex Sm. } \\
\text { Costaceae Nakai }\end{array}$ & Moha & Herb, er; w & $\mathrm{Fl}, \mathrm{Hs}, \mathrm{Sj}$ & As, Ka, Ko, Ta & $\mathrm{M}, \mathrm{Pf}$ & KMNI 058 \\
\hline $\begin{array}{l}\text { Cheilocostus speciosus (J. Koenig) } \\
\text { C.D. Specht }\end{array}$ & Keomul & Herb, er; w & $\mathrm{Fl}, \mathrm{Wl}$ & Ka, Ko & M & KMNI 059 \\
\hline Cannaceae Juss. & & & & & & \\
\hline Canna indica $\mathrm{L}$. & Kolabati & Herb, er; w & $\mathrm{Fl}, \mathrm{Hs}, \mathrm{Rs}$ & All upazilas & $\mathrm{M}, \mathrm{O}$ & KMNI 060 \\
\hline
\end{tabular}


Table 1 contd.

\begin{tabular}{|c|c|c|c|c|c|c|}
\hline Scientific name & Bangla name & Habit & Habitat & Distribution & Use & RSE \\
\hline \multicolumn{7}{|l|}{ Marantaceae R. Br. } \\
\hline $\begin{array}{l}\text { Schumannianthus dichotomus } \\
\text { (Roxb.) Gagnep. }\end{array}$ & Shital pati & Shrub; w & $\mathrm{Hs}, \mathrm{Wtl}$ & $\mathrm{Ka}$ & $\mathrm{Hc}, \mathrm{M}$ & GMH 5455 \\
\hline \multicolumn{7}{|l|}{ Pontederiaceae Kunth } \\
\hline Eichhornia crassipes (Mart.) Solms & Kachuripana & Herb, ff, w & Wtl & All upazilas & $\mathrm{Gm}, \mathrm{Lf}$ & GMH 5457 \\
\hline Monochoria hastata (L.) Solms & Bara nukha & Herb, er, w & Wtl & Ss, Ta & $\mathrm{Gm}, \mathrm{Vg}$ & MAR 3106 \\
\hline M. vaginalis (Burm. f.) C. Presl & Nukha & Herb, er; w & Wtl & Ss, Ta & $\mathrm{M}, \mathrm{Vg}$ & GMH 5458 \\
\hline \multicolumn{7}{|l|}{ Amaryllidaceae J. St.-Hil. } \\
\hline Allium cepa $\mathrm{L}$. & Piyaj & Herb, er; cv & Af, $\mathrm{Hs}$ & As, Ko, Ta & $\mathrm{M}, \mathrm{Sp}$ & MAR 3109 \\
\hline A. sativum $\mathrm{L}$. & Rashun & Herb, er; cv & Af, $\mathrm{Hs}$ & As, Ko, Ss & $\mathrm{M}, \mathrm{Sp}$ & MAR 3110 \\
\hline Crinum americanum $\mathrm{L}$. & Baro kanur & Herb, er; w & Hs, Wl & As, De, Ko, Ss & $\mathrm{M}, \mathrm{O}$ & MAR 3111 \\
\hline C. asiaticum $\mathrm{L}$. & Shukdarshan & Herb, er; w & Hs, Wl & $\mathrm{De}, \mathrm{Sh}$ & $\mathrm{M}, \mathrm{O}$ & MAR 3112 \\
\hline $\begin{array}{l}\text { Scadoxus multiflorus (Martyn) Raf. } \\
\text { Asparagaceae Juss. }\end{array}$ & Agnigolock & Herb, er; pl & $\mathrm{Fl}, \mathrm{Hs}$ & Sh & $\mathrm{M}, \mathrm{O}$ & MAR 3113 \\
\hline Agave americana $\mathrm{L}$. & Shatabdi udvid & Herb, er; cv & Hs & Ss & $\mathrm{M}, \mathrm{O}$ & MAR 3107 \\
\hline $\begin{array}{l}\text { Cordyline fruticosa (L.) A. Chev. } \\
\text { Hypoxidaceae R. Br. }\end{array}$ & Agnishwar & Herb, er; cv & Hs, Rs & Ss & $\mathrm{M}, \mathrm{O}$ & MAR 3108 \\
\hline \multicolumn{6}{|l|}{ Xanthorrhoeaceae Dumort. } & GMH 5459 \\
\hline \multicolumn{7}{|l|}{ Smilacaceae Vent. } \\
\hline Smilax ovalifolia Roxb. & Kumarika & Herb, cl; w & $\mathrm{Sj}, \mathrm{Wl}$ & As, De, Ka, Ss & M & MAR 3116 \\
\hline \multicolumn{6}{|l|}{ Dioscoreaceae R. Br. } & MAR 3117 \\
\hline Dioscorea alata $\mathrm{L}$. & Chupri alu & Herb, cl; cv & $\mathrm{Hs}, \mathrm{Sj}$ & As, Ka, Sh, Ss & $\mathrm{M}, \mathrm{Vg}$ & GMH 5460 \\
\hline $\begin{array}{l}\text { D. pentaphylla L. } \\
\text { Orchidaceae Juss. }\end{array}$ & Jhum alu & Herb, cl; w & $\mathrm{Sj}, \mathrm{Wl}$ & Ko, Ss & $\mathrm{M}, \mathrm{Vg}$ & GMH 5461 \\
\hline $\begin{array}{l}\text { *Luisia brachystachys (Lindl.) } \\
\text { Blume }\end{array}$ & Borolucia & Herb, ep; w & Op & $\mathrm{Sh}^{+}$ & $\mathrm{M}, \mathrm{O}$ & MSR 3333 \\
\hline *L. tristis (G.Forst.) Hook. f. & Lucia & Herb, ep; w & Op & $\mathrm{Sh}^{+}$ & $\mathrm{O}, \mathrm{Pf}$ & GMH 5462 \\
\hline $\begin{array}{l}\text { Geodorum densiflorum (Lam.) } \\
\text { Schltr. }\end{array}$ & Sankhamul & Herb, er; w & $\mathrm{Sj}, \mathrm{Wl}$ & $\mathrm{De}, \mathrm{Sh}$ & M, O & GMH 5463 \\
\hline $\begin{array}{l}\text { Vanda tessellata (Roxb.) Hook. ex } \\
\text { G. Don. }\end{array}$ & Rasna & Herb, ep; w & Op & Ss & $\mathrm{M}, \mathrm{O}$ & GMH 5464 \\
\hline *Zeuxine strateumatica $(\mathrm{L}$.) Schltr. & Setguli & Herb, er; w & $\mathrm{Gl}, \mathrm{Ml}$ & $\mathrm{Sh}^{+}$ & $\mathrm{O}$ & GMH 5465 \\
\hline
\end{tabular}

Notes: Habit: cl- climber, cr- creeper, cv- cultivated, em- emergent, ep- epiphyte, er- erect, ff- free floating, fr- floating with rooted, l- large, li- liana, lp- lithophyte, m- medium, pl- planted pr- prostrate, ps- parasite, s-small, sc- scandant, smsubmerged, vi- vine, w- wild. Habitat: Af- Agri-field, Fl- Fallow land, Fm- Forest margin, Gl- Grassland, Hs- Homestead, Ml- Marginal land, Obw- On brick wall, Op- On plant, Rb- River bank, Rs- Roadside, Sj- Scrub jungle, Wl- Woodland, Wtl- Wetland. Distribution: As- Ashasuni upazila, De- Debhata upazila, Ka- Kaligonj upazila, Ko- Kolarowa upazila, ShShaymnagar upazila, Ss- Satkhira sadar upazila, Ta- Tala upazila. + (plus mark)- distribution in Sundarban mangrove forest of Shaymnagar upazila; * (asterisk mark) - Species recorded only from the Sundarban part of Satkhira district. Uses: Ap- aquarium plant, Co- Cosmetics, Dy- Dye yielding, Ed- Edible, Fb- Fibre, Ff- Fish feed, Fp- Fish poison, Fr- Fruit, FwFuel wood, Gm- Green manure, Gu- Gum, Hc- Handicrafts, He- Hedge, Hp- Honey plant, Ju- Juice, Lf- Livestock feed, M- Medicine, O- Ornamental, Oy- Oil yielding, Pf- Perfume, Pp- Paper pulp, Pu- Pulse, Sb- Soil binder, Sp- Spice, TTimber, Tm- Theaching material, Vg- Vegetable, Wp- Water purify; $\boldsymbol{R S E}$ (Representative Specimen Examined): GMHGazi Mosharof Hossain, KMNI- Khandaker Mohammad Noor Islam, MAR- Md. Abdur Rahim, MSR- Mohammad Sayedur Rahman, SAK- Saleh Ahammad Khan. 
Total 147 plant species including 113 species of dicotyledons, 31 species of monocotyledons and three species of Pteridophytes were found in all upazilas, but no species of Gymnosperms was commonly found in all upazilas. Only 71, 44, 14, 12, six and four species were exclusively found in Shyamnagar, Satkhira Sadar, Kaligonj, Debhata, Kolaroa and Tala upazila, respectively and no species was exclusively distributed in Assashuni upazila. In this district, the plant species were found in diverse habitats, however, most of the species were well-adapted in fallow lands and roadsides, which were followed by, woodlands, homesteads, agricultural fields, scrub jungles, grass lands, wetlands, marginal lands (including forest margins and river banks). Some species were also found on few tree species and on brick walls (Fig. 3).

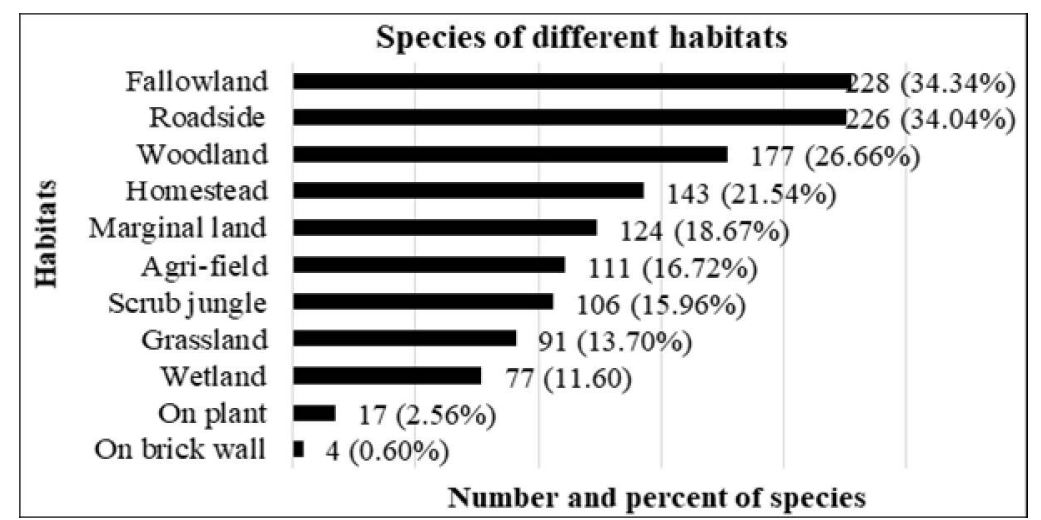

Fig. 3. Distribution of plant species in different habitats of Satkhira district.

All species recorded from Satkhira district are economically or ecologically useful and most of these species have more than one uses. Most of the species $(476 ; 71.69 \%)$ are useful as medicine, which are followed by $112(16.87 \%)$ species of ornamentals, $73(10.99 \%)$ of vegetables, $50(7.53 \%)$ of fruits and livestock feed each, $38(5.72 \%)$ of timbers, $33(4.97 \%)$ of fibres and 26 (3.92\%) of fuel woods. Additionally, $19(2.86 \%)$ species have been distinguished as useful in producing green manure, $17(2.56 \%)$ in dye yielding, $14(2.11 \%)$ in handicrafts making, 12 $(1.81 \%)$ as hedge, $12(1.81 \%)$ as thatching material, $11(1.66 \%)$ as spices and $10(1.51 \%)$ as oil yielding. Few species have been recognized as useful as pulse, water purifying, perfume, cosmetics, edible, fish feed, paper pulp, fish poison, gum, honey plant and juice plant.

In the study area, 17 species, viz. Acanthus volubilis (Acanthaceae), Andrographis paniculata (Acanthaceae), Baliospermum solanifolium (Euphorbiaceae), Barringtonia acutangula (Lecythidaceae), Casearia tomentosa (Salicaceae), Cerbera odollam (Apocynaceae), Diospyros malabarica (Ebenaceae), Dodonaea viscosa (Sapindaceae), Dolichandrone spathacea (Bignoniaceae), Geodorum densiflorum (Orchidaceae), Kaempferia angustifolia (Zingiberaceae), Luisia brachystachys (Orchidaceae), Parkinsonia aculeata (Caesalpiniaceae), Pavetta indica (Rubiaceae), Rauvolfia serpentina (Apocynaceae), Scadoxus multiflorus (Amaryllidaceae) and Zeuxine strateumatica (Orchidaceae) were found as rare with small population, poor regeneration and restricted distribution. Among these species, A. paniculata and R. serpentina are listed as threatened species for Bangladesh (Khan et al., 2001).

The taxonomic enumeration of Angiosperm species reported by this study from Satkhira district covering $3,817.29 \mathrm{~km}^{2}$ area, is nearly similar to that of Narsingdi district composed of $3360.59 \mathrm{~km}^{2}$ area (Khanam et al., 2020; Khanam and Khan, 2020), lower than those reported from Patuakhali district of $3221.31 \mathrm{~km}^{2}$ area (Sultana, 2012) and Gazipur district of $1806.36 \mathrm{~km}^{2}$ area 
(Tabassum, 2015) and higher than that recorded from Rajshahi district situated on $2407.01 \mathrm{~km}^{2}$ area (Rahman, 2013).

On the other hand, the taxonomic account of Angiosperm species found in six of the seven upazilas of Satkhira district, viz. Satkhira Sadar, Kaligonj, Shaymnagar, Debhata, Kolarowa and Tala, are higher than those previously reported from different upazilas of some districts of the country, viz. Ramgarh upazila of Khagrachhari district (Islam et al., 2009), Dhamrai upazila of Dhaka district (Rahman et al., 2012), Sadar upazila of Munshiganj district (Rahman et al., 2013), Manikgonj Sadar upazila of Manikgonj district (Sarker et al., 2013), Sadar upazila of Naogaon district (Nahar and Rahman, 2016), Mahadebpur upazila of Naogaon district (Rahman and Kona, 2016), Sreenagar upazila of Munshigang district (Mahmudah et al., 2017), Ishwardi of Pabna district (Roy and Rahman, 2018), and Gafargaon upazila of Mymensingh district (Rahman et al., 2019) (Fig. 4). In contrast, this enumeration of Angiosperm species is lower than those reported from Belabo and Shibpur upazilas and nearly similar to those reported from Monohordi and Sadar upazila of Narsingdi district (Khanam et al., 2020; Khanam and Khan, 2020) (Fig. 4).

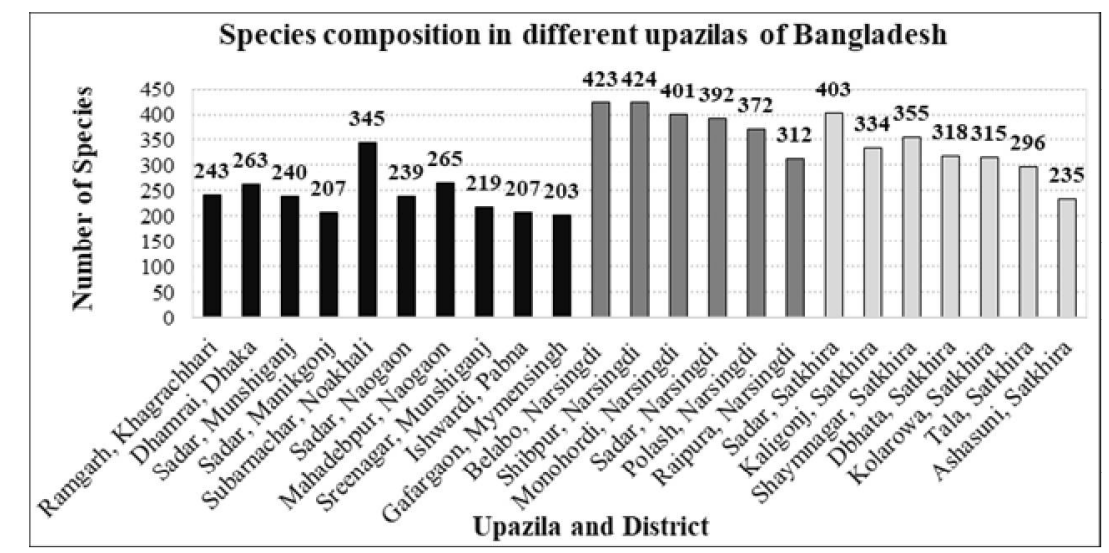

Fig. 4. Angiosperms species composition in different upazilas of Satkhira district in comparison to previous records from different upazilas of some districts of Bangladesh.

Satkhira district is a vulnerable coastal area of Bangladesh due to both natural disasters and anthropogenic causes (Rahman and Ferdous, 2017). Frequent natural disasters, increasing salinity intrusion in both soil and water, invasion of some exotic species (Acacia auriculiformis, Eucalyptus camaldulensis, Prosopis juliflora), poor regeneration, waterlogging and lack of proper management programs etc. are the critical natural problems and threats for its habitat- and ecosystem degradation, the consequences of which are generating colossal negative effects on the local flora and vegetation in this district (Kabir and Eva, 2014). Satkhira is one of the three districts of Bangladesh that accommodate around $80 \%$ of total shrimp production (Chowdhury and Muniruzzaman, 2003; Karim, 2003). But the widespread and increasing shrimp farming in this district is now a major anthropogenic threat that is likely to bring severe damages to the local environment and ecosystems, and ultimately to the local flora and vegetation (Kabir and Eva, 2014). However, the flora of the study area is still rich and withstanding through facing frequent natural disasters and severe human interferences, though some of its areas are almost bare.

The taxonomic information provided by this study on the vascular plants of Satkhira districts, might serve as an important guiding database to track the trend of changes in the floristic composition, plant species diversity and vegetation in course of time, especially due to natural and 
anthropogenic threats, contribute in undertaking appropriate biodiversity conservation initiatives and plant resource-based socio-economic development, and help in estimating the impacts of climate change in this area. This study highly recommends for regular inventories, monitoring and problem-specific research programs on the flora and plant diversity of this district, conducting intensive research for finding the effective wind and saline tolerant plant species, accomplishment of adequate plantation with appropriate species in the marginal lands of coastal- and river bank areas and implementation of adequate measures for conservation of the threatened and depleting plant species of this district.

\section{Acknowledgements}

The authors express their sincere thanks to Grant for Advanced Research in Education (GARE), BANBEIS, for funding this study. The authors are grateful to all people who were directly or indirectly involved in this study. The authors are thankful to the Chief Editor and the Reviewers of the Journal for their critical review of the manuscript.

\section{References}

Ahmed, Z.U., Begum, Z.N.T., Hassan, M.A., Khondker, M., Kabir, S.M.H., Ahmad, M., Ahmed, A.T.A., Rahman, A.K.A. and Haque, E.U. (Eds). 2008-2009. Encyclopedia of Flora and Fauna of Bangladesh. Vols. 6-8 \& 12. Asiatic Society of Bangladesh, Dhaka.

Ahmed, Z.U., Hassan, M.A., Begum, Z.N.T., Khondker, M., Kabir, S.M.H., Ahmad, M., Ahmed, A.T.A. (Eds). 2009. Encyclopedia of Flora and Fauna of Bangladesh. Vols. 9-10. Asiatic Society of Bangladesh, Dhaka.

Arefin, M.K., Rahman, M.M., Uddin, M.Z. and Hassan, M.A. 2011. Angiosperm flora of Satchari National Park, Habiganj, Bangladesh. Bangladesh J. Plant Taxon. 18(2): 117-140.

Bridson, D.M. and Forman, F. 1989. In: Bridson, D.M. and Forman, F. (Eds). The Herbarium Handbook. Royal Botanic Gardens, Kew, 214 pp.

Choudhury, A.M. 2002. Managing natural disasters in Bangladesh. In: The Dhaka Meet on Sustainable Development in Bangladesh: Achievements, Opportunities and Challenges at Rio+10. Bangladesh Unnayan Parishad.

Chowdhury, M.B.R. and Muniruzzaman, M. 2003. Shrimp Diseases and Its' Consequences on the Coastal Shrimp Farming in Bangladesh. In: Wahab, M.A. (Ed). Environmenal and Socioeconomic Impacts of Shrimp Farming in Bangladesh. Dhaka, pp. 39-48.

Chowdhury, M.S.U. 1996. Bangladesh: country report to the FAO international technical conference on plant genetic resources, Leipzig, pp. 1-112.

Cronquist, A. 1981. An Integrated System of Classification of Flowering Plants. Columbia University Press, New York, pp. 1-1262.

Ghani, A. 1998. Medicinal Plants of Bangladesh with Chemical Constituents and Uses. Asiatic Society of Bangladesh, pp. 1-467.

Haque, A.K.M.K., Khan, S.A., Uddin, S.N. and Shetu, S.S. 2018. An annotated checklist of the Angiospermic flora of Rajkandi Reserve Forest of Moulvibazar. Bangladesh. Bangladesh J. Plant Taxon. 25(2): 187-207.

Hooker, J.D. 1872-1897. The Flora of British India. Vols. 1-7. L. Reeve \& Co., Ashford, Kent, UK.

Huq, A.M. 2019. Plant names of Bangladesh. Ashrafia Boi Ghor, 36, Banglabazar, Dhaka, pp. 1-578.

Islam, M.R., Uddin, M.Z. and Hassan, M.A. 2009. An Assessment of the Angiospermic Flora of Ramgarh Upazila of Khagrachhari District. Bangladesh. Bangladesh J. Plant Taxon. 16(2): 115-140.

Kabir, M.H. and Eva, I.J. 2014. Ecological Consequences of Shrimp Farming in Southwestern Satkhira District of Bangladesh. Austin J Earth Sci. 1(1): 7. 
Karim, M.R. 2003. Present Status and Strategied for Future Development of Shrimp Farming in Bangladesh, In: Wahab, M. (Ed). Environmental and Socioeconomic Impacts of Shrimp Farming in Bangladesh, Dhaka, pp. 1-8.

Khan, M.S. 1977. Onagraceae. In: Khan, M.S. (Ed). Flora of Bangladesh. Fasc. 6: 1-10. Bangladesh National Herbarium, BARC, Dhaka.

Khan, M.S. and Huq, A.M. 2001. The vascular flora of Chunati wildlife sanctuary in south Chittagong. Bangladesh J. Plant Taxon. 8(1): 47-64.

Khan, M.S., Rahman, M.M. and Ali, M.A. (Eds). 2001. Red Data Book of Vascular Plants of Bangladesh. Vol. 1. Bangladesh National Herbarium, Dhaka, Bangladesh, pp. 1-179.

Khanam, R. and Khan, S.A. 2020. Angiosperms in Narsingdi District of Bangladesh: Class Liliopsida. Bangladesh J. Plant Taxon. 27(2): 391-405.

Khanam, R., Khan, S.A. and Rahim, M.A. 2020. Angiosperms in Narsingdi District of Bangladesh: class Magnoliopsida. Bangladesh J. Plant Taxon. 27(1): 153-271.

Kramer, K.U. and Green, P.S. 1990. Pteridophytes and gymnosperms. In: Kubitzki K. The families and genera of vascular plants. Vol. 1. Springer, Berlin.

Mahmudah, Z., Islam, M.M, Haque, T. and Uddin, M.Z. 2017. Taxonomic Enumeration of Angiosperm Flora of Sreenagar Upazila, Munshigang, Dhaka, Bangladesh. J. Asiat. Soc. Bangladesh, Sci. 43(2): 161-172.

Nahar, J. and Rahman, A.H.M.M. 2016. Study of Angiosperm Plant Species at Sadar Upazila of Naogaon District, Bangladesh. Discovery 52(250): 1963-1978.

Ostertag, R., Inman-Narahari, F., Cordell, S., Giardina, C.P. and Sack, L. 2014. Forest structure in lowdiversity tropical forests: A study of Hawaiian wet and dry forests. PLoS ONE9: e103268. Doi:10.1371/journal.pone.0103268.

Pichi, S.R.E.G. 1977. Tentamen pteridophytorum genera in taxonomicum ordinem redigendi. Webbia 31: 313-512.

Population and Housing Census, 2015. Zila Report: Satkhira (2011). Statistics and Informatics Division, Bangladesh Bureau of Statistics. Ministry of Planning, Government of the People's Republic of Bangladesh, pp. 1-325.

Prain, D. 1903. Bengal Plants. Vols. 1 \& 2. Reprint 1963. Botanical Survey of India, Calcutta.

Rahman, A.H.M.M. and Kona, S. 2016. Angiosperm Systematics of Mahadebpur Upazila of Naogaon, Bangladesh. Lambert Academic Puublishing, https://www.researchgate.net/publication/296705290.

Rahman, M.A. 2017. Plant Diversity in Hazarikhil Wildlife Sanctuary of Chittagong and its Conservation Management. J. biodivers. Conserv. Bioresour. Manag. 3(2): 43-56.

Rahman, M.O., Antara, R.T., Begum, M. and Hassan, M.A. 2012. Floristic diversity of Dhamrai Upazila of Dhaka with emphasis on medicinal plants. Bangladesh J. Bot. 41(1): 71-85.

Rahman, M.O., Begum, M. and Ullah, M.W. 2013. Angiosperm Flora of Sadar Upazila of Munshiganj District, Bangladesh. Bangladesh J. Plant Taxon. 20(2): 213-231.

Rahman, M.O., Sayma, N.J. and Begum, M. 2019. Angiospermic flora of Gafargaon Upazila of Mymensingh District focusing on medicinally important species. Bangladesh J. Plant Taxon. 26(2): 269-283.

Rahman, M.S., Hossain, G.M., Khan, S.A. and Uddin, S.N. 2015. An Annotated Checklist of the Vascular Plants of Sundarban Mangrove forest of Bangladesh. Bangladesh J. Plant Taxon. 22(1): 17-41.

Rahman, M.T. and Ferdous, J. 2017. Detection of Environmental Degradation of Satkhira District, Bangladesh through Remote Sensing Indices. DOI: 10.1007/978-981-10-8016-6_74.

Rashid, S.H. and Mia, M.M.K. 2001. Angiospermic flora of Madhupur National Park, Tangail, Bangladesh. Bangladesh J. Plant Taxon. 8(2): 63-82.

Roy, G.K. and Khan, S.A. 2020. Preliminary taxonomic study on homestead flora of four districts of Bangladesh: Magnoliopsida. Bangladesh Journal of Plant Taxon. 27(1): 37-65.

Roy, T.R. and Rahman, A.H.M.M. 2018. Inventory of Angiosperm Diversity in Iswardi Proshova of Pabna District, Bangladesh. Analysis 14: 09-22. 
Sarker, K., Islam, M.R., Uddin, M.Z., and Hassan, M.A. 2013. Angiosperm Flora of Manikgonj Sadar Upazila, Bangladesh. J. Asiat. Soc. Bangladesh, Sci. 39(2): 147-166.

Schaminée, J.H.J., Janssen, J.A.M., Hennekens, S.M. and Ozinga, W.A. 2011. Large vegetation databases and information systems: new instruments for ecological research, nature conservation, and policy making. Plant Biosyst 145: 85-90.

Sharrock, S. 2012. Global Strategy for Plant Conservation: A guide to the GSPC. All the targets, objectives and facts. Richmond, UK (Botanic Gardens Conservation International, 2012).

Shetu, S.S., Khan, S.A. and Uddin, S.N. 2018. Checklist of Angiosperms extant in Mirpur area of Dhaka City, Jahangirnagar University J. Biol. Sci. 7(2): 47-64.

Siddiqui, K.U., Islam, M.A., Ahmed, Z.U., Begum, Z.N.T., Hassan, M.A., Khondker, M., Rahman, M.M., Kabir, S.M.H., Ahmad, M., Ahmed, A.T.A., Rahman, A.K.T. and Haque, E.U. (Eds). 2007. Encyclopedia of Flora and Fauna of Bangladesh. Vols. 5 \& 11. Asiatic Society of Bangladesh, Dhaka.

Singh, H.B. and Subramaniam, B. 2008. Field Manual on Herbarium Techniques. National Institute of Science Communication and Information Resources, pp. 1-297.

Sultana, M. 2012. Taxonomic and ethnobatanical studies on the angiospermic flora of Patuakhali District in Bangladesh. Ph.D. Thesis (Unpublished). Department of Botany, University of Dhaka, pp. 1-565.

Tabassum, R. 2015. Angiospermic flora of Gazipur District, Bangladesh. Doctoral Dissertation. Department of Botany, University of Dhaka, pp. 1-707.

The Plant List, 2013. The Plant List, a working list of all plant species. Version $1.1<\mathrm{http}: / /$ www.t heplantlist. org/>. Accessed on 15 October 2018.

TROPICOS, 2017. Tropicos.org. <www.tropicos.org>. Missouri Botanical Garden, Saint Louis, Missouri, USA. Accessed on 14 July 2019.

Uddin, M.Z., Hassan, M.A. and Khan, M.S. 2003. An annotated checklist of Angiospermic Flora of RemaKalenga Wildlife Sanctuary (Habiganj) in Bangladesh IIa: Magnoliopsida (Dicots). Bangladesh J. Plant Taxon. 10(1): 79-94.

Uddin, S.N. and Hassan, M.A. 2018. Vascular Flora of Chittagong and the Chittagong Hill Tracts: Vols.1-3. Bangladesh National Herbarium, Zoo Road, Mirpur 1, Dhaka 1216.

Uddin, S.N., Khan, M.S., Hassan, M.A. and Alam, M.K. 1998. An annotated checklist of angiospermic flora of Sitapahar at Kaptai in Bangladesh. Bangladesh J. Plant Taxon. 5(1): 13-46.

Van Valkenburg, J.L.C.H. and Bunyapraphatsara. N. (Eds). 2002. Plant Resources of South-East Asia. No. 12(2). Medicinal and Poisonous Plants 2. Prosea Foundation, Bogor, Indonesia, 782 pp.

WCMC, 1992. World Conservation Monitoring Centre. Global Biodiversity: Status of Earth's Living Resources. Chapman and Hall, London, UK.

World Bank, 2005. Natural disaster Hotspots: A global risk analysis (Disaster Risk Management Series, No. 5). Washington, DC.

Wu, Z.Y. and Raven, P.H. (Eds). 1994-2001. Flora of China. Vols. 8, 15-18, and 24. Missouri Botanical Garden Press, St. Louis, USA.

Wu, Z.Y., Raven, P.H. and Hong, D.Y. (Eds). 1999-2013. Flora of China. Vols. 2-7, 9-14, 19-23, and 25. Missouri Botanical Garden Press, St. Louis. 